\title{
AP-Net: An Atomic-Pairwise Neural Network for Smooth and Transferable Interaction Potentials
}

\author{
Zachary Glick, Derek Metcalf, Alexios Koutsoukas, Steven Spronk, Daniel Cheney, David Sherrill
}

Submitted date: 05/05/2020 - Posted date: 06/05/2020

Licence: CC BY-NC-ND 4.0

Citation information: Glick, Zachary; Metcalf, Derek; Koutsoukas, Alexios; Spronk, Steven; Cheney, Daniel; Sherrill, David (2020): AP-Net: An Atomic-Pairwise Neural Network for Smooth and Transferable Interaction Potentials. ChemRxiv. Preprint. https://doi.org/10.26434/chemrxiv.12246020.v1

Intermolecular interactions are critical to many chemical phenomena, but their accurate computation using ab initio methods is often limited by computational cost. The recent emergence of machine learning $(\mathrm{ML})$ potentials may be a promising alternative. Useful ML models should not only estimate accurate interaction energies, but also predict smooth and asymptotically correct potential energy surfaces. However, existing ML models are not guaranteed to obey these constraints. Indeed, systemic deficiencies are apparent in the predictions of our previous hydrogen-bond model as well as the popular ANI-1X model, which we attribute to the use of an atomic energy partition. As a solution, we propose an alternative atomic-pairwise framework specifically for intermolecular ML potentials, and we introduce AP-Net-a neural network model for interaction energies. The AP-Net model is developed using this physically motivated atomic-pairwise paradigm and also exploits the interpretability of symmetry adapted perturbation theory (SAPT). We show that in contrast to other models, AP-Net produces smooth, physically meaningful intermolecular potentials exhibiting correct asymptotic behavior. Initially trained on only a limited number of mostly hydrogen-bonded dimers, AP-Net makes accurate predictions across the chemically diverse S66x8 dataset, demonstrating significant transferability. On a test set including experimental hydrogen-bonded dimers, AP-Net predicts total interaction energies with a mean absolute error of $0.37 \mathrm{kcal} \mathrm{mol}-1$, reducing errors by a factor of 2-5 across SAPT components from previous neural network potentials. The pairwise interaction energies of the model are physically interpretable, and an investigation of predicted electrostatic energies suggests that the model 'learns' the physics of hydrogen-bonded interactions.

File list (2) 


\title{
AP-Net: An atomic-pairwise neural network for smooth and transferable interaction potentials
}

\author{
Zachary L. Glick, ${ }^{1}$ Derek P. Metcalf, ${ }^{1}$ Alexios Koutsoukas, ${ }^{2}$ Steven A. Spronk, ${ }^{2}$ Daniel L. Cheney, ${ }^{2}$ and \\ C. David Sherrill ${ }^{1}$ \\ ${ }^{1)}$ Center for Computational Molecular Science and Technology, School of Chemistry and Biochemistry, \\ and School of Computational Science and Engineering, Georgia Institute of Technology, Atlanta, Georgia 30332-0400, \\ $U S A$ \\ ${ }^{2)}$ Molecular Structure and Design, Bristol Myers Squibb Company, P. O. Box 5400, Princeton, \\ NJ 08543
}

Intermolecular interactions are critical to many chemical phenomena, but their accurate computation using $a b$ initio methods is often limited by computational cost. The recent emergence of machine learning (ML) potentials may be a promising alternative. Useful ML models should not only estimate accurate interaction energies, but also predict smooth and asymptotically correct potential energy surfaces. However, existing ML models are not guaranteed to obey these constraints. Indeed, systemic deficiencies are apparent in the predictions of our previous hydrogen-bond model as well as the popular ANI-1X model, which we attribute to the use of an atomic energy partition. As a solution, we propose an alternative atomic-pairwise framework specifically for intermolecular ML potentials, and we introduce AP-Net - a neural network model for interaction energies. The AP-Net model is developed using this physically motivated atomic-pairwise paradigm and also exploits the interpretability of symmetry adapted perturbation theory (SAPT). We show that in contrast to other models, AP-Net produces smooth, physically meaningful intermolecular potentials exhibiting correct asymptotic behavior. Initially trained on only a limited number of mostly hydrogen-bonded dimers, AP-Net makes accurate predictions across the chemically diverse S66x8 dataset, demonstrating significant transferability. On a test set including experimental hydrogen-bonded dimers, AP-Net predicts total interaction energies with a mean absolute error of $0.37 \mathrm{kcal} \mathrm{mol}^{-1}$, reducing errors by a factor of $2-5$ across SAPT components from previous neural network potentials. The pairwise interaction energies of the model are physically interpretable, and an investigation of predicted electrostatic energies suggests that the model 'learns' the physics of hydrogen-bonded interactions.

\section{INTRODUCTION}

Recent advances in the field of machine learning (ML) offer an exciting new perspective on the perpetual costaccuracy trade-off of quantum chemistry. ${ }^{1 / 2}$ Models like neural networks (NNs), which are flexible universal function approximators, can be used to predict a variety of chemical properties. For a target molecular system, a parameterized (or trained) model can estimate a property in a small fraction of the time needed to compute it with quantum methods. These models come with some caveats, however. The accuracy of a prediction is highly dependent on the amount of data used to train the model and the similarity of the training data to the molecule to be predicted on. Also, it is nontrivial to design and train an ML model. Specific choices made in the architecture of a neural network, the choice of features, hyperparameters, etc. can have a large impact on the model's accuracy and transferability.

Over the past few years, ML models have been developed for seemingly every chemical property that can be computed with an ab initio method. The chemistry community has investigated electrostatic multipoles 3 , rate constants $^{4}$, chemical shifts ${ }^{5}$, etc. However, a significant amount of attention has been dedicated to ML potentials (i.e. energy prediction) $\left[\frac{6][18}{[1}\right.$ These ML potentials possess one commonality, which is that their development and application have emphasized covalently bound systems and accurate total energy predictions. Less attention has been paid to noncovalent interactions (NCIs) and interaction energies, which are of fundamental importance to drug binding, liquid structure, biomolecular structure, molecular crystals, etc. In many of these applications, obtaining accurate interaction energies is a more important goal than total energies. This is not to say that existing ML potentials totally neglect NCIs. The ANI-1X model, for example, was trained using a dataset containing many molecular dimers ${ }^{[14}$ Also, accurate total energy predictions can be used to obtain accurate intermolecular energy predictions. For a dimer, the interaction energy $\left(\Delta E_{\text {int }}\right)$ is defined as:

$$
\Delta E_{\text {int }}=E_{A B}-E_{A}-E_{B},
$$

where $E_{A B}, E_{A}$, and $E_{B}$ are total energies of the dimer and two monomers. For any ML potential, dimer interaction energies can be evaluated with this so-called 'supermolecular' approach. One challenge for NCIs is that to obtain accurate interaction energy, one needs good cancellation of errors in all three component energies, $E_{A B}$, $E_{A}$, and $E_{B}$.

Alternatively, more accurate models of interaction energies might estimate $\Delta E_{\text {int }}$ directly. Creating such an intermolecular ML potential poses some unique challenges, as many standard representations of molecular systems for ML are not necessarily applicable, and special care must be taken in generating useful training data. These and other concerns are discussed in our recent 
pilot study of hydrogen-bonding interactions, which to our knowledge is the first purely ML intermolecular potential designed to work on an entire class of chemical systems ${ }^{19}$ In that work, neural networks were trained to predict symmetry adapted perturbation theory (SAPT) interaction energies, resulting in a model which we refer to here as SAPT-ML. The SAPT-ML intermolecular potential was designed in the same spirit as many of the popular total potentials, including the use of an atomic energy partition, discussed in more detail in Section IIB. On a dataset of crystallographic and artificially constructed hydrogen bonding dimers, SAPT-ML predicted interaction energies with a mean absolute error of $1.2 \mathrm{kcal} \mathrm{mol}^{-1}$, approaching quantitative accuracy, while using a relatively small training dataset for deep learning tasks.

The primary evaluation metric for any potential, SAPT-ML included, is generally a summary of the error distribution such as the mean absolute error, max absolute error, etc. preferably computed for a large and representative test dataset. A useful ML potential will make predictions with small errors, but these summary statistics alone are not sufficient criteria for usefulness. Of arguably equal importance is the smoothness of the predicted potential energy surface (PES). Jagged PESs yield inaccurate forces, which is a particularly problematic concern for molecular dynamics simulations, a target application of ML potentials. Smoothness is also a requirement for geometry minimization and transition state searches. For an intermolecular PES, an ML potential should adhere to additional asymptotic constraints. The predicted interaction energy should be approximately zero at large separations and strongly repulsive at near separations. The predicted PES should not only be locally smooth, but also exhibit minima with approximately the same energies and locations as the true PES.

The importance of smoothness for ML potentials has been acknowledged by ML approaches that use energy gradients in the training procedure, 200 but to our knowledge global PES smoothness has not been studied in detail, particularly in the context of intermolecular interactions. In the pilot hydrogen-bonding study, we did not explicitly examine smoothness nor asymptotic convergence of the SAPT-ML model. Here, we re-examine our SAPT-ML potential and also investigate the popular ANI-1X potential. We find that both models produce intermolecular PESs with unphysical irregularities. This appears to be a fundamental weakness of atomic neural network potentials, which predict an atomic partition of the target property. To overcome this deficiency we propose an alternative atomic-pairwise interaction paradigm, and we introduce AP-Net, a corresponding atomic-pairwise neural network intermolecular potential. To illustrate the advantage of this new approach, we test AP-Net on the same hydrogen-bonding task as SAPT$\mathrm{ML}$ and report up to a 5 -fold reduction in errors across the SAPT interaction energy components. The AP-
Net model, trained only on a modest number of mostly hydrogen-bonded dimers, is also tested on the chemically diverse S66x8 benchmark dataset ${ }^{211}$ This atomic-pairwise models exhibits surprising transferability, making reasonable estimates of intermolecular PESs dominated by $\pi-\pi$ stacking and dispersion interactions for systems where ANI-1X generates incorrect potentials. Overall, we observe that AP-Net uniquely displays correct asymptotic behavior and makes smooth predictions along intermolecular coordinates, both necessary aspects of intermolecular potentials. Lastly, we examine the individual atomicpair energies predicted by AP-Net and find good agreement with chemical intuition. This suggests that AP-Net predicts interaction energies by 'learning' some physically meaningful chemical representation.

\section{METHODOLOGY}

\section{A. Symmetry Adapted Perturbation Theory}

In order to train an intermolecular ML potential, reference interaction energies (or labels) are needed from some quantum chemistry calculation. In principle, any wavefunction or density functional theory (DFT) method could be used in conjunction with Equation 1 to obtain supermolecular reference interaction energies. An alternative theoretical approach for computing the interaction energy is symmetry adapted perturbation the-

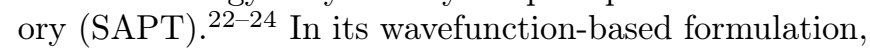
SAPT accounts for the interaction between two monomer Hartree-Fock wavefunctions through a triple perturbation series in monomer A correlation, monomer B correlation, and intermonomer interaction. SAPT has a few advantages over the supermolecular approach. Firstly, SAPT is formally correct in the limit of the perturbation series, and it recovers the full configuration interaction solution to the time-independent Schrödinger equation. However, a major appeal of SAPT is the accuracy of early truncations of the series. One of the most popular and economical SAPT methods, $0^{\text {th }}$ order intramonomer correlation and $2^{\text {nd }}$ order intermonomer interaction, is commonly referred to as SAPT0. Combined with an appropriate jun-cc-pVDZ basis set, SAPT0 has been shown to be surprisingly accurate, in part due to fortuitous error cancellation ${ }^{25}$ At only $\mathcal{O}\left(N^{5}\right)$ in cost, SAPT0 is is affordable enough to probe interactions in systems with hundreds of atoms, large enough to be of biological interest. ${ }^{26}$

The most useful feature of SAPT for an ML potential, however, is the resulting physically meaningful decomposition of the interaction energy. The individual terms of the perturbation series correspond to standard interpretations of intermolecular interactions: interactions between permanent charge distributions of opposite monomers (electrostatics, $\left.E_{\text {elst }}\right)$, induction or polarization of a charge distribution on one monomer by a charge distribution on the other (induction, $E_{i n d}$ ), simultaneous correlation between charge distributions on opposite 
monomers (dispersion, $E_{\text {disp }}$ ), and finally fermionic Pauli exchange between electrons on opposite monomers (exchange, $\left.E_{\text {exch }}\right)$ :

$$
\Delta E_{\text {int }} \approx E_{\text {SAPT0 }} \equiv E_{\text {elst }}+E_{\text {exch }}+E_{\text {ind }}+E_{\text {disp }} .
$$

These components are often exploited in modern force field development, where separate physically-motivated functional forms are developed and parameterized for each term. .274 An analogous approach can be taken in an intermolecular ML potential by structuring a model to predict these individual components, therefore allowing the model to exploit the interpretability of SAPT. Detailed equations for the exact specification of individual SAPT0 components and their efficient implementation through density-fitting techniques are presented in Refs 35 and 36 .

\section{B. Pairwise Energy Partition}

Nearly every published ML potential follows the same general formulation. For a given molecular system, there exist many arbitrary partitions of the total energy $(E)$ into atomic energies $\left(E_{i}\right)$ :

$$
E=\sum_{i} E_{i} .
$$

It is assumed that a partition exists such that $E_{i}$ is a learnable and transferable function of the local environment around atom $i$. Regression models such as neural networks are used to predict $E_{i}$ for each atom. No $a b$ initio $E_{i}$ labels exist, as atomic partitions of the energy are not generally computable with quantum mechanical methods. Instead, the sum of predicted $E_{i}$ 's is constrained to match $E$, which in most ML frameworks is easily specified. Thus, the predicted total energy is estimated through a learned atomic energy partition. The various ML approaches primarily differ in how the regression model is parameterized to predict the partitioned energy $E_{i}$ of each atom $i$.

The atomic nature of this type of potential has an important consequence. Because the regression is performed at the atomic level, models are transferable to many different molecules, provided that the local environments of atoms in the molecules are similar. For example, an atomic potential trained on conformations of butane and hexane should provide reasonable estimations for the PES of a pentane molecule. The transferability of atomistic models of this kind can be contrasted with traditional system-specific potentials, which must be created and parameterized separately for each molecular system of interest.

A weakness of atomic ML potentials is their poor performance in capturing long-range interactions. $\frac{10113}{10}$ This is because the enforced locality of the atomic partition is at odds with the long-range nature of NCIs. Most ML potentials limit an atom's environment to include neighboring atoms within some distance cutoff that can be as short as $5 \AA$. This is insufficient in capturing small but chemically important long-range electrostatics, and to a lesser degree, van der Waals effects. If one chose to use spatially large atomic environments, the transferability and computational cost of the resulting ML model would suffer. To address this issue, some machine-learning potentials simply add a classical force-field model (like Grimme's D3 dispersion correction) to their predictions in order to describe distant interactions ${ }^{9113}$. Other models are parameterized to predict atomic charges, which are used to evaluate a separate long-range electrostatic energy 17

In an attempt to explore the modeling of NCIs with flexible neural network models, we recently adapted the atomic partitioning approach to the explicit prediction of dimer interaction energies. ${ }^{19}$ The resulting model, SAPTML, was trained to predict the four SAPT0 components for a set of hydrogen-bonded dimers. A consequential design choice of the SAPT-ML model was that the interaction energy could also be partitioned into individual atomic contributions, each of which is a learnable function of an atom's local environment:

$$
\Delta E_{\text {int }}=\sum_{a \in A} \Delta E_{a}+\sum_{b \in B} \Delta E_{b},
$$

where $a$ and $b$ index the atoms of monomers $A$ and $B$ respectively. Eq. 4 is a straightforward intermolecular adaptation of Eq. 3, the standard formulation of ML potentials.

The current work is motivated by the observation that NCIs between molecules are well understood to be approximately a sum of interactions between pairs of atoms. Atomic-pairwise additivity corresponds to a different partition of the interaction energy:

$$
\Delta E_{\text {int }}=\sum_{a \in A} \sum_{b \in B} \Delta E_{a b}
$$

This paradigm is fundamental to NCIs and dates at least as far back as the original Lennard-Jones potential ${ }^{37}$ Pairwise additivity is still the basis of the many popular classical force fields like AMBER, CHARMM, and OPLS 3840 Advanced polarizable force fields such as AMOEBA add small, self-consistent corrections on top of a pairwise additive model ${ }^{41}$ The $\mathrm{D} 3$ dispersion correction of Grimme and coworkers captures the purely quantum mechanical phenomenon of dispersion with yet another pairwise model ${ }^{[42}$ Our group has even developed postprocessing methods for partitioning a calculated SAPT0 interaction energy into both atom and functional group pairs $\underline{43[44}$

In the design of machine learning models, incorporating prior knowledge about the nature of the function to be approximated is a fundamental priority. For example, major advances in the field of computer vision are a result of encoding locality and shift invariance in neural networks (through so-called "convolutional" layers) 4 45/46 Given the overwhelming amount of chemical intuition 
and empirical evidence supporting the pairwise additive nature of NCIs, it seems imperative to incorporate this information into the model. This insight is the motivation behind AP-Net, an atomic pairwise neural network model for interaction energies, developed in this work. Functionally, AP-Net is similar to SAPT-ML in that both models use a geometric description of a molecular dimer to predict an interaction energy. The important difference lies in the atomic-pairwise nature of AP-Net's architecture.

\section{Features}

Regression problems require a careful choice of variables (or features/descriptors) from which a mapping is approximated (or learned) to some desired property (or label). The selection of features can greatly affect both the accuracy of the final model and its ability to generalize well to unseen data. These concerns are particularly relevant for chemical systems, since standard feature engineering techniques don't immediately apply to the unique graph-like structure of molecular geometries. Thus, the representation of chemical data is an essential component of machine learning potentials. Some aspects of useful features can be reasoned about a priori. For one, features should obey the same invariances as the predicted property. For potentials, this means that the features should be invariant to relabeling of identical atoms, molecular translation, and molecular rotation. While many features are used across various atomic potentials, the atomic-pairwise model described in this work necessitates the development of features that can describe a pair of atoms.

To begin with, the atomic pair paradigm has an immediate set of sensible and descriptive features not explicitly available in the original atomic paradigm: $Z_{a}, Z_{b}$, and $r_{a b}$. The existence of simple, qualitatively correct, nonbonded force fields based on these variables suggests that they can be used to account for a large fraction of interaction energies, and we use them as inputs to APNet. However, these three variables alone are not sufficient to describe all of NCIs, as they don't contain information about individual atomic environments or their orientations with respect to the other monomers. Thus, additional descriptors are necessary. For this purpose, the popular atom-centered symmetry function $(\mathrm{ACSF})^{7}$ is reviewed and the new atom-pair symmetry function (APSF) is introduced.

\section{Atom-Centered Symmetry Functions (ACSFs)}

The prediction of pseudo-atomic properties (like charge) and atomic partitions of molecular properties (like energy) are common applications of machine learning to chemistry. As such, much effort has been put into the design of features that encode local atomic environ- ments to use in ML models. In contrast, this work seeks to predict a partitioning of the interaction energy over pairs of atoms, not individual atoms. Nevertheless, a reasonable starting point for a feature to describe a pair of atoms is simply the concatenation of individual atomic features. A well-established descriptor for this purpose is the radial atom centered symmetry function (ACSF) of Behler and Parinello ${ }^{7}$ A radial ACSF of atom $i\left(G_{i}^{r a d}\right)$ describes the atom's local environment in terms of the radial distribution of neighboring nuclei:

$$
G_{i}^{r a d}(\mu, \eta, Z)=\sum_{j \neq i} e^{-\eta\left(r_{i j}-\mu\right)^{2}} f_{c}\left(r_{i j}\right) \delta_{Z Z_{j}}
$$

$$
f_{c}\left(r_{i j}\right)= \begin{cases}\frac{1}{2}\left(\cos \left(\frac{\pi r_{i j}}{r_{c}}\right)+1\right) & r_{i j} \leq r_{c} \\ 0 & r_{i j}>r_{c}\end{cases}
$$

$G_{i}^{r a d}$ encodes the radial density of atom $i$ 's neighbors. The $\delta_{Z Z_{j}}$ term filters only neighboring nuclei with a particular atomic number. The parameters $\mu$ and $\eta$ define the radius and width of a spherical Gaussian shell upon which the neighbor density is projected onto. Lastly, the cutoff function $f_{c}$ ensures locality by removing contributions of neighbors outside of a chosen cutoff radius $r_{c}$. In practice, many radial ACSFs are used to describe the complete radial environment around atom $i$ (i.e. a collection of $G_{i}^{r a d}$ with varying $\mu, \eta$, and $Z$ ). We denote such a collection or vector of ACSFs for a single atom with different $\{(\mu, \eta, Z)\}$ as $\vec{G}_{i}^{r a d}$. Figure 1 depicts individual elements of $\vec{G}_{i}^{r a d}$ for an example system. In the dimer picture, we further indicate that the ACSF vector is formed from only intramonomer neighboring nuclei as $\vec{G}_{a}^{r a d}$ or $\vec{G}_{b}^{r a d}$. It is important to note that, while we chose radial ACSFs to describe the intramolecular environment for their speed and simplicity, any other feature could be substituted or added, including the more expensive angular ACSFs. In particular, the increasingly popular message-passing framework ${ }^{10 / 12[17 / 47}$ could be adapted to the dimer picture and used here.

\section{Atom Pair Symmetry Functions (APSFs)}

The monomer ACSFs $\vec{G}_{a}^{r a d}$ or $\vec{G}_{b}^{r a d}$ as defined above encode an atom's local environment within its respective monomer, containing no information about the identities of, distances from, or orientations to atoms in the other monomer. However, when these monomer ACSFs are combined with $Z_{a}, Z_{b}$, and $r_{a b}$, we can achieve a nearly complete description of a pair of atoms in opposite monomers, lacking only information about intermonomer orientation. A model developed to use only these features would therefore result in an isotropic description of atom-atom interactions. This can be a poor approximation, since the electron density around an atom may be anisotropic (e.g. higher along a bond axis or in the direction of an electron lone pair). 
A

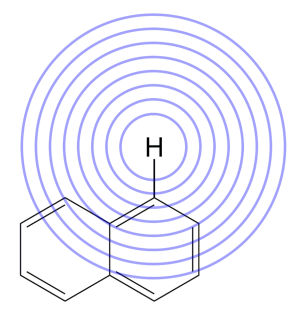

B

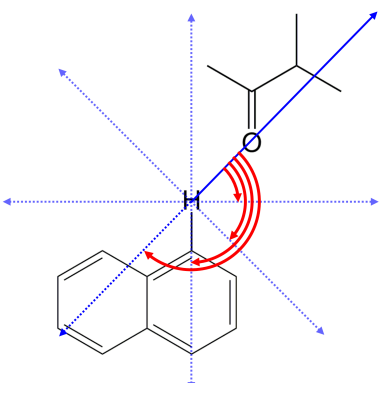

FIG. 1. A 2D illustration of the ACSF and APSF features used in AP-Net. A The local environment of a hydrogen in napthalene $(1-\mathrm{H})$ is described by a set of ACSFs $\left(\vec{G}_{H}^{r a d}\right)$. Each $\mu$ is associated with a distance from the hydrogen, drawn as a blue circle. B The orientation of the same hydrogen's local environment with respect to an oxygen on a different monomer is described by a set of APSFs $\left(\vec{G}_{H(O)}^{\text {ang }}\right)$. Each $\mu$ is associated with a direction from the hydrogen, drawn as a blue ray. The dependence of this direction on the HO intermolecular axis is indicated in red.

Here, we introduce angular atom-pair symmetry functions (APSFs) as a way to account for orientation of atomic environments within the atom-pair paradigm. The angular APSF of an atom $a$ in monomer A with respect to an atom $b$ in monomer $\mathrm{B}$ is defined as:

$$
G_{a(b)}^{a n g}(\mu, \eta, Z)=\sum_{a^{\prime} \in A, a^{\prime} \neq a} e^{-\eta\left(\cos \theta_{a^{\prime} a b}-\mu\right)^{2}} f_{c}\left(r_{a a^{\prime}}\right) \delta_{Z Z_{a^{\prime}}}
$$

where index $a^{\prime}$ runs only over atoms in the same monomer as atom $a$. The angular APSF closely resembles the original radial ACSF, and the two features are compared in Figure 1. In the APSF, atom $a$ is still described by the spatial distribution of its neighboring nuclei within monomer A. The terms $f_{c}\left(r_{a a^{\prime}}\right)$ and $\delta_{Z Z_{a^{\prime}}}$ serve analogous roles as in ACSFs, enforcing spatial locality and filtering out nuclei by atomic number, respectively. The essential difference of this descriptor is the shape of Gaussian that the neighbor density is projected onto, which is now cone-like instead of spherical. The apex of the cone is at nucleus $a$ and the cone is aligned with the $a b$ axis. $\mu$ determines the apex angle of a cone (instead of the radius of a sphere), and $\eta$ is still the Gaussian width. Different values of $\mu$ define cones with different angles. The value $\cos \theta_{a^{\prime} a b}$ is determined by the alignment of the vector $\vec{r}_{a a^{\prime}}$ with the $a b$ axis. The range of reasonable $\mu$ values is the same range as the cosine function, $[-1,1]$. If an ACSF is understood to encode an atom's neighbor density as a function of distance, the APSF can be thought of as encoding an atom's neighbor density as a function of angle or orientation, where an atom in a different monomer is the third point in the angle.

The vector notation used for the ACSFs is also used for APSFs. Note that $\vec{G}_{a(b)}^{a n g}$ is not equal to $\vec{G}_{b(a)}^{a n g}$. The former describes the environment of atom $a$ in monomer $A$ with respect to the orientation of atom $b$ in monomer $B$ while the latter describes the environment of $b$ with re-

spect to the orientation of $a$. The intent behind the APSF descriptor is to explicitly decouple intermonomer orientation from intermonomer distance, which is already captured by using $r_{a b}$ as a feature. Without the APSF, APNet could not be able to account for atomic anisotropy. It would be possible to use additional hyperparameters in the APSF, for example by including an additional spherical Gaussian shell to project $r_{a a^{\prime}}$ onto with its own $\mu$ and $\eta$, much like an ACSF. However, minimizing the number of necessary features is a worthwhile pursuit if APNet is to be used in applications such as high-throughput screening or molecular dynamics simulations.

\section{Network Architecture, Training, \& Implementation}

A common practice in the design of neural network potentials under the atomic partition is to parameterize a separate neural network for each element (atom type):

$$
\left(\vec{G}_{a}\right) \stackrel{Z_{a}}{\mapsto} E_{a} .
$$

This splitting out of networks has been seemingly necessary to achieve good performance, but is undesirable for a number of reasons. For one, it results in unwieldy implementations that scale in size with the number of treated atom types. The use of separate atomic networks is also not very data-efficient, since generalization across atom types by definition cannot be learned by independent networks. Data-efficiency is particularly important when some atom types are much less common in a dataset. In accordance with recent work on shared-weight models, $\underline{48}$ the proposed AP-Net architecture avoids this problem. For each SAPT component energy $\left(E_{c o m p}\right)$, a single neural network is trained to predict atomic pair partitions $\left(E_{a b, c o m p}\right)$ of the component energy:

$$
\left(\vec{Z}_{a}, \vec{Z}_{b}, \vec{G}_{a}^{r a d}, \vec{G}_{b}^{r a d}, \vec{G}_{a(b)}^{a n g}, \vec{G}_{b(a)}^{a n g}, r_{a b}\right) \stackrel{\text { omp }}{\mapsto} E_{a b, c o m p}
$$

where the notation $(\cdot, \cdot)$ represents concatenation. The vector $\vec{Z}_{i}$ is a concatenation of the atom's atomic number and a binary variable (or one-hot) encoding of the atomic number. (For a model that accommodates $N$ atom types, $\vec{Z}_{i}$ has length $N+1$, and the last $N$ elements are all zeros except for a one in the position corresponding to the current atom type.) The network is also symmetrized with respect to $a$ and $b$ by averaging the output over the two possible orders, ensuring that predictions are independent of the order of the monomers. An important final adjustment to the internal neural network architecture of AP-Net is made to encourage correct asymptotic behavior. Rather than using a raw neural network output as a prediction for $E_{a b, c o m p}$, the output of the last network layer is scaled by $r_{a b}^{-1}$ for every component. This normalization guides predictions to have small magnitude at large interatomic distance. Thus, $r_{a b}$ is both an input to the model and an explicit part of the functional form. 
AP-Net was developed with version 2.1.0 of the TensorFlow library ${ }^{49}$ The model was constructed to handle the six atom types present in the datasets described in Section IIE (H, C, N, O, F, and S). For the ACSF feature, $\eta$ is fixed at 100.0 and $\mu$ varies from $0.8 \AA$ to $5.0 \AA$ in increments of $0.1 \AA$. For the APSF feature, $\eta$ is fixed at 25.0 and $\mu$ varies from -1.0 to 1.0 in increments of 0.1 . The cutoff radius, $r_{c}$ is fixed at $8.0 \AA$. Each network consists of three dense layers of 128 nodes and a final output layer of a single node. As discussed previously, the output layer is scaled by $r_{a b}^{-1}$ to form $E_{a b, c o m p}$. Additionally, both ACSF and APSF vectors are preprocessed with separate dense layers of 100 and 50 nodes, respectively. All layers use the rectified linear activation function, except for the output layer which uses a linear activation function. Each network is trained for 200 epochs using the Adam optimizer with a learning rate of $1.0 \times 10^{-4}$ and a batch size of a single molecule. ${ }^{50}$ While we previously used a multi-target loss function that balanced the accuracy of individual component energies and total interaction energies, here we chose to train networks separately, prohibiting explicit error cancellation. ${ }^{19}$ The training procedure minimizes the mean squared error of the predicted component energy. The network weights resulting in the lowest error on a held-out validation subset of the training data over the 200 epochs are used as in the final model. Eight randomly initialized networks are trained per SAPT component, and AP-Net reports the average prediction of the eight networks. The variance of the ensemble predictions can also be used as an uncertainty metric.

\section{E. Training and Testing Datasets}

The hydrogen-bonded dimer dataset previously developed with SAPT-ML is revisited $\frac{19}{19}$ This dataset features interactions between N-methylacetamide (NMA)a popular model system for peptide bonding and universal hydrogen-bond donor and acceptor-paired with other small hydrogen-bond acceptors and donors. The training data was created by selecting a number of these other molecules, placing them in a favorable hydrogenbonding orientation with NMA, and then procedurally varying the monomer separation and orientation. Intramonomer geometry was also sampled with small, random perturbations from equilibrium so that models trained on the dataset are capable of disentangling the effect of intramolecular geometry on the interaction energy. This was done for 92 small molecules expressing 149 chemically distinguishable donor and acceptor sites hydrogen bonded with NMA in 7784 different configurations. The training data was further supplemented with the 2192 neutral dimers in the sidechain-sidechain interaction (SSI) dataset $[1]$ The testing data consists of NMA in complexation with donors and acceptors absent from the training set, and was either taken from crystallographic data,$\sqrt[5253]{53}$ or, in the case of isoquinolone as an acceptor, generated using the same sampling method as described above for the training data. A subset of these configurations is shown in Figure 2. We will refer to these two datasets of dimers as NMA-training and NMA-testing. Throughout this work, a randomly selected subset of 498 dimers from NMA-training is used for validation as described in Section $\llbracket$ II

Experiments are performed to assess AP-Net's ability to describe a large expanse of the intermolecular PES of a single hydrogen-bonded dimer. We examine the $\mathrm{H}_{2} \mathrm{O}$ dimer and the dependence of the interaction energy on intermonomer separation and orientation. We report improvements on the shape, asymptotic behavior, and smoothness of the neural network PES.

We also test the transferability of AP-Net to the diverse S66x 8 benchmark dataset, which contains 66 small dimers each at 8 different geometries along the radial dissociation curve 21 The dimers consist of small, closedshell, neutral molecules and span a wide range of interaction types, from hydrogen-bonding to $\pi-\pi$ stacking. Of the eight configurations, one is the equilibrium geometry, five are slightly dissociated $(\times 1.05, \times 1.10, \times 1.25, \times 1.50$, and $\times 2.00)$, and two are slightly compressed $(\times 0.95$ and $\times 0.9)$.

\section{RESULTS AND DISCUSSION}

\section{A. NMA Dataset}

We compare the accuracy of AP-Net to the reported accuracy of the original SAPT-ML model. Both models are trained on exactly the same NMA-training dataset, down to the random subset of the data used for validation. The performance of the two models on the NMAtesting dataset is shown in Table [. AP-Net exhibits a significant improvement in the prediction of all four SAPT0 components as well as the total SAPT0 interaction energy. For 8 of the 10 dimers, the AP-Net error is lower for all four energy components and the total SAPT0 energy. One exception is NMA/cyclohexanone, for which only five configurations are present in NMA-testing. With such a small sample, this behavior appears to be an instance of SAPT-ML benefiting from some combination of randomness and fortuitous error cancellation. Also, both models predict this interaction with small errors. The other dimer, 3-methylbutan-2-one features improved predictions for each component, but is narrowly worse at total interaction energies.

Although AP-Net's predictions for all four SAPT0 components are an improvement over SAPT-ML, the relative magnitudes of errors between components are largely the same for the two models. Errors in the prediction of $E_{\text {elst }}$ remain the highest, followed by $E_{\text {exch }}, E_{\text {ind }}$, and finally $E_{d i s p}$. The average component error is partially explained by the range of possible energies for each component. Figure 3 shows that for the NMA-testing dataset, $E_{\text {elst }}$ and $E_{\text {exch }}$ take on a wider range of values 

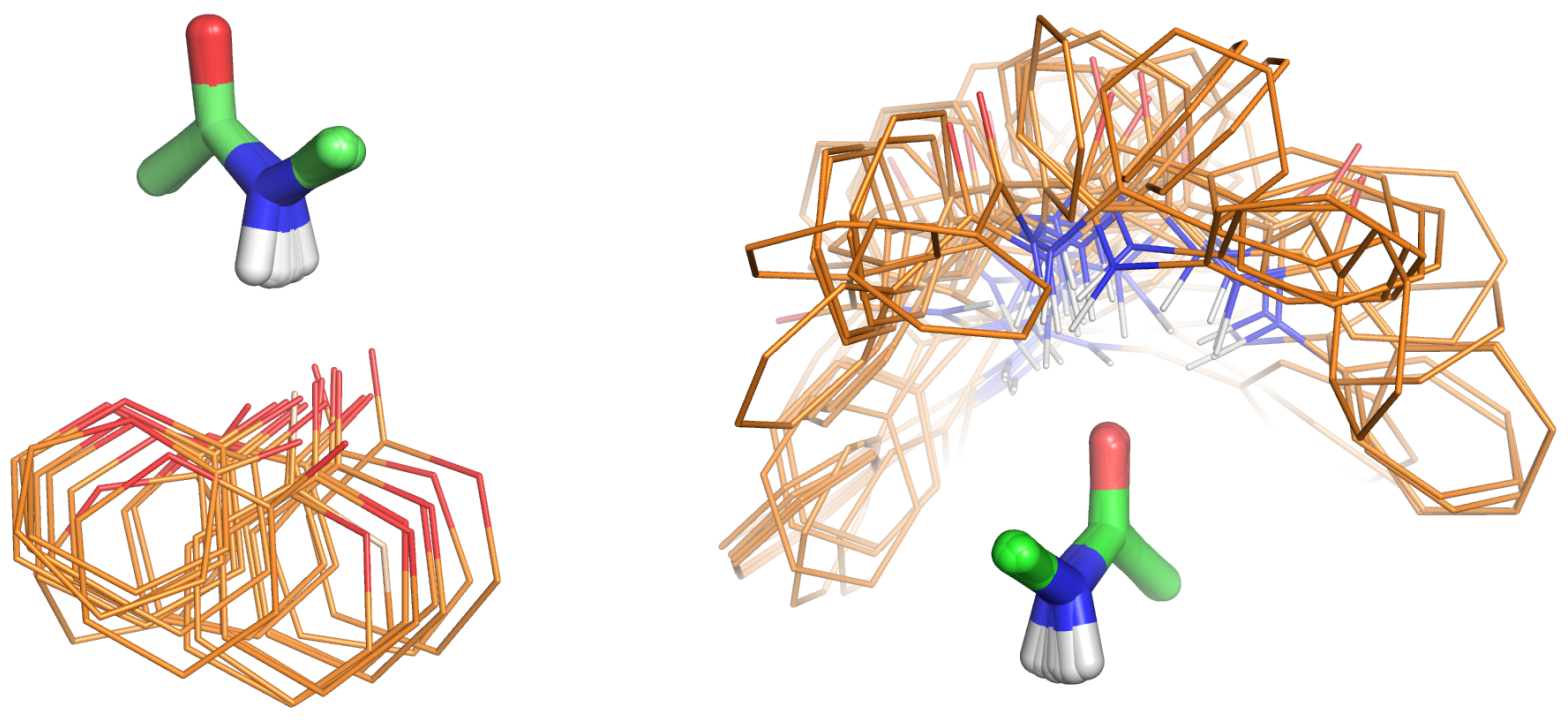

FIG. 2. Overlay of crystallographic hydrogen-bond dimer geometries in the NMA-testing dataset. Left: 19 configurations of tetrahydro-2H-pyran-2-one (acceptor) and NMA (donor). Right: 33 configurations of N-phenylbenzamide (donor) and NMA (acceptor).

TABLE I. Mean absolute error ( $\mathrm{kcal} \mathrm{mol}^{-1}$ ) of SAPT0 interaction energies. Errors are calculated for dimers of NMA paired with various hydrogen-bond acceptors and donors. The accuracy of the current pairwise model (AP-Net) is compared with the model developed in previous work (SAPT-ML). The total error on this test dataset is given both as the average over all configurations (weighted) and the average over the 10 dimer averages (unweighted).

\begin{tabular}{|c|c|c|c|c|c|c|c|}
\hline \multirow[b]{2}{*}{ NMA Pair Monomer } & \multirow[b]{2}{*}{ Role } & \multirow[b]{2}{*}{ Count } & \multicolumn{5}{|c|}{ AP-Net (SAPT-ML) } \\
\hline & & & Electrostatic & Exchange & Induction & Dispersion & Total \\
\hline Isoquinolone $^{a}$ & Acc. & 2052 & $0.15(0.65)$ & $0.08(0.95)$ & $0.03(0.30)$ & $0.02(0.43)$ & $0.15(0.78)$ \\
\hline Tetrahydro- $2 \mathrm{H}$-pyran-2-one & Acc. & 19 & $0.31(1.20)$ & $0.20(0.48)$ & $0.19(0.21)$ & $0.05(0.26)$ & $0.27(1.11)$ \\
\hline N-isopropylacetamide & Don. & 821 & $0.26(0.78)$ & $0.24(0.63)$ & $0.08(0.26)$ & $0.06(0.18)$ & $0.39(0.91)$ \\
\hline Cyclohexanone & Acc. & 5 & $0.56(0.47)$ & $0.32(0.59)$ & $0.14(0.11)$ & $0.05(0.17)$ & $0.43(0.21)$ \\
\hline Aniline & Don. & 188 & $0.51(1.27)$ & $0.19(0.63)$ & $0.06(0.36)$ & $0.02(0.23)$ & $0.59(1.51)$ \\
\hline Naphthalene $(1-\mathrm{H})$ & Don. & 88 & $0.58(0.60)$ & $0.13(0.36)$ & $0.04(0.17)$ & $0.03(0.17)$ & $0.59(0.76)$ \\
\hline 3-methylbutan-2-one & Acc. & 89 & $0.63(1.17)$ & $0.30(1.30)$ & $0.18(0.32)$ & $0.05(0.26)$ & $0.61(0.60)$ \\
\hline N-phenylbenzamide & Don. & 33 & $0.76(0.93)$ & $0.34(1.05)$ & $0.10(0.32)$ & $0.11(0.46)$ & $0.83(1.28)$ \\
\hline Benzimidazole & Don. & 92 & $1.15(1.97)$ & $0.31(1.09)$ & $0.35(0.84)$ & $0.03(0.19)$ & $1.46(1.99)$ \\
\hline Uracil & Don. & 169 & $1.19(2.18)$ & $0.49(0.89)$ & $0.33(0.83)$ & $0.06(0.23)$ & $1.67(2.61)$ \\
\hline Total (weighted) & & 3556 & $0.30(0.84)$ & $0.16(0.85)$ & $0.07(0.33)$ & $0.03(0.33)$ & $0.37(0.97)$ \\
\hline Total (unweighted) & & 3556 & $0.61(1.12)$ & $0.26(0.80)$ & $0.15(0.37)$ & $0.05(0.26)$ & $0.70(1.18)$ \\
\hline
\end{tabular}

${ }^{a}$ All test configurations are extracted from the CSD and the PLDB except for NMA/isoquinolone, for which configurations were procedurally generated as for the training data (see Ref. 19)

(approximately 20 and $30 \mathrm{kcal} \mathrm{mol}^{-1}$ respectively) than $E_{\text {ind }}$ and $E_{\text {disp }}$ (both approximately $10 \mathrm{kcal} \mathrm{mol}^{-1}$ ), a trend that the average errors roughly mirror. However this is not a full explanation, since, for example $E_{\text {elst }}$ errors are actually larger than those of $E_{\text {exch }}$, despite the labels having a smaller range. The existence of relative difficulties in predicting different SAPT components may imply that the physical nature of these interaction types governs how easily they can be modeled. This would explain why AP-Net does a particularly good job at predicting $E_{d i s p}$, with an weighted total MAE of only 0.03 kcal $\mathrm{mol}^{-1}$, an approximately 10-fold improvement over SAPT-ML: dispersion is often well modeled by an atomicpairwise functional form. $\frac{42}{2}$ The use of an individual neu- ral network per component allows the model to separately learn the different physics governing each of them, a unique advantage of training to reproduce SAPT components rather than the total interaction energy. Further tailoring of these individual networks to match known physics could yield additional improvements.

Another similarity between AP-Net and SAPT-ML is the relative difficulty of predicting interactions of different monomers in the NMA-testing dataset. The largest errors are still in the prediction of uracil and benzimidazole, and the smallest errors in the prediction of isoquinolone. We note that the experimentally measured NMA/uracil hydrogen bond lengths are shorter than any of the hydrogen bond lengths in the theoretically gen- 


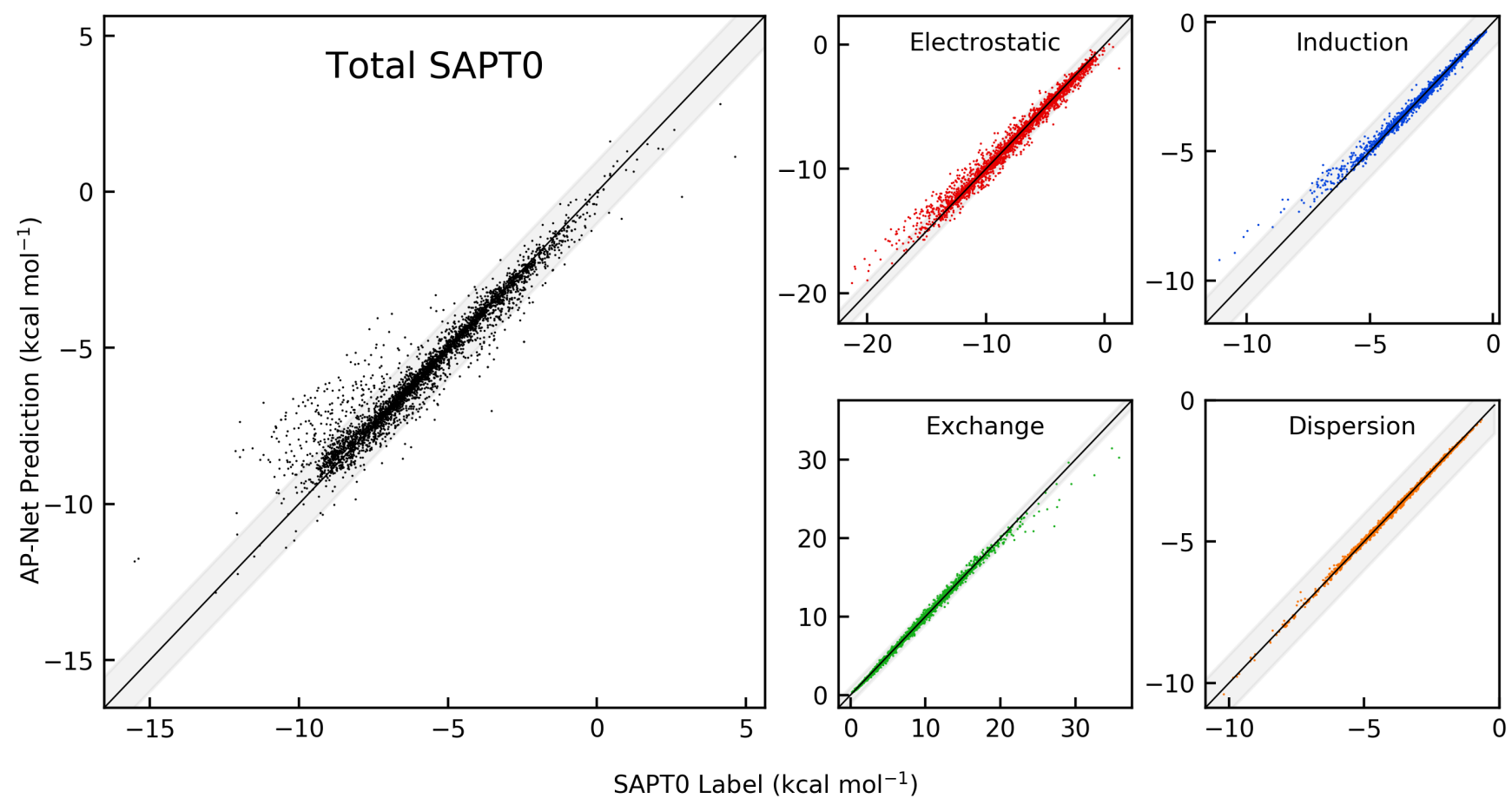

FIG. 3. Correlation between the 3556 true SAPT0 interaction energies and AP-Net predictions for the NMA-testing dataset. The total interaction energy as well as the four components are shown. The shaded region of each plot indicates a $1 \mathrm{kcal}^{\mathrm{mol}}{ }^{-1}$ error margin.

erated NMA-training dataset by up to $0.14 \AA$, so each model is forced to extrapolate its understanding of a hydrogen bond. This is backed up by examining the SAPT0 components for these systems, which are larger in magnitude than any dimer in the training set. The largest exchange component in the experimental test set is $5 \mathrm{kcal} \mathrm{mol}^{-1}$ more repulsive than any exchange component in the training data. AP-Net significantly underestimates $E_{\text {exch }}$ and slightly overestimates $E_{\text {elst }}$ and $E_{\text {ind }}$ for these close contacts, which is evident in Figure 3. These systematic errors are a result of a lack of representative training data, and almost certainly could be eliminated by generating training dimers with shorter hydrogen bonds. The importance of representative data is also underscored by the above average performance on isoquinolone, the only procedurally generated dimer in the NMA-testing dataset.

Figure 4 shows a training saturation curve for AP-Net, illustrating the incremental improvements in accuracy with more training data. The validation error consistently declines for all four SAPT0 components as up to 9000 dimers are used in training. The lack of a plateau is a positive sign, as it suggests that the predictive capability of the relatively simple AP-Net architecture is not yet saturated. Improved performance could be attained simply by adding more training data than is used in this work. The low-data limit is also encouraging. Using only 200 training dimers, AP-Net's predicted total SAPT0 in- teraction energies already reach "chemical accuracy" or $1 \mathrm{kcal} \mathrm{mol}{ }^{-1}$ MAE. The ability to make reasonable predictions with little data illustrates the appropriateness of the atomic-pairwise paradigm.

Another interesting detail in the saturation curve is the existence of a crossover between errors of the electrostatic and exchange components. This can probably be attributed to the variable and long-range nature of electrostatic interactions, which makes prediction slightly more difficult even with many training dimers. The electrostatic energy is the only SAPT component that can be both attractive and repulsive, and its $r^{-1}$ decay is slowest among the four components. This learning curve also further reinforces the observation that the dispersion functional form is well described by a pairwise additive model. The validation MAE for $E_{\text {disp }}$ reaches 0.02 $\mathrm{kcal} \mathrm{mol}^{-1}$ by 5000 training dimers. This error is much smaller than the errors expected in the SAPT0 dispersion energies themselves, as they are computed using only second-order perturbation theory ${ }^{[25}$ Lastly, the parallel behavior of the electrostatic component and total interaction energy errors is particularly striking. Further improvements in the prediction of the SAPT0 interaction energy will necessitate focusing on this component. 


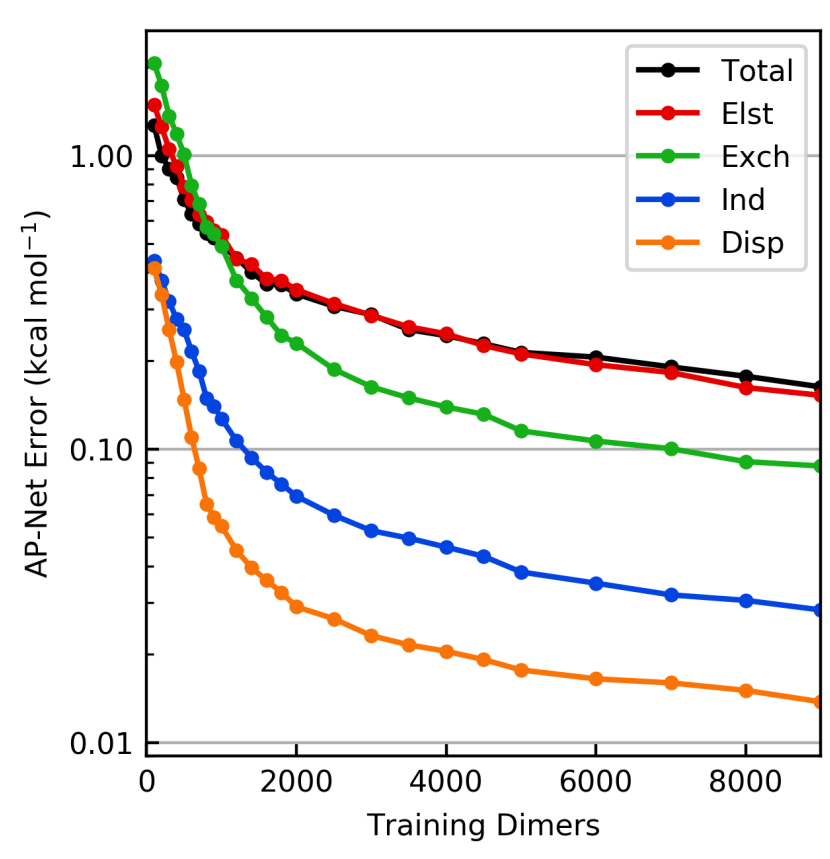

FIG. 4. Saturation curve of AP-Net mean absolute error in the total SAPT0 interaction energy and components. The number of training dimers is varied from 100 to 9000 . The mean absolute error is computed on a randomly selected set of 498 validation dimers from the same distribution as the training data. All models were trained using the same procedure and hyperparameters described in Section IID.
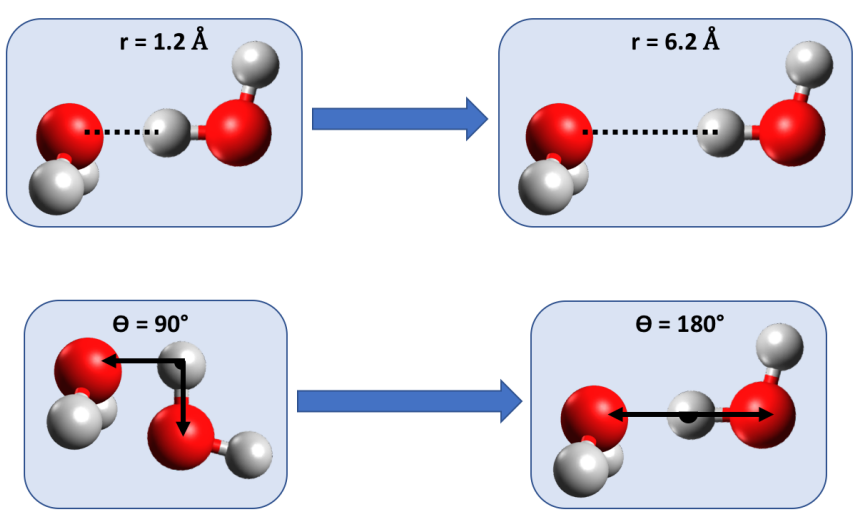

FIG. 5. Depiction of two coordinate scans of the hydrogenbonding $\mathrm{H}_{2} \mathrm{O}$ dimer examined with AP-Net and SAPT-ML. The scans include hydrogen-bond dissociation (top) and rotation (bottom).

\section{B. $\mathrm{H}_{2} \mathrm{O}$ Dimer}

Next, we examine the performance of AP-Net at describing the hydrogen-bonded water dimer. This dimer is absent from the NMA-training dataset, so this experiment is partially a test of AP-Net's ability to generalize to a different intermolecular interaction. More importantly, the $\mathrm{H}_{2} \mathrm{O}$ dimer is of incredible practical relevance and captures the essential, minimal hydrogen bond. We scan the intermolecular dissociation and rotation coordinates of the dimer, illustrated in Figure 5. In generating these coordinates, calculations are performed every $0.01 \AA$ or $0.5^{\circ}$ so that we can assess not only the asymptotic predictions of the models, but also the smoothness of the ML potential. Predicting smooth intermolecular PESs is a necessary requirement for using AP-Net in molecular dynamics or searches for stable intermolecular configurations.

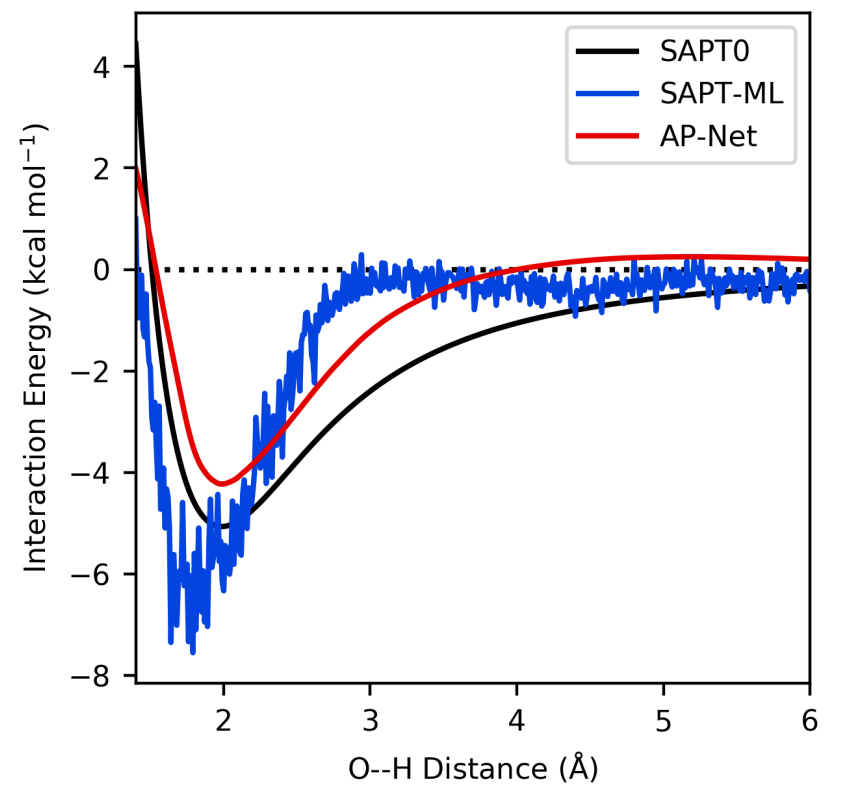

FIG. 6. The total SAPT0 interaction energy of the $\mathrm{H}_{2} \mathrm{O}$ dimer along an intermonomer radial dissociation coordinate is plotted. Predictions of AP-Net and SAPT-ML models are compared to the true SAPT0 values.

The first coordinate probes the dependence of the hydrogen bond on intermonomer separation. Note that the monomer geometries are kept rigid, so any change in APNet's prediction along the scanned coordinate must attributed to changes in the features $r_{a b}, \vec{G}_{a(b)}^{a n g}$, and $\vec{G}_{b(a)}^{a n g}$; the other features $\left(Z_{A}, Z_{B}, \vec{G}_{a}^{r a d}\right.$, and $\left.\vec{G}_{b}^{r a d}\right)$ are constant. The total SAPT0 predictions of AP-Net and SAPT-ML in Figure 6 show that only AP-Net correctly captures the shape of the SAPT0 potential. AP-Net predicts a minimum in the total interaction energy at approximately the correct intermonomer separation, although the strength of the hydrogen bond is underestimated by approximately $1 \mathrm{kcal} \mathrm{mol}^{-1}$. The most striking difference between SAPT-ML and AP-Net, however, is the smoothness of the intermolecular PES. For a given component, neighboring points on the SAPT-ML potential curve fluctuate as much as an entire $\mathrm{kcal} \mathrm{mol}^{-1}$. We believe this noisiness to be a result of the intermolecular descriptors used by SAPT-ML. The availability of actual distances as a feature in AP-Net's atom-pair paradigm 
combined with the carefully designed APSF feature is a significant improvement over the intermolecular ACSFs used in SAPT-ML.

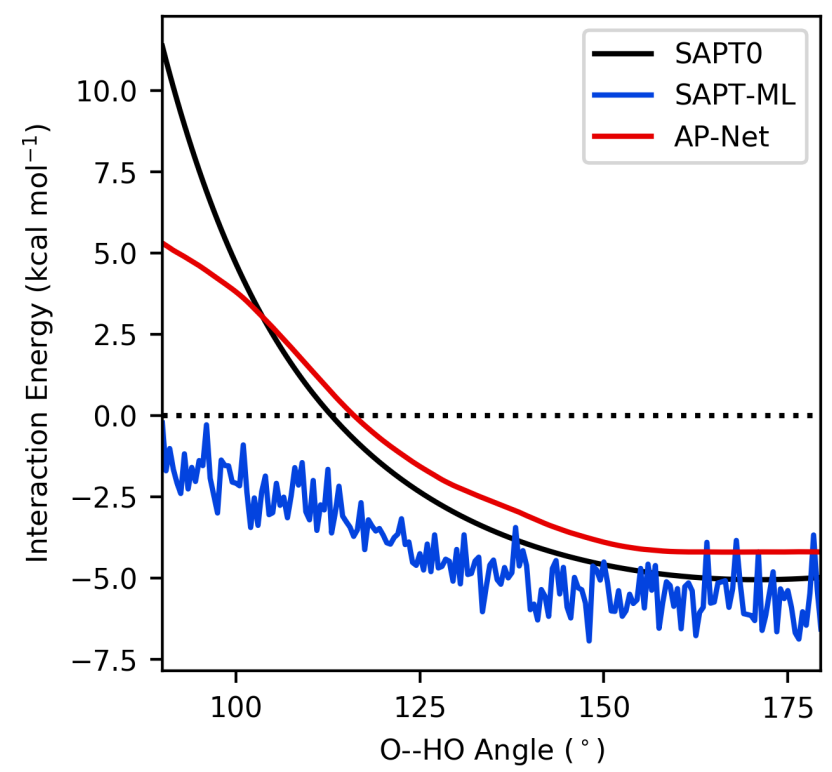

FIG. 7. The total SAPT0 interaction energy of the $\mathrm{H}_{2} \mathrm{O}$ dimer along an intermonomer rotation coordinate is plotted. Predictions of AP-Net and SAPT-ML models are compared to the true SAPT0 values.

The second coordinate, shown in Figure 7 , is a particularly challenging test of an intermolecular potential, as it isolates the angular dependence of the hydrogen bond. Not only are intramonomer geometries rigid, but also $r_{O H}$ is constant value of $1.95 \AA$ for the hydrogenoxygen pair participating in the hydrogen bond interaction. Therefore, most of the change in predicted energy over this coordinate must be accounted for by the new APSFs. AP-Net is still able to correctly predict the trend of the total interaction energy through the rotation. The decline in prediction quality at the smallest angle is a result of a strong repulsive clash between the two oxygen atoms, which become unreasonably close if we follow the curve all of the way to $90^{\circ}$; this repulsive contact is unlike any in the training dimers. The strong curvature of the potential in Figure 6 is an important result, as it shows that AP-Net is able to account for molecular anisotropy, a necessary aspect of accurate NCI potentials. Predictions are still smooth, especially when compared to SAPT-ML.

\section{C. $566 \times 8$}

We have shown AP-Net to be an effective general model for hydrogen-bonded dimers when trained on the NMA-training dataset of similar hydrogen-bonded dimers. However, a useful intermolecular potential must also accurately describe all types of NCIs. This could obviously be accomplished by including additional and diverse training data. Here, we examine transferability from an alternate perspective: How well can the APNet model, a model specialized at hydrogen-bonding, describe other kinds of interactions? This type of analysis is necessary for ML potentials, since we cannot always expect a target system to be well represented in the model's training data. Some combination of interpolation and extrapolation within chemical space will always be necessary.

AP-Net's transferability is assessed by examining performance on the popular S66x8 benchmark dataset for intermolecular interactions. As a comparison, we also test the ANI-1X ML potential on the same benchmark. ANI$1 \mathrm{X}$ is a neural network potential for organic molecules, trained on a dataset of 5.5 million molecular conformations. This dataset includes many noncovalent complexes, making ANI-1X one of the best candidates for a robust intermolecular ML potential. Because AP-Net and ANI-1X are trained to match different levels of theory (SAPT0/jun-cc-pVDZ and $\omega \mathrm{B} 97 \mathrm{X} / 6-31 \mathrm{G}^{*}$ respectively), it would be unfair to compare each model's predictions to a single approximate reference method. Instead, we show predicted interaction energies for each model alongside the reference interaction energy at the same level of theory used to parameterize that model.

Of the 66 different dimers, two pathological but representative cases are shown: the benzene-benzene sandwich dimer in Figure 8 and the cyclopentane-neopentane dimer in Figure 9. Predictions on the remaining 64 dimers can be found in the SI. The intermolecular PESs predicted by AP-Net are at least as accurate and reasonable as ANI-1X's on average. For the benzene dimer, both AP-Net and ANI qualitatively predict the required interaction energy trend-repulsive at close separation and near zero at dissociation, with a slightly attractive minimum somewhere in between. However, ANI-1X predicts a steeper repulsive wall and more separated minimum, potentially as a result of missing the tricky chargepenetration effects known to occur in $\pi-\pi$ interactions. AP-Net closely matches the entire potential, even though this dimer is chemically unlike the NMA hydrogen-bond dimers that make up its training data. AP-Net's behavior is unlikely to be the result of a lucky guess, since the predicted potentials of other $\pi-\pi$ interactions in S66x8 are similarly correct. Presumably, the AP-Net model learned a representation of aromatic carbons via secondary interactions present in hydrogen-bonded dimers such as NMA/benzene.

The cyclopentane-neopentane dimer is an even more extreme comparison, and a good example of the disadvantages of relying solely on error statistics. Although the ANI-1X prediction is fairly accurate in terms of mean absolute error, the shape of the potential is unphysical, containing a spurious minimum and maximum along the coordinate. These artifacts would severely hinder the practical use of this potential. While AP-Net's prediction has only a slightly better MAE than that of ANI-1X, 


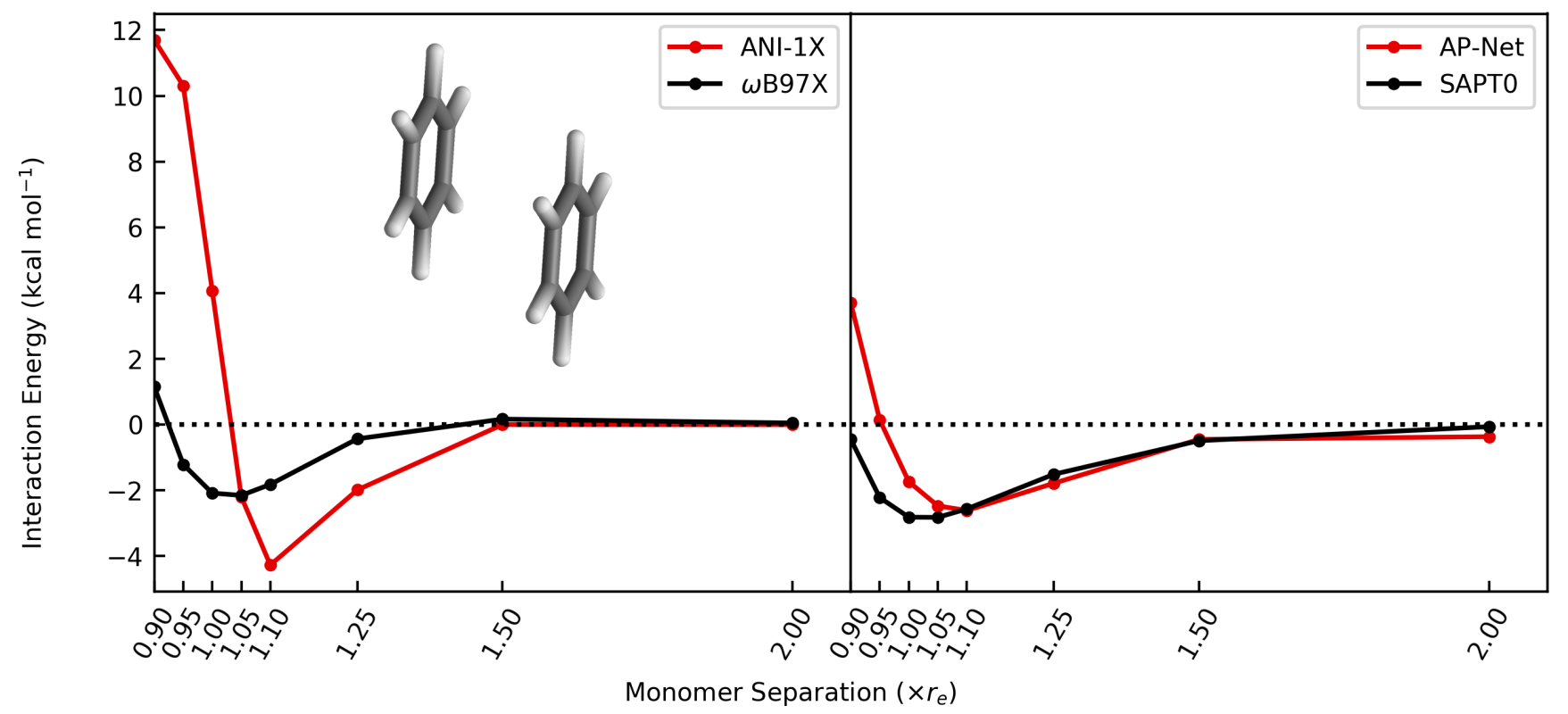

FIG. 8. ANI-1X and AP-Net are evaluated on the 8 geometries of the benzene-benzene sandwich dimer in the S66x8 benchmark. Each ML model is compared to the level of theory used for parameterization: $\omega 97 \mathrm{X} / 6-31 \mathrm{G}^{*}$ for ANI- $1 \mathrm{X}$ and SAPT0/jun-ccpVDZ for AP-Net. The dimer is pictured within the graph.

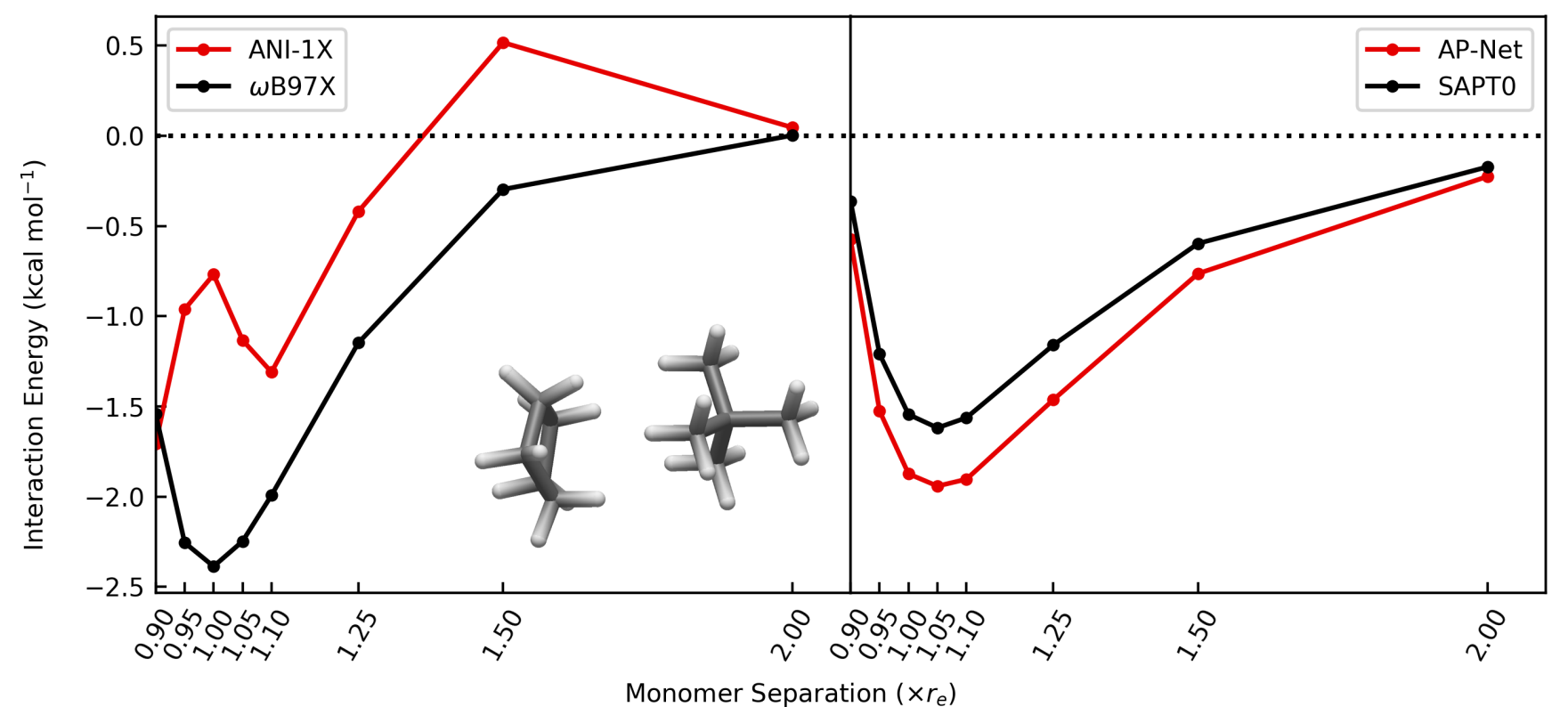

FIG. 9. ANI-1X and AP-Net are evaluated on the 8 geometries of the cyclopentane-neopentane dimer in the S66x 8 benchmark. Each ML model is compared to the level of theory used for parameterization: $\omega$ B97X/6-31G* for ANI-1X and SAPT0/jun-ccpVDZ for AP-Net. The dimer is pictured within the graph.

the predicted potential is parallel to the correct potential, and could be reasonably used for molecular dynamics or a rigid monomer geometry optimization. This occurs despite the fact that the AP-Net model was trained on a much smaller and more chemically homogeneous dataset than ANI-1X. The physical appropriateness of the atomic-pairwise representation is uniquely responsible for the dramatic generalization ability of AP-Net. It should also be emphasized that this type of purely dispersion-bound interaction was not well represented in the NMA-training dataset. 

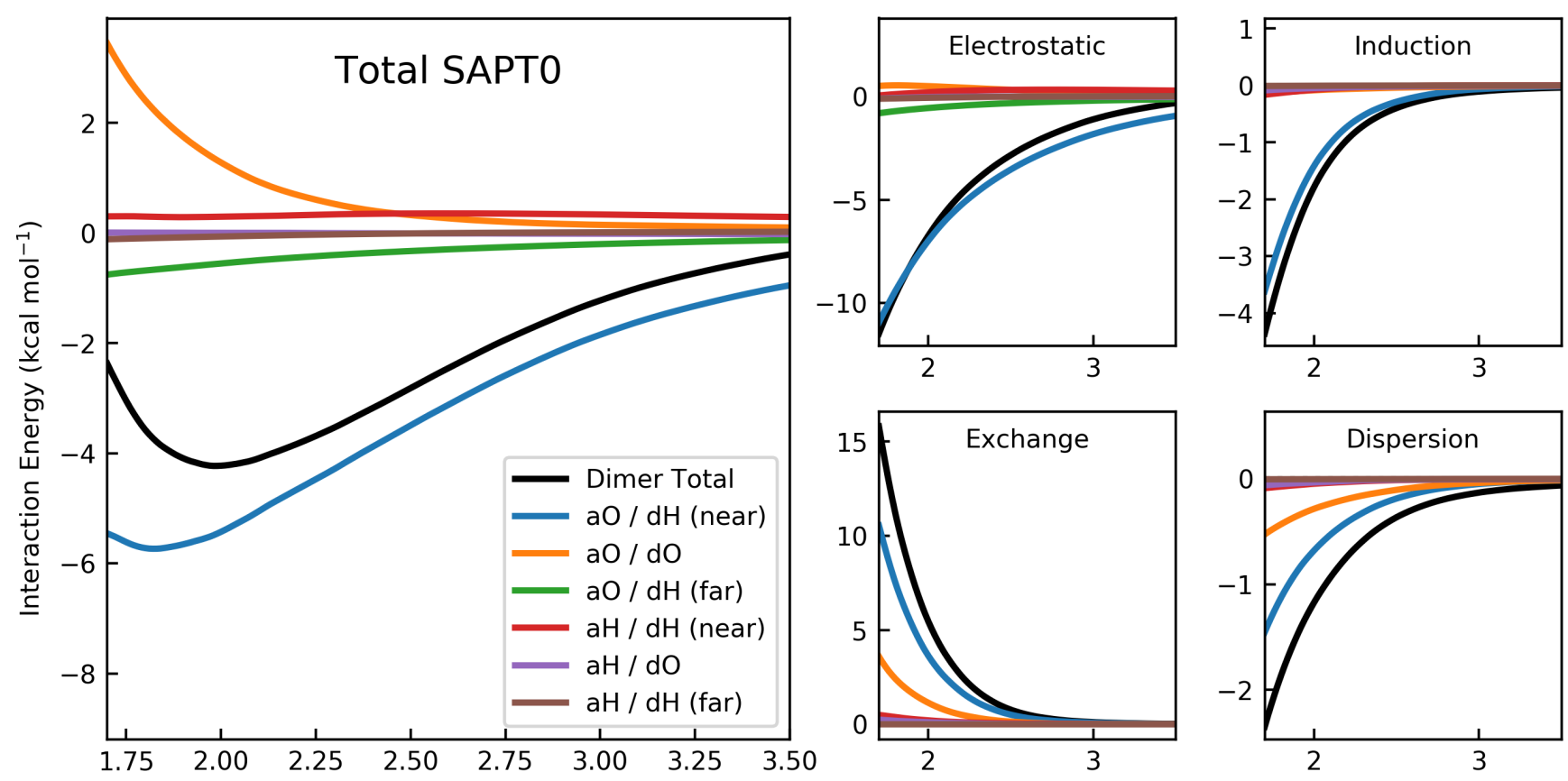

O--H Distance $(\AA)$

FIG. 10. The predicted SAPT0 interaction energy components for the $\mathrm{H}_{2} \mathrm{O}$ dimer along an intermonomer radial dissociation coordinate is shown. In each graph, the total dimer energy predicted by AP-Net is shown in black. The individual atom-pair energy predictions (which sum to the total dimer energy) are also plotted. Every pair includes one atom on the acceptor (a) and donor (d), resulting in six unique atom pairs.

\section{Pairwise Partition Analysis}

So far, the atomic-pairwise energy predictions of APNet are not individually used. They are summed to produce a predicted dimer interaction energy, which is then compared with the $a b$ initio interaction energy. However, the existence of pairwise energy predictions is a unique feature of the atomic-pairwise paradigm, and analyzing these energies can provide insight into the AP-Net model and its representation of chemical systems. For example, AP-Net's pairwise partition of the interaction energy could be compared with empirical force-field models. Pairwise energy predictions of the $\mathrm{H}_{2} \mathrm{O}$ dimer along the radial dissociation coordinate from Section IIIB are shown in Figure 10

As discussed earlier, the predicted SAPT0 interaction energy of this dimer as well as the four SAPT0 components correctly decrease in magnitude along the coordinate. Here, we see that the individual atompair predictions are similarly distance-dependent. This distance-dependence is a good validation of the APNet model, since it matches our intuitive understanding of interactions - any atom-pair interaction should grow weaker as the atoms become farther apart. AP-Net's predictions also qualitatively match the known physics of intermolecular interactions. Electrostatics, the longestrange SAPT component, is correctly predicted to decay slowest of the four components. The pairwise exchange, induction, and dispersion energies have the correct sign. These components are by definition only repulsive or attractive, and we would expect the pairwise energies to match this. It is also interesting to note that the signs of individual electrostatic energy predictions. Interactions between hydrogen and oxygen are attractive, while interactions between two atoms of the same type are repulsive. These predictions reflect the partial-charge picture used in virtually every force-field, even though AP-Net is not trained to predict anything related to electron density. This phenomenon is a natural consequence of the pairwise framework and is an exciting result, since it suggests that AP-Net contains some fundamental representation of the actual physics occurring in intermolecular interactions. This analysis of pairwise energies shows that APNet's predictions are uniquely physically interpretable, and it works towards countering the longstanding "blackbox" criticism of ML models.

In Figure ??, the interpretation of pairwise energies is taken a step further by comparing AP-Net's predictions to those of an $a b$ initio force field developed by Van Vleet et al. .32 . The force field uses atomic dispersion coefficients $\left(C_{6}, C_{8}, C_{10}, C_{12}\right)$ computed from $\mathrm{H}_{2} \mathrm{O}$ monomer frequency-dependent polarizability tensors. Because this force field is fit to DFT-SAPT while AP-Net is fit to SAPT0, complete agreement between the two predictions 




FIG. 11. The predicted SAPT0 dispersion energy components for the $\mathrm{H}_{2} \mathrm{O}$ dimer along an intermonomer radial dissociation coordinate is shown. Predictions of AP-Net (solid line) are contrasted with those of the ab initio force field developed by Van Vleet et al. ${ }^{32}$ (dotted line). In each graph, the total dimer energy predicted by AP-Net is shown in black. The individual atom-pair energy predictions (which sum to the total dimer energy) are also plotted. Every pair includes one atom on the acceptor (a) and donor (d), resulting in six unique atom pairs.

is not expected. Still, the total dimer dispersion energy predicted by AP-Net and the force field are exceedingly close across the entire dissociation coordinate. Although the two predictions agree on the value of $E_{\text {disp }}$, they differ significantly in the atomic-pairwise partition of $E_{\text {disp }}$. AP-Net attributes most of the interaction energy to the close oxygen-hydrogen pair, while the force field assigns an approximately equal split between the close oxygenhydrogen pair and the oxygen-oxygen pair. The two partitionings are not completely irreconcilable, as they both yield the same ordering of pairwise interactions by magnitude. The qualitative agreement between AP-Net and the force field further validates the physical grounding of the AP-Net model. The close correspondence of AP-Net to an $a b$ initio force field is something very few (if any) ML models can claim. AP-Net's predictions could be used to extract a different set of dispersion coefficients. This comparison also illustrates an important lesson in using any sort of energy partition, which is that there is not a singular 'correct' partition.

\section{CONCLUSIONS}

The development of ML potentials is a rapidly evolving endeavor, as evidenced by increasingly technical model architectures, more exhaustively constructed datasets, and lower reported errors on common benchmark tasks. As the field progresses, assessing and developing ML potentials for practical use will become even more important. One such practical application is the quantitative description of of NCIs, where estimated PESs must be smooth and physically reasonable. Although our previously developed SAPT-ML intermolecular potential obtained average errors near chemical accuracy, an investigation of the model's predictions revealed serious shortcomings in the estimated PESs.

In order to address these concerns, here we propose a different formulation of ML potentials specific to the intermolecular case. Instead of the usual atomic partition of energy central to nearly all ML models, the new formulation substitutes an atomic-pairwise partition of interaction energies. Although the energy partition used in any ML potential is arbitrary, an atomic-pairwise partition is decidedly more physically motivated, and therefore it should improve the accuracy and generalizability of any ML potential that make use of it. To test this claim we introduce AP-Net, an atomic-pairwise neural network model for the prediction of interaction energies. New ML descriptors that efficiently represent of a pair of atoms are also developed for AP-Net, including an APSF feature that captures the orientation dependence of monomers, a key aspect of NCIs.

AP-Net is applied to the SAPT0 hydrogen-bonding task developed with the atomic SAPT-ML model, and we find that the new atomic-pairwise model yields dramatic improvements in both the accuracy of single-point energies and the smoothness of predicted potentials. On an experimental NMA hydrogen-bond test dataset, AP-Net predicts a smooth and relatively accurate intermolecular PES for the $\mathrm{H}_{2} \mathrm{O}$ dimer, correctly describing both the radial and angular dependence of a hydrogen-bond, after being trained on a dataset entirely absent of this dimer.

This hydrogen-bond specialized AP-Net model also shows a surprising ability to generalize across chemicalspace, achieving a mean absolute error of $1.1 \mathrm{kcal} \mathrm{mol}^{-1}$ on the entire S66x8 noncovalent interaction benchmark. This level of accuracy surpasses that of the general universal neural network potential ANI-1X. AP-Net predicts more physically reasonable potentials than the aforementioned ML potential on this benchmark, free of spurious optima, while using fewer and less diverse training data.

The ability of AP-Net to make reasonable predictions for disparate interaction types, i.e. extrapolation across chemical space, is an incredibly important characteristic of an ML potential. This behavior is a direct consequence of the atomic-pairwise framework, which provides the model with a physically motivated inductive bias. Put another way, AP-Net regresses over many atomatom interactions, while an atomic model like SAPT-ML 
regresses over fewer atom-monomer interactions. The atom-atom 'chemical-space' is much smaller than the atom-monomer 'chemical-space', making the regression problem simpler and the predictions more accurate under an atomic-pairwise energy partition.

Future work related to AP-Net will focus on using a larger, more chemically diverse dataset that samples a greater expanse of the intermolecular PES. Curating more accurate interaction energies than the SAPT0 labels used here is also pertinent, given that AP-Net's errors with respect to SAPT0 are on average smaller than the errors in the ab initio SAPT0 calculation. Lastly, we note that the general atomic-pairwise framework advocated for in this work could be easily adapted to work with other features, model architectures, and learning tasks.

\section{SUPPLEMENTARY MATERIAL}

The supplementary material contains additional analysis of AP-Net's predictions on the NMA-testing dataset. Predictions on all 66 radial coordinates in the $\mathrm{S} 66 \mathrm{x} 8$ benchmark are also included.

\section{DATA AVAILABILITY}

Code to create an AP-Net model, all datasets used in this work, and the trained AP-Net model used in this work will be included with the final manuscript.

\section{ACKNOWLEDGMENTS}

The authors gratefully acknowledge financial support from Bristol Myers Squibb, the U.S. National Science Foundation (Grant No. CHE-1566192), and the US Department of Energy (DOE) Office of Science, Office of Basic Energy Sciences, Computational Chemical Sciences (CCS) Research Program under work proposal number AL-18-380-057

The following article has been submitted to The Journal of Chemical Physics. After it is published, it will be found at https://aip.scitation.org/journal/jcp. 
${ }^{1}$ M. Rupp, O. A. von Lilienfeld, and K. Burke, J. Chem. Phys. 148, 241401 (2018)

${ }^{2}$ A. C. Mater and M. L. Coote, J. Chem. Inf. Model. 59, 2545 (2019)

${ }^{3}$ T. Bereau, D. Andrienko, and O. A. von Lilienfeld, J. Chem. Theory Comput. 11, 3225 (2015).

${ }^{4}$ P. L. Houston, A. Nandi, and J. M. Bowman, J. Phys. Chem. Lett. 10, 5250 (2019)

${ }^{5}$ F. M. Paruzzo, A. Hofstetter, F. Musil, S. De, M. Ceriotti, and L. Emsley, Nat. Commun. 9, 4501 (2018).

${ }^{6}$ J. Behler and M. Parrinello, Phys. Rev. Lett. 98, 146401 (2007)

${ }^{7}$ J. Behler, J. Chem. Phys. 134, 074106 (2011)

${ }^{8}$ K. T. Schütt, F. Arbabzadah, S. Chmiela, K. R. Müller, and

A. Tkatchenko, Nat. Commun. 8, 13890 (2017)

${ }^{9}$ J. S. Smith, O. Isayev, and A. E. Roitberg, Chem. Sci. 8, 3192 (2017)

${ }^{10}$ N. Lubbers, J. S. Smith, and K. Barros, J. Chem. Phys. 148, 241715 (2018).

${ }^{11}$ R. T. McGibbon, A. G. Taube, A. G. Donchev, K. Siva, F. Hernández, C. Hargus, K.-H. Law, J. L. Klepeis, and D. E. Shaw, J. Chem. Phys. 147, 161725 (2017)

${ }^{12}$ K. T. Schütt, H. E. Sauceda, P.-J. Kindermans, A. Tkatchenko, and K.-R. Müller, J. Chem. Phys. 148, 241722 (2018)

${ }^{13}$ K. Yao, J. E. Herr, D. Toth, R. Mckintyre, and J. Parkhill, Chem. Sci. 9, 2261 (2018)

${ }^{14}$ J. S. Smith, B. Nebgen, N. Lubbers, O. Isayev, and A. E. Roitberg, J. Chem. Phys. 148, 241733 (2018).

${ }^{15}$ T. Bereau, R. A. DiStasio, A. Tkatchenko, and O. A. von Lilienfeld, J. Chem. Phys. 148, 241706 (2018)

${ }^{16}$ J. S. Smith, B. T. Nebgen, R. Zubatyuk, N. Lubbers, C. Devereux, K. Barros, S. Tretiak, O. Isayev, and A. E. Roitberg, Nat. Commun. 10, 2903 (2019)

${ }^{17}$ O. T. Unke and M. Meuwly, J. Chem. Theory Comput. 15, 3678 (2019)

${ }^{18}$ R. Zubatyuk, J. S. Smith, J. Leszczynski, and O. Isayev, Sci. Adv. 5, eaav6490 (2019)

${ }^{19}$ D. P. Metcalf, A. Koutsoukas, S. A. Spronk, B. L. Claus, D. A. Loughney, S. R. Johnson, D. L. Cheney, and C. D. Sherrill, J. Chem. Phys. 152, 074103 (2020)

${ }^{20}$ S. Chmiela, A. Tkatchenko, H. E. Sauceda, I. Poltavsky, K. T. Schütt, and K.-R. Müller, Sci. Adv. 3, e1603015 (2017)

${ }^{21}$ J. Řezáč, K. E. Riley, and P. Hobza, J. Chem. Theory Comput. 7, $2427(2011)$

${ }^{2 z}$ B. Jeziorski, R. Moszynski, and K. Szalewicz, Chem. Rev. 94, 1887 (1994)

${ }^{23}$ K. Szalewicz, Wiley Interdiscip. Rev. Comput. Mol. Sci. 2, 254 (2011)

${ }^{24}$ K. Patkowski, Wiley Interdiscip. Rev. Comput. Mol. Sci. (2019), 10.1002/wcms.1452

${ }^{20}$ T. M. Parker, L. A. Burns, R. M. Parrish, A. G. Ryno, and C. D. Sherrill, J. Chem. Phys. 140, 094106 (2014)

${ }^{26}$ R. M. Parrish, D. F. Sitkoff, D. L. Cheney, and C. D. Sherrill, Chem.: Eur. J. 23, 7887 (2017)

${ }^{27}$ J. G. McDaniel and J. R. Schmidt, J. Phys. Chem. A 117, 2053 (2013)

${ }^{28}$ J. G. McDaniel and J. R. Schmidt, Annu. Rev. Phys. Chem. 67, 467 (2016)

${ }^{29}$ J. A. Rackers, Q. Wang, C. Liu, J.-P. Piquemal, P. Ren, and J. W. Ponder, Phys. Chem. Chem. Phys. 19, 276 (2017)

${ }^{30}$ J. A. Rackers, C. Liu, P. Ren, and J. W. Ponder, J. Chem. Phys. 149, 084115 (2018)

${ }^{31}$ J. A. Rackers and J. W. Ponder, J. Chem. Phys. 150, 084104 (2019)

${ }^{32}$ M. J. Van Vleet, A. J. Misquitta, A. J. Stone, and J. R. Schmidt, J. Chem. Theory Comput. 12, 3851 (2016)

${ }^{33}$ M. J. Van Vleet, A. J. Misquitta, and J. R. Schmidt, J. Chem. Theory Comput. 14, 739 (2018)

${ }^{34}$ M. Tafipolsky and K. Ansorg, J. Chem. Theory Comput. 12, $1267(2016)$
${ }^{35}$ E. G. Hohenstein and C. D. Sherrill, J. Chem. Phys. 132, 184111 (2010)

${ }^{36}$ E. G. Hohenstein, R. M. Parrish, C. D. Sherrill, J. M. Turney, and H. F. Schaefer, J. Chem. Phys. 135, 174107 (2011)

37 J. E. Jones, Proc. R. Soc. Lond. A 106, 463 (1924)

${ }^{38}$ D.A. Case, I.Y. Ben-Shalom, S.R. Brozell, D.S. Cerutti, T.E. Cheatham, III, V.W.D. Cruzeiro, T.A. Darden, R.E. Duke, D. Ghoreishi, M.K. Gilson, H. Gohlke, A.W. Goetz, D. Greene, R Harris, N. Homeyer, S. Izadi, A. Kovalenko, T. Kurtzman, T.S. Lee, S. LeGrand, P. Li, C. Lin, J. Liu, T. Luchko, R. Luo, D.J. Mermelstein, K.M. Merz, Y. Miao, G. Monard, C. Nguyen, H. Nguyen, I. Omelyan, A. Onufriev, F. Pan, R. Qi, D.R. Roe, A. Roitberg, C. Sagui, S. Schott-Verdugo, J. Shen, C.L. Simmerling, J. Smith, R. Salomon-Ferrer, J. Swails, R.C. Walker, J. Wang, H. Wei, R.M. Wolf, X. Wu, L. Xiao, D.M. York and P.A. Kollman (2018), AMBER 2018, University of California, San Francisco.

${ }^{39}$ B. R. Brooks, C. L. Brooks, A. D. Mackerell, L. Nilsson, R. J. Petrella, B. Roux, Y. Won, G. Archontis, C. Bartels, S. Boresch, A. Caflisch, L. Caves, Q. Cui, A. R. Dinner, M. Feig, S. Fischer, J. Gao, M. Hodoscek, W. Im, K. Kuczera, T. Lazaridis, J. Ma, V. Ovchinnikov, E. Paci, R. W. Pastor, C. B. Post, J. Z. Pu, M. Schaefer, B. Tidor, R. M. Venable, H. L. Woodcock, X. Wu, W. Yang, D. M. York, and M. Karplus, J. Comput. Chem. 30, 1545 (2009)

${ }^{40}$ E. Harder, W. Damm, J. Maple, C. Wu, M. Reboul, J. Y. Xiang, L. Wang, D. Lupyan, M. K. Dahlgren, J. L. Knight, J. W. Kaus, D. S. Cerutti, G. Krilov, W. L. Jorgensen, R. Abel, and R. A. Friesner, J. Chem. Theory Comput. 12, 281 (2015)

${ }^{41}$ C. Zhang, C. Lu, Z. Jing, C. Wu, J.-P. Piquemal, J. W. Ponder, and P. Ren, J. Chem. Theory Comput. 14, 2084 (2018)

${ }^{42}$ S. Grimme, J. Antony, S. Ehrlich, and H. Krieg, J. Chem. Phys. 132, $154104(2010)$

${ }^{43}$ R. M. Parrish and C. D. Sherrill, J. Chem. Phys. 141, 044115 (2014)

${ }^{44}$ R. M. Parrish, T. M. Parker, and C. D. Sherrill, J. Chem. Theory Comput. 10, 4417 (2014)

${ }^{45}$ K. Fukushima, Biol. Cybern. 36, 193 (1980)

${ }^{46}$ Y. LeCun, B. Boser, J. S. Denker, D. Henderson, R. E. Howard, W. Hubbard, and L. D. Jackel, Neural Comput. 1, 541 (1989)

${ }^{47}$ J. Gilmer, S. S. Schoenholz, P. F. Riley, O. Vinyals, and G. E. Dahl, in Proceedings of the 34th International Conference on Machine Learning Proceedings of Machine Learning Research, Vol. 70, edited by D. Precup and Y. W. Teh (PMLR, International Convention Centre, Sydney, Australia, 2017) pp. 12631272.

${ }^{48}$ T. A. Profitt and J. K. Pearson, Phys. Chem. Chem. Phys. 21, 26175 (2019)

${ }^{49}$ M. Abadi, A. Agarwal, P. Barham, E. Brevdo, Z. Chen, C. Citro, G. S. Corrado, A. Davis, J. Dean, M. Devin, S. Ghemawat, I. Goodfellow, A. Harp, G. Irving, M. Isard, Y. Jia, R. Jozefowicz, L. Kaiser, M. Kudlur, J. Levenberg, D. Mane, R. Monga, S. Moore, D. Murray, C. Olah, M. Schuster, J. Shlens, B. Steiner, I. Sutskever, K. Talwar, P. Tucker, V. Vanhoucke, V. Vasudevan, F. Viegas, O. Vinyals, P. Warden, M. Wattenberg, M. Wicke, Y. Yu, and X. Zheng, "TensorFlow: Large-Scale Machine Learning on Heterogeneous Distributed Systems," (2016).

${ }^{50} \mathrm{D}$. P. Kingma and J. Ba, in 3rd International Conference on Learning Representations, ICLR 2015, San Diego, CA, USA, May 7-9, 2015, Conference Track Proceedings (2015) p. 13.

${ }^{51}$ L. A. Burns, J. C. Faver, Z. Zheng, M. S. Marshall, D. G. A. Smith, K. Vanommeslaeghe, A. D. MacKerell, K. M. Merz, and C. D. Sherrill, J. Chem. Phys. 147, 161727 (2017)

${ }^{52}$ C. R. Groom, I. J. Bruno, M. P. Lightfoot, and S. C. Ward, Acta Crystallogr. B 72, 171 (2016)

${ }^{5}$ Schrödinger Release 2018-4, Protein-Ligand Database (PLDB), Schrödinger, LLC, New York, NY, 2019. 


\section{Supplementary Material to}

AP-Net: An atomic-pairwise neural network for smooth and transferable interaction potentials

Zachary L. Glick, ${ }^{1}$ Derek P. Metcalf, ${ }^{1}$ Alexios Koutsoukas, ${ }^{2}$ Steven A. Spronk, ${ }^{2}$ Daniel

L. Cheney, ${ }^{2}$ and C. David Sherrill ${ }^{1}$

${ }^{1)}$ Center for Computational Molecular Science and Technology,

School of Chemistry and Biochemistry, and School of Computational Science

and Engineering, Georgia Institute of Technology, Atlanta, Georgia 30332-0400,

USA

${ }^{2)}$ Molecular Structure and Design, Bristol Myers Squibb Company, P. O. Box 5400,

Princeton, NJ 08543 


\section{S1: Additional Error Statistics on NMA-Testing}
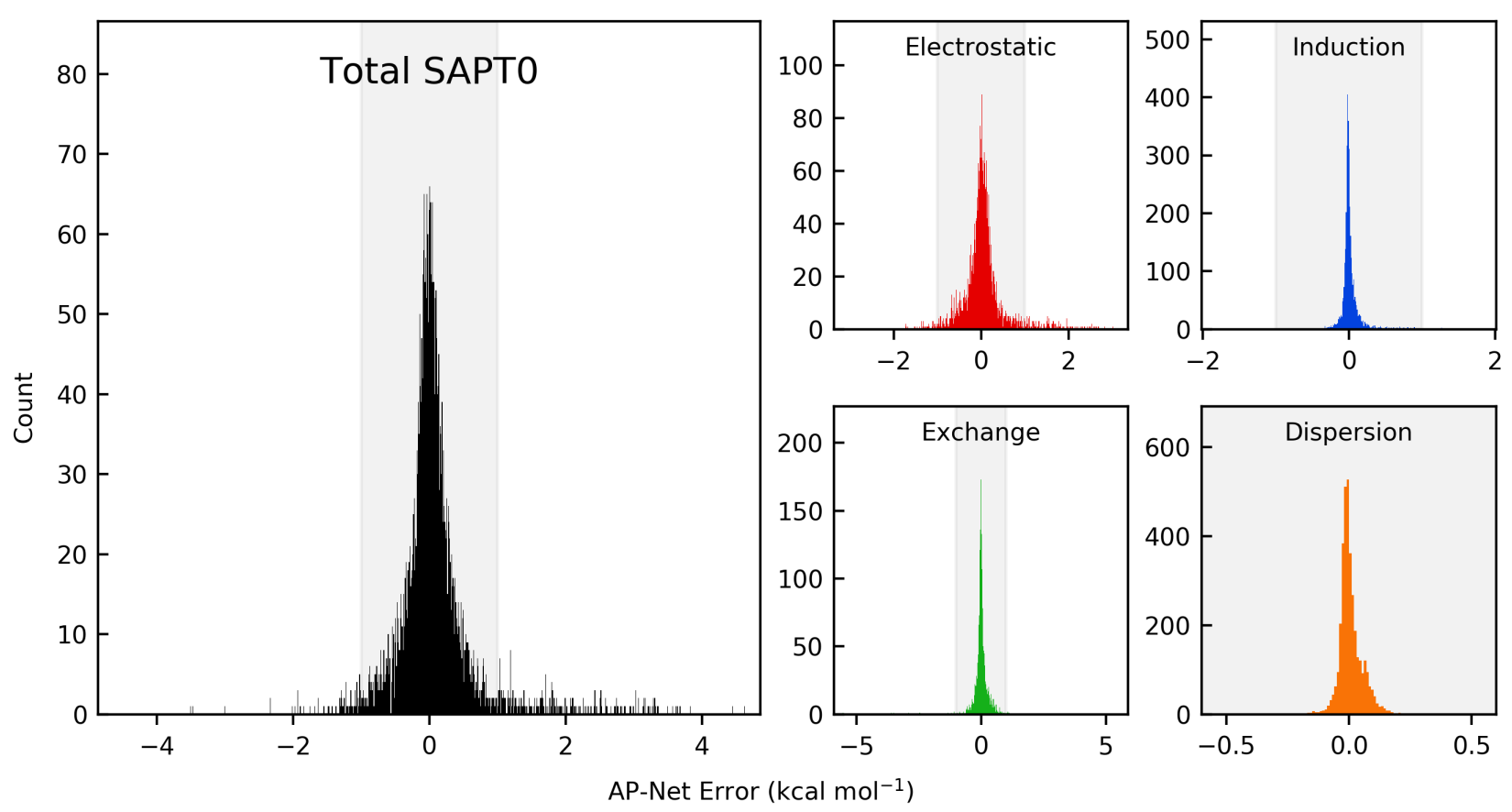

FIG. S1. Error distribution of AP-Net predictions for the NMA-testing dataset. Errors in the total interaction energy as well as the four components are shown. The shaded region of each plot indicates a $1 \mathrm{kcal} \mathrm{mol}^{-1}$ error margin. The entire dispersion error distribution falls within this margin. 


\section{S2: Full S66x8 Results}

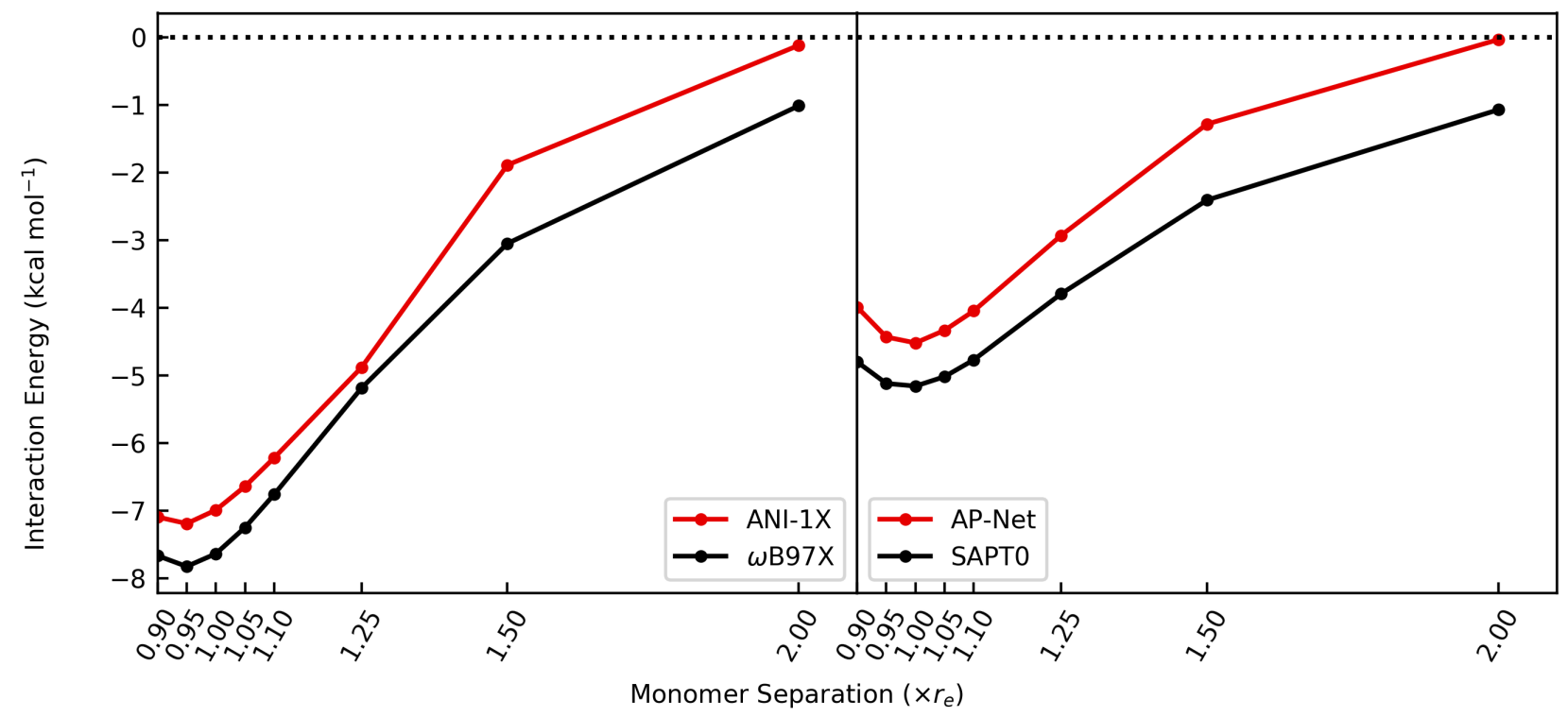

FIG. S2. ANI-1X and AP-Net are evaluated on the 8 geometries of the WaterWater dimer in the S66x8 benchmark. Each ML model is compared to the level of theory used for parameterization: $\omega \mathrm{B} 97 \mathrm{X} / 6-31 \mathrm{G}^{*}$ for ANI-1X and SAPT0/jun-cc-pVDZ for AP-Net. 


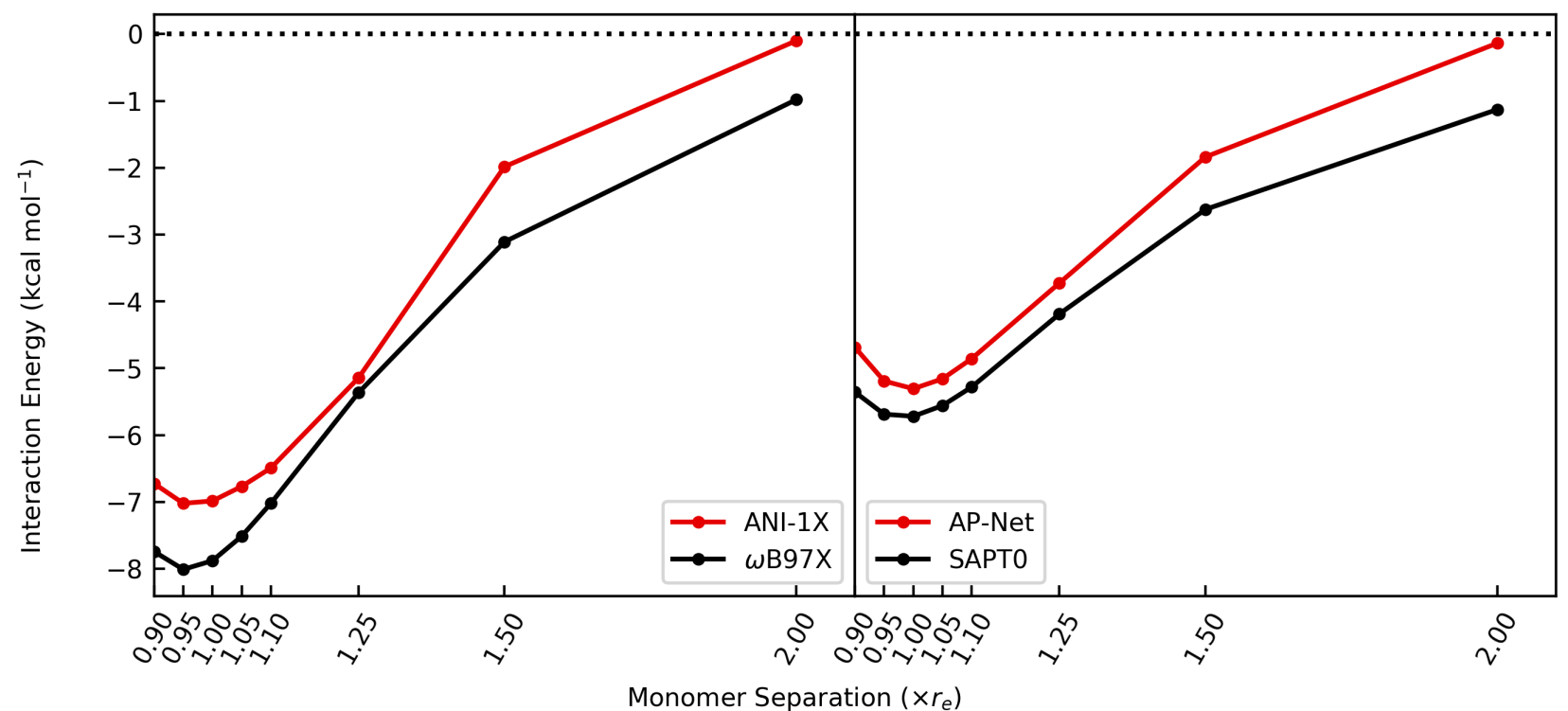

FIG. S3. ANI-1X and AP-Net are evaluated on the 8 geometries of the WaterMeOH dimer in the S66x 8 benchmark. Each ML model is compared to the level of theory used for parameterization: $\omega \mathrm{B} 97 \mathrm{X} / 6-31 \mathrm{G}^{*}$ for ANI-1X and SAPT0/jun-cc-pVDZ for AP-Net.

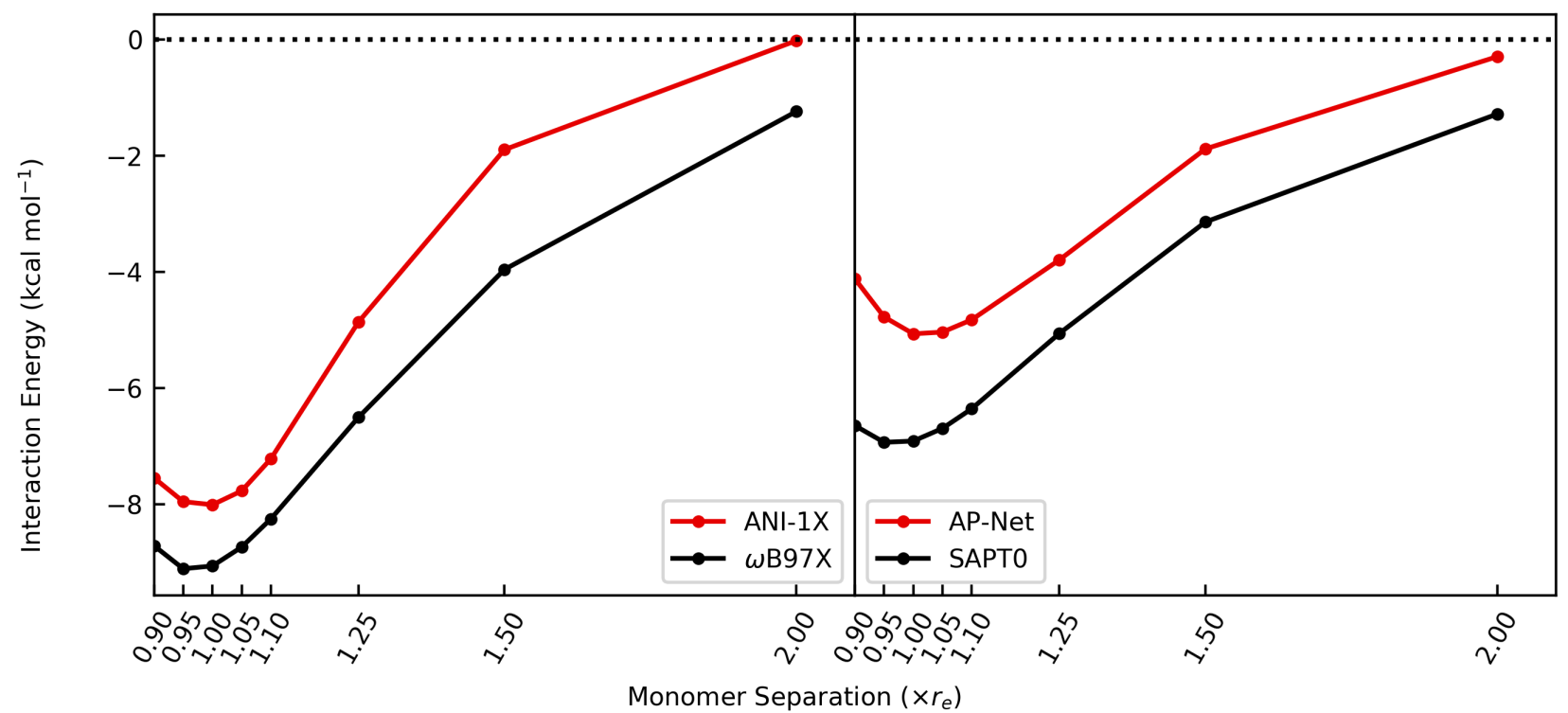

FIG. S4. ANI-1X and AP-Net are evaluated on the 8 geometries of the WaterMeNH2 dimer in the S66x 8 benchmark. Each ML model is compared to the level of theory used for parameterization: $\omega \mathrm{B} 97 \mathrm{X} / 6-31 \mathrm{G}^{*}$ for ANI-1X and SAPT0/jun-cc-pVDZ for AP-Net. 


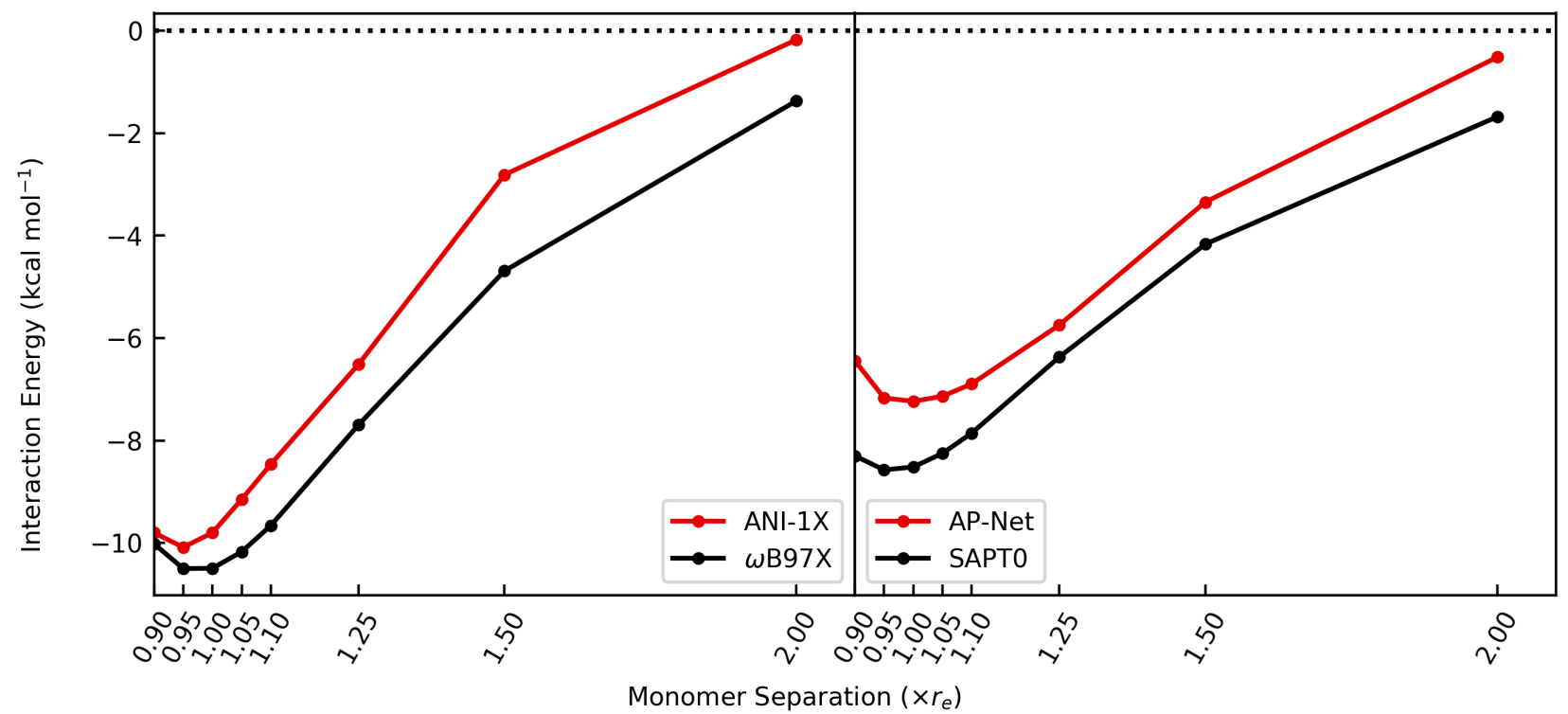

FIG. S5. ANI-1X and AP-Net are evaluated on the 8 geometries of the WaterPeptide dimer in the S66x 8 benchmark. Each ML model is compared to the level of theory used for parameterization: $\omega \mathrm{B} 97 \mathrm{X} / 6-31 \mathrm{G}^{*}$ for ANI-1X and SAPT0/jun-cc-pVDZ for AP-Net.

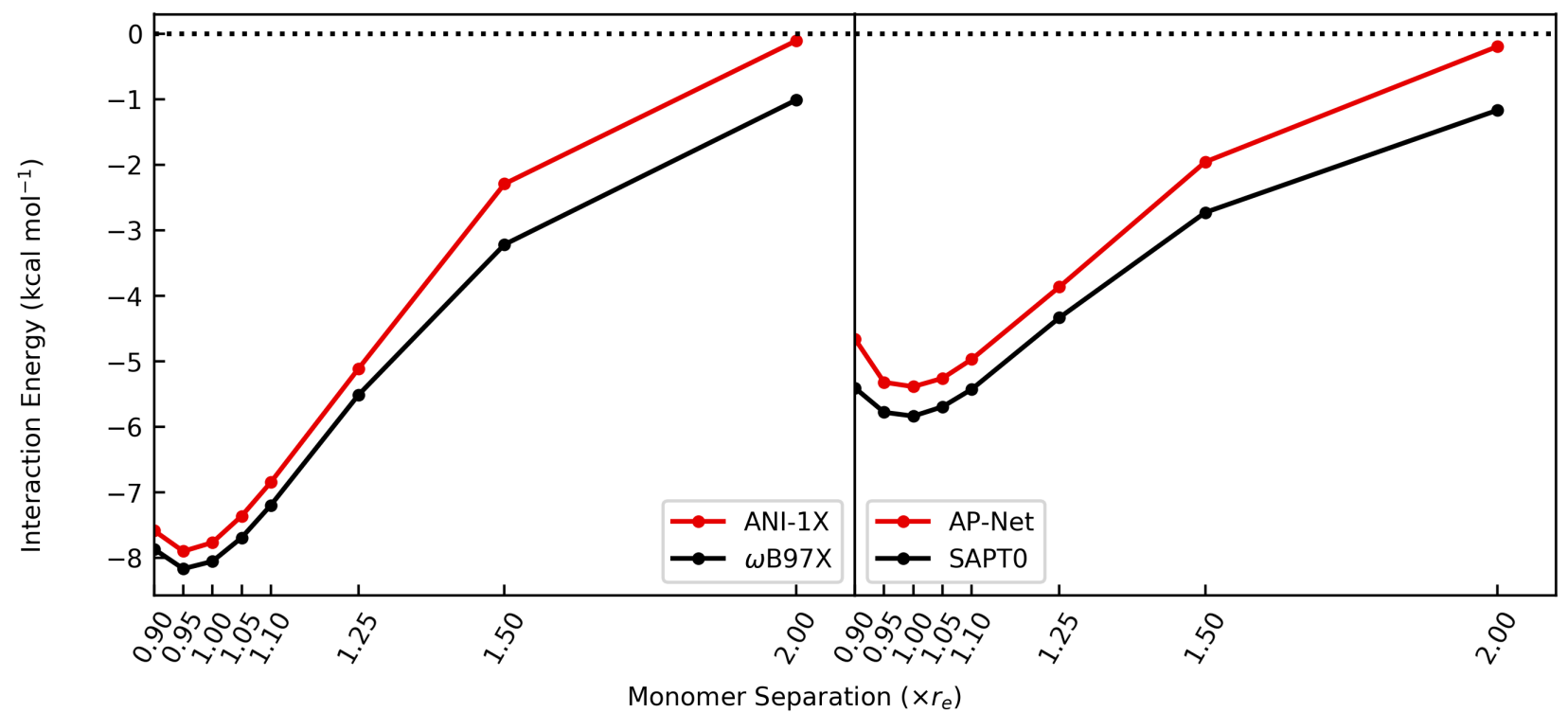

FIG. S6. ANI-1X and AP-Net are evaluated on the 8 geometries of the $\mathrm{MeOHMeOH}$ dimer in the S66x 8 benchmark. Each ML model is compared to the level of theory used for parameterization: $\omega \mathrm{B} 97 \mathrm{X} / 6-31 \mathrm{G}^{*}$ for ANI-1X and SAPT0/jun-cc-pVDZ for AP-Net. 


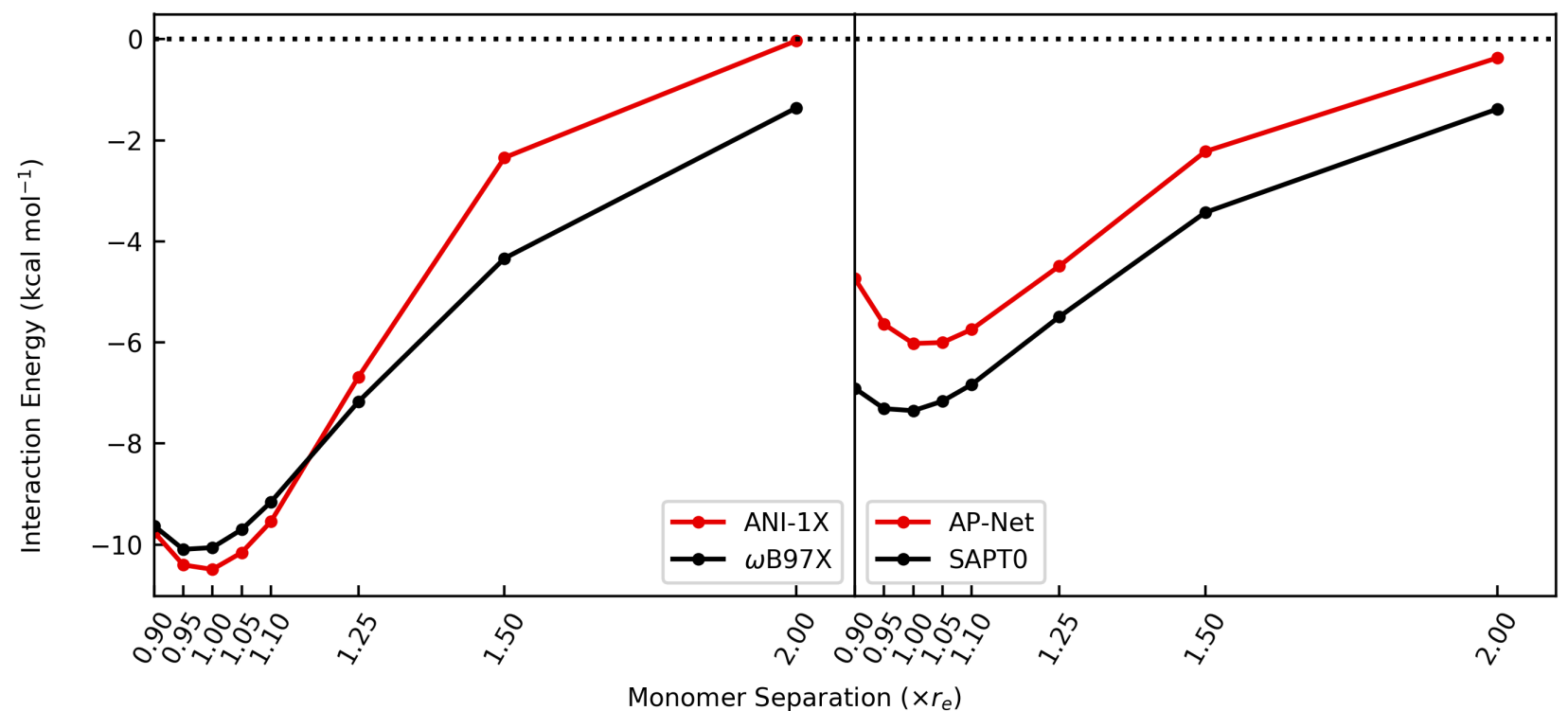

FIG. S7. ANI-1X and AP-Net are evaluated on the 8 geometries of the MeOHMeNH2 dimer in the S66x 8 benchmark. Each ML model is compared to the level of theory used for parameterization: $\omega \mathrm{B} 97 \mathrm{X} / 6-31 \mathrm{G}^{*}$ for ANI-1X and SAPT0/jun-cc-pVDZ for AP-Net.

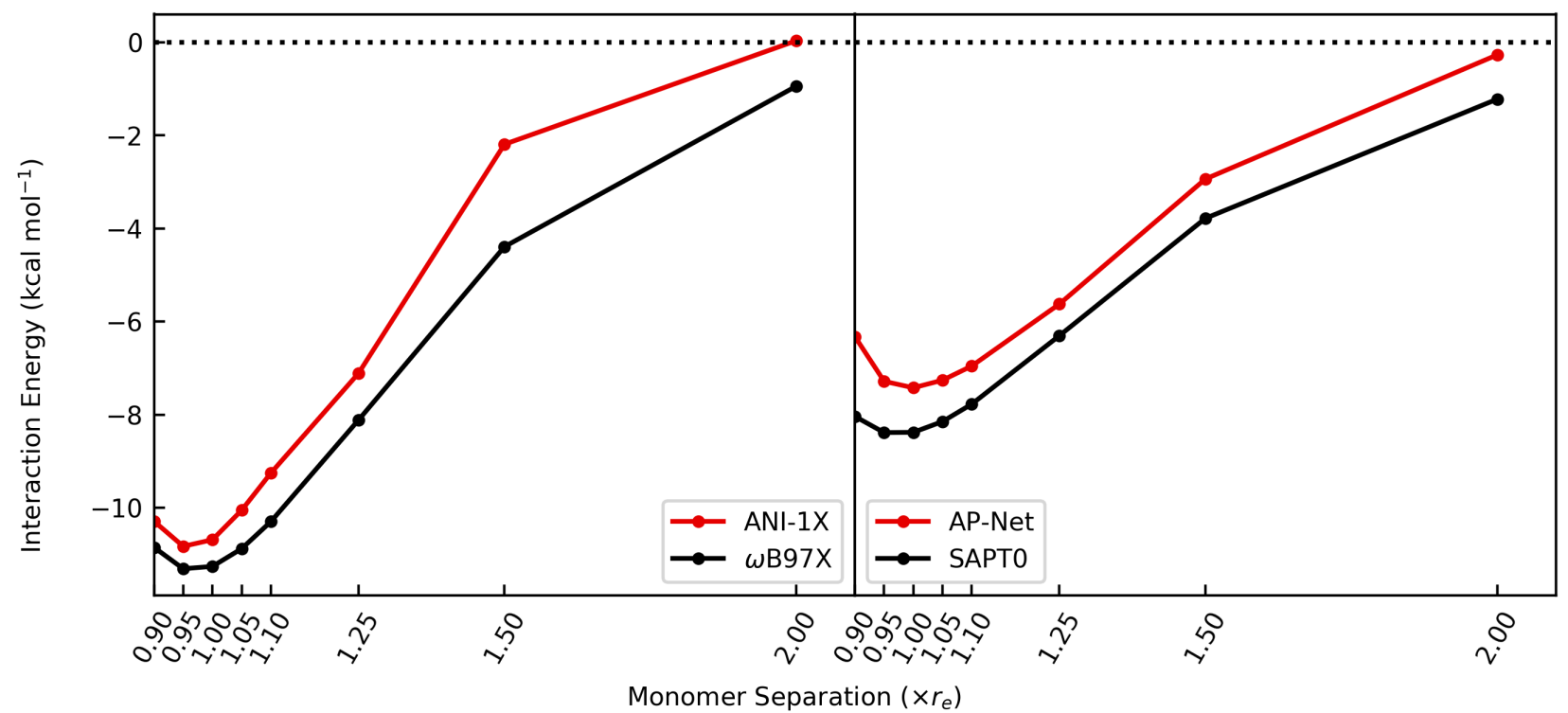

FIG. S8. ANI-1X and AP-Net are evaluated on the 8 geometries of the MeOHPeptide dimer in the S66x 8 benchmark. Each ML model is compared to the level of theory used for parameterization: $\omega \mathrm{B} 97 \mathrm{X} / 6-31 \mathrm{G}^{*}$ for ANI-1X and SAPT0/jun-cc-pVDZ for AP-Net. 


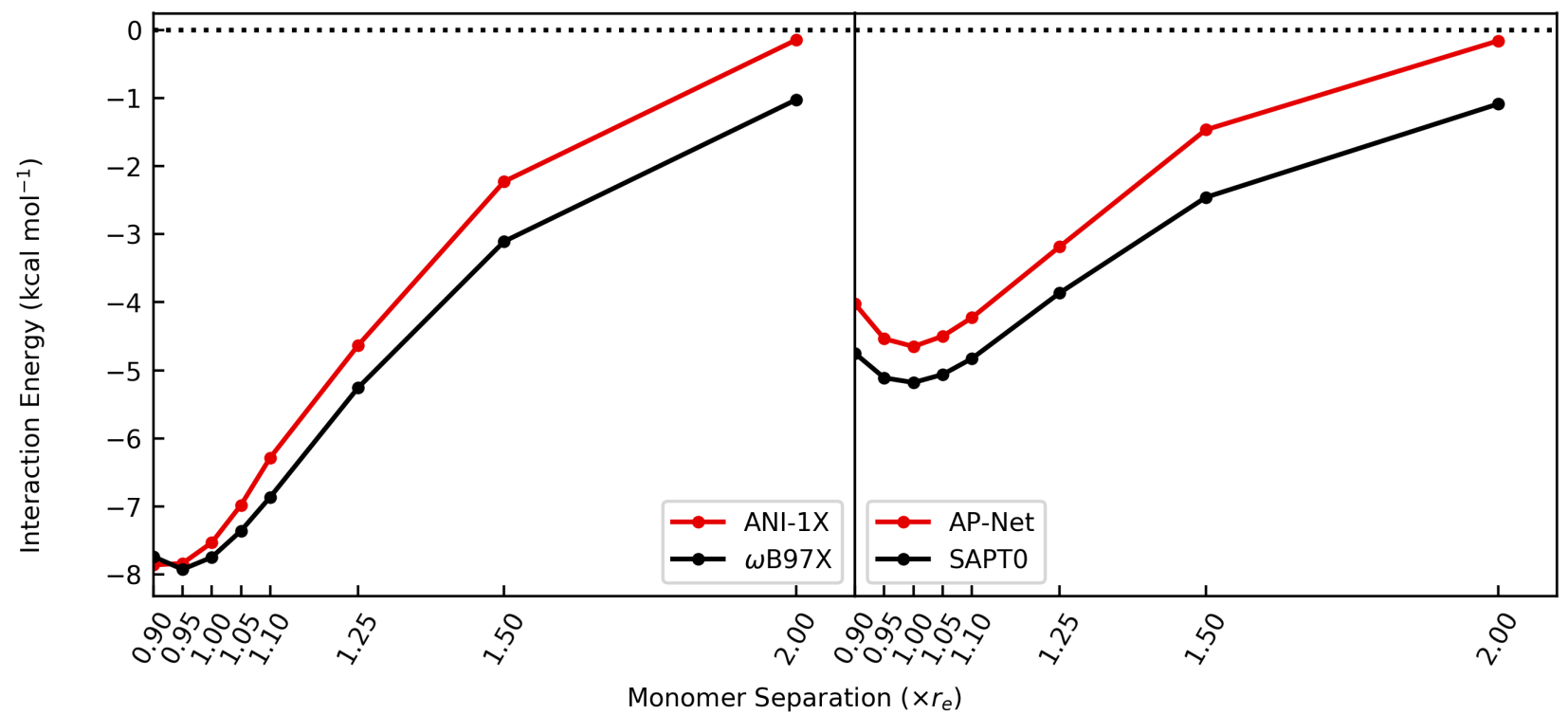

FIG. S9. ANI-1X and AP-Net are evaluated on the 8 geometries of the MeOHWater dimer in the S66x 8 benchmark. Each ML model is compared to the level of theory used for parameterization: $\omega \mathrm{B} 97 \mathrm{X} / 6-31 \mathrm{G}^{*}$ for ANI-1X and SAPT0/jun-cc-pVDZ for AP-Net.

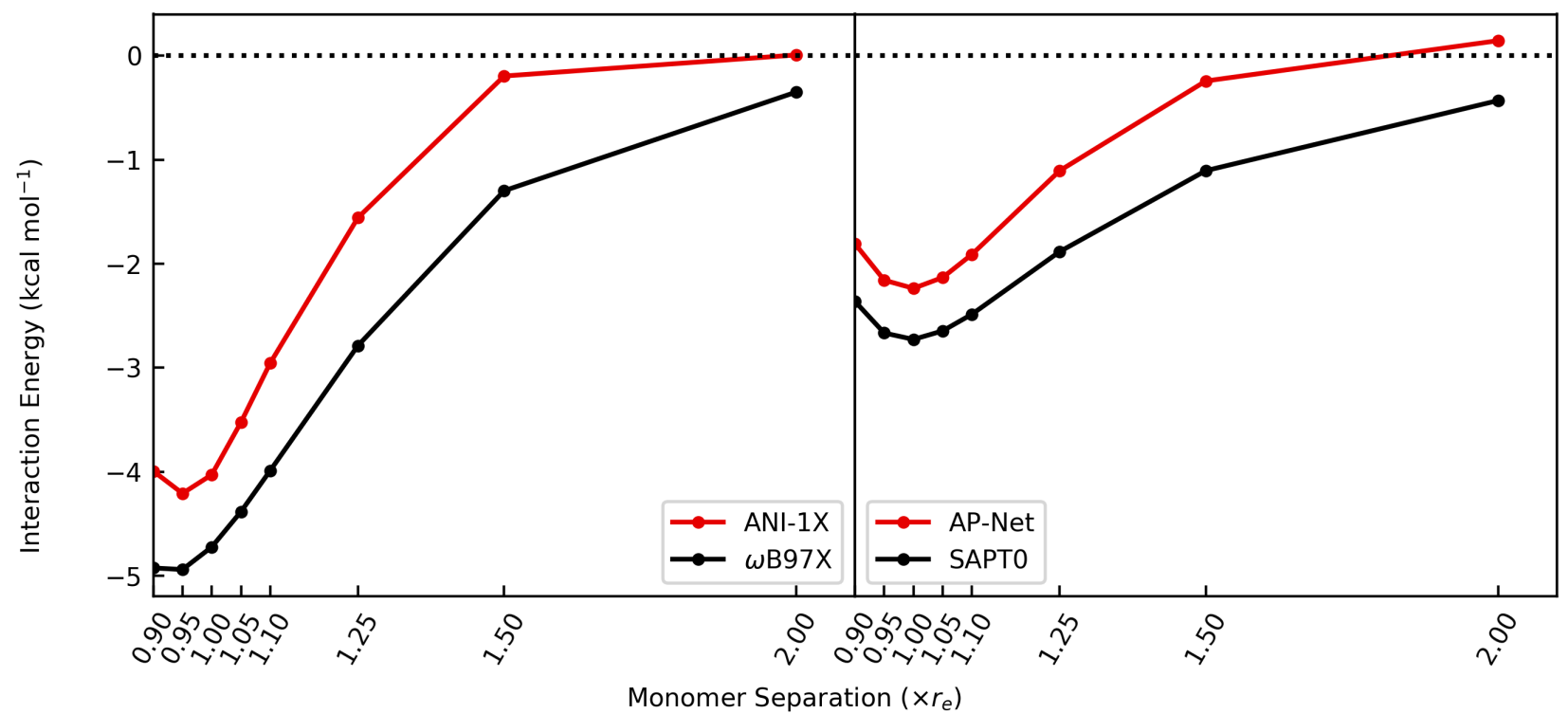

FIG. S10. ANI-1X and AP-Net are evaluated on the 8 geometries of the $M e N H 2 M e O H$ dimer in the S66x 8 benchmark. Each ML model is compared to the level of theory used for parameterization: $\omega \mathrm{B} 97 \mathrm{X} / 6-31 \mathrm{G}^{*}$ for ANI-1X and SAPT0/jun-cc-pVDZ for AP-Net. 


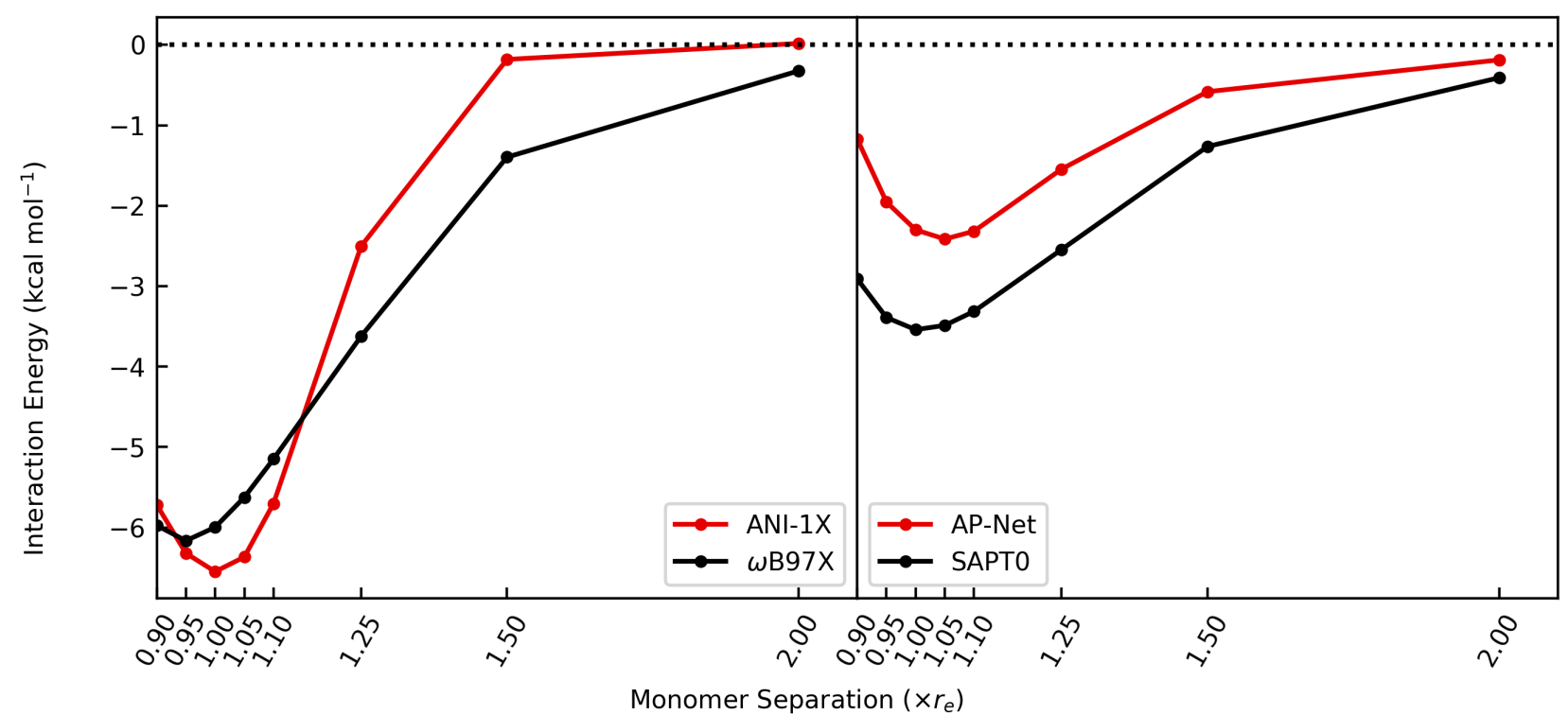

FIG. S11. ANI-1X and AP-Net are evaluated on the 8 geometries of the MeNH2MeNH2 dimer in the S66x 8 benchmark. Each ML model is compared to the level of theory used for parameterization: $\omega \mathrm{B} 97 \mathrm{X} / 6-31 \mathrm{G}^{*}$ for ANI-1X and SAPT0/jun-cc-pVDZ for AP-Net.

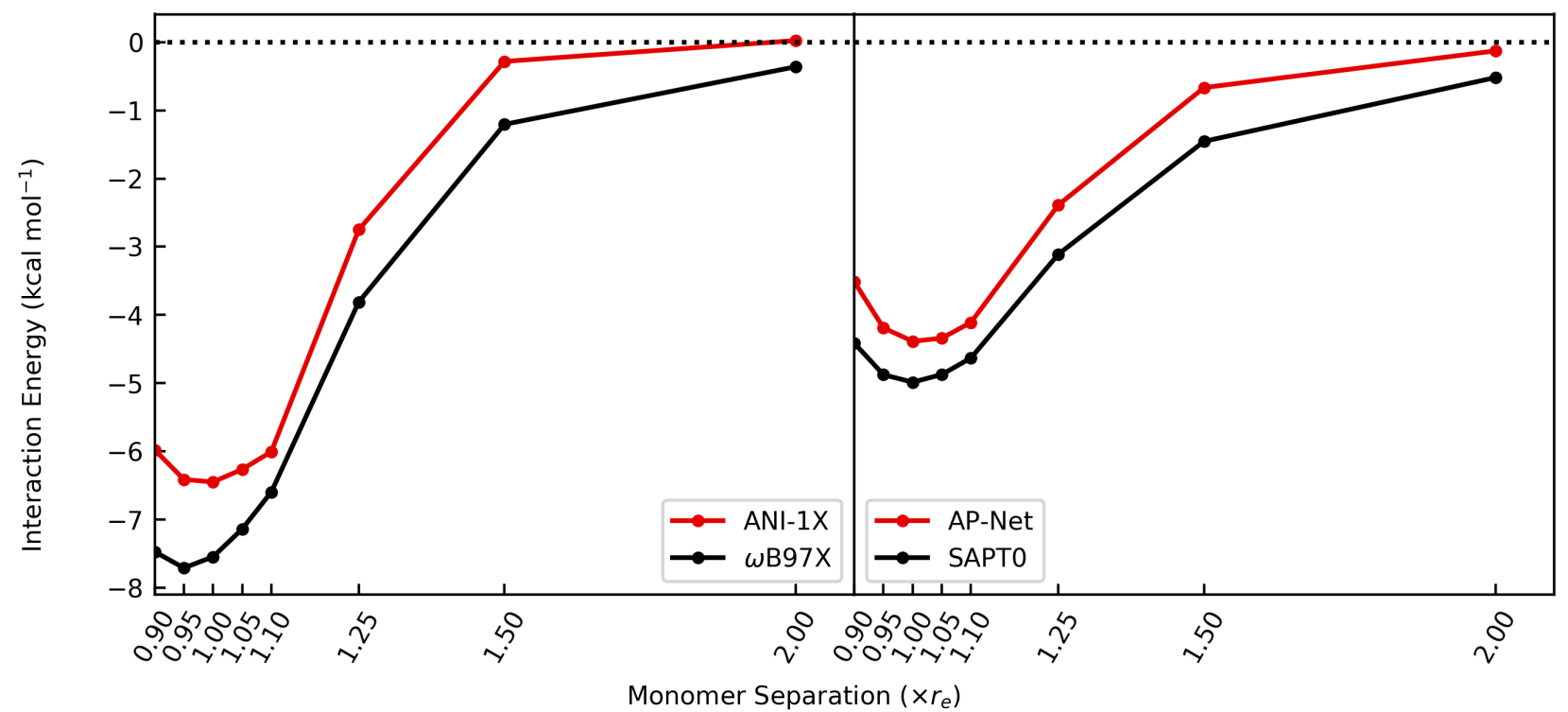

FIG. S12. ANI-1X and AP-Net are evaluated on the 8 geometries of the MeNH2Peptide dimer in the S66x8 benchmark. Each ML model is compared to the level of theory used for parameterization: $\omega \mathrm{B} 97 \mathrm{X} / 6-31 \mathrm{G}^{*}$ for ANI-1X and SAPT0/jun-cc-pVDZ for AP-Net. 


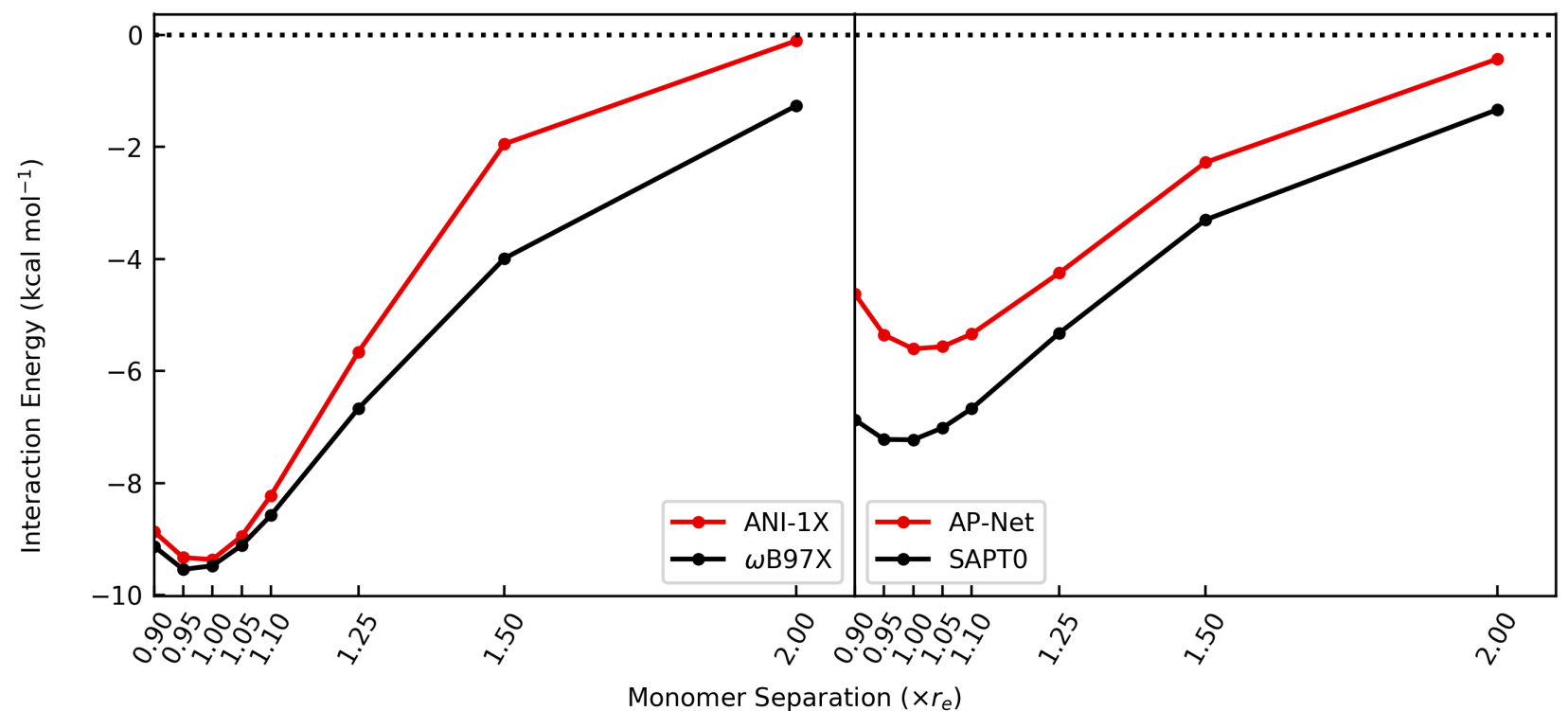

FIG. S13. ANI-1X and AP-Net are evaluated on the 8 geometries of the MeNH2Water dimer in the S66x 8 benchmark. Each ML model is compared to the level of theory used for parameterization: $\omega \mathrm{B} 97 \mathrm{X} / 6-31 \mathrm{G}^{*}$ for ANI-1X and SAPT0/jun-cc-pVDZ for AP-Net.

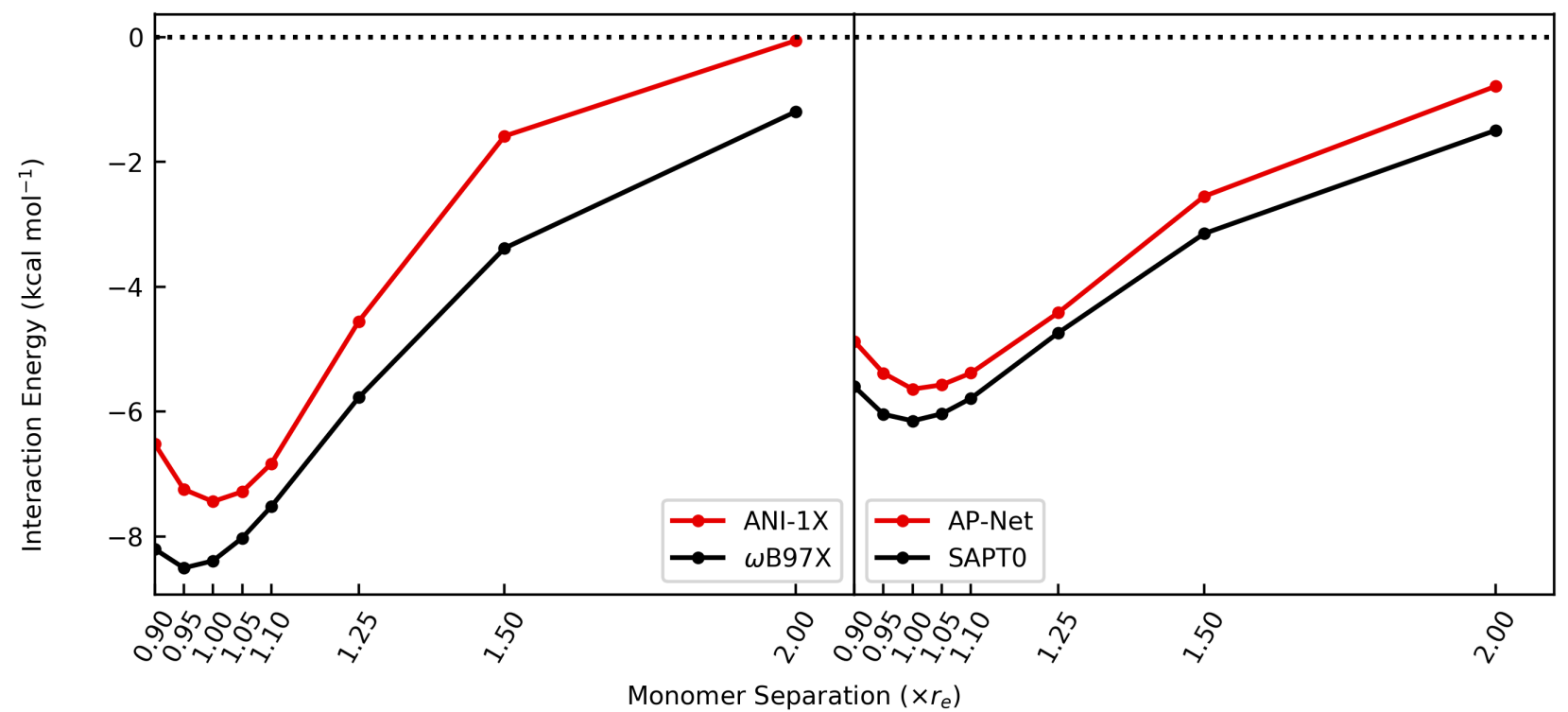

FIG. S14. ANI-1X and AP-Net are evaluated on the 8 geometries of the PeptideMeOH dimer in the S66x8 benchmark. Each ML model is compared to the level of theory used for parameterization: $\omega \mathrm{B} 97 \mathrm{X} / 6-31 \mathrm{G}^{*}$ for ANI-1X and SAPT0/jun-cc-pVDZ for AP-Net. 


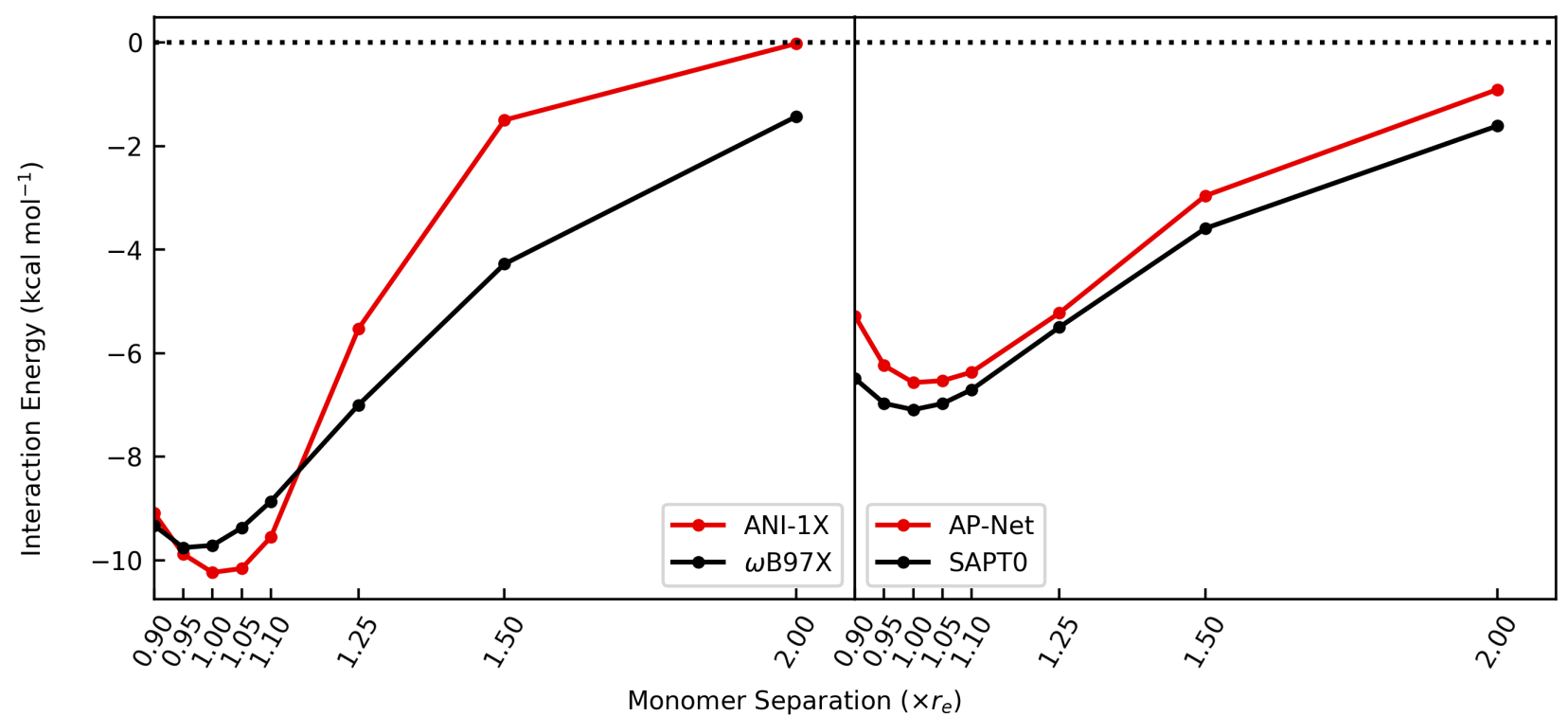

FIG. S15. ANI-1X and AP-Net are evaluated on the 8 geometries of the PeptideMeNH2 dimer in the S66x 8 benchmark. Each ML model is compared to the level of theory used for parameterization: $\omega \mathrm{B} 97 \mathrm{X} / 6-31 \mathrm{G}^{*}$ for ANI-1X and SAPT0/jun-cc-pVDZ for AP-Net.

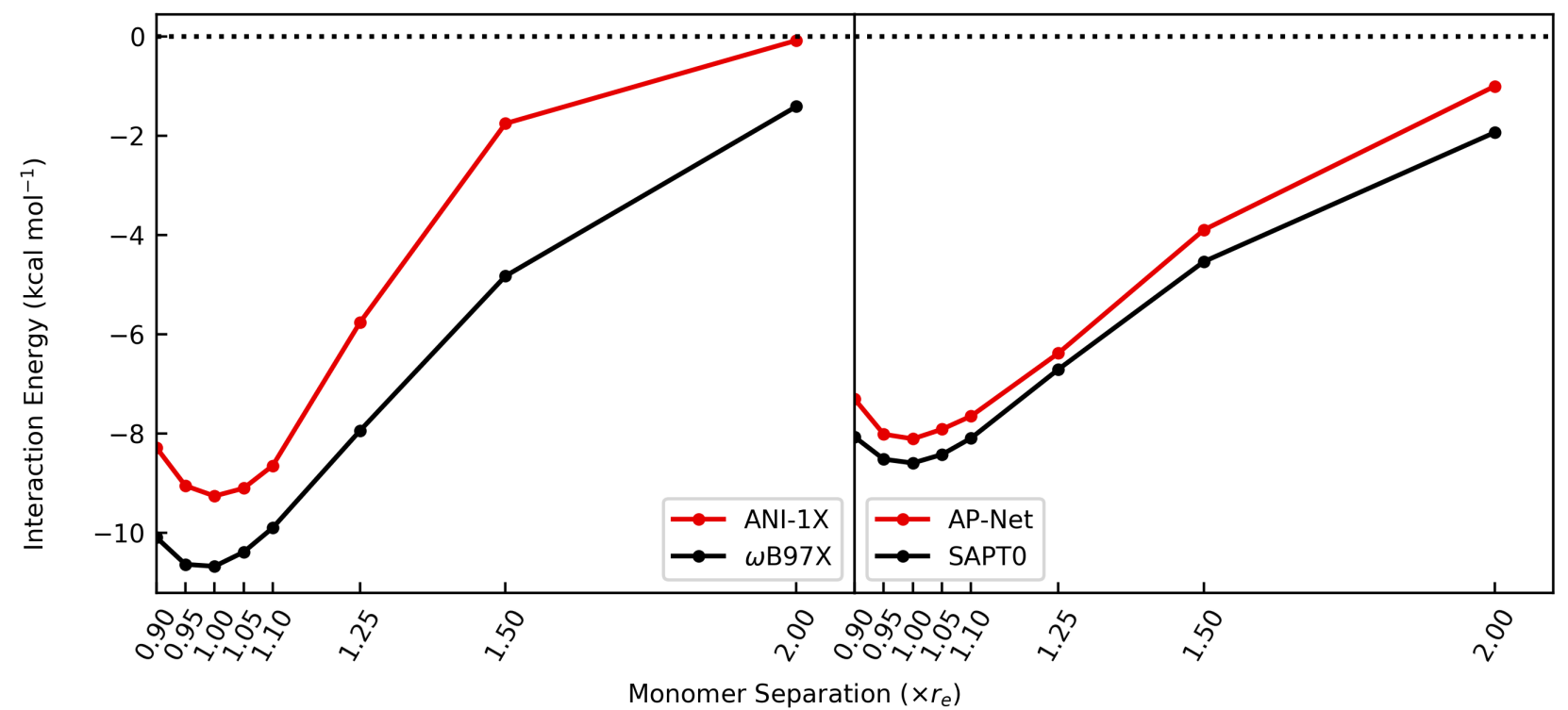

FIG. S16. ANI-1X and AP-Net are evaluated on the 8 geometries of the PeptidePeptide dimer in the S66x8 benchmark. Each ML model is compared to the level of theory used for parameterization: $\omega \mathrm{B} 97 \mathrm{X} / 6-31 \mathrm{G}^{*}$ for ANI-1X and SAPT0/jun-cc-pVDZ for AP-Net. 


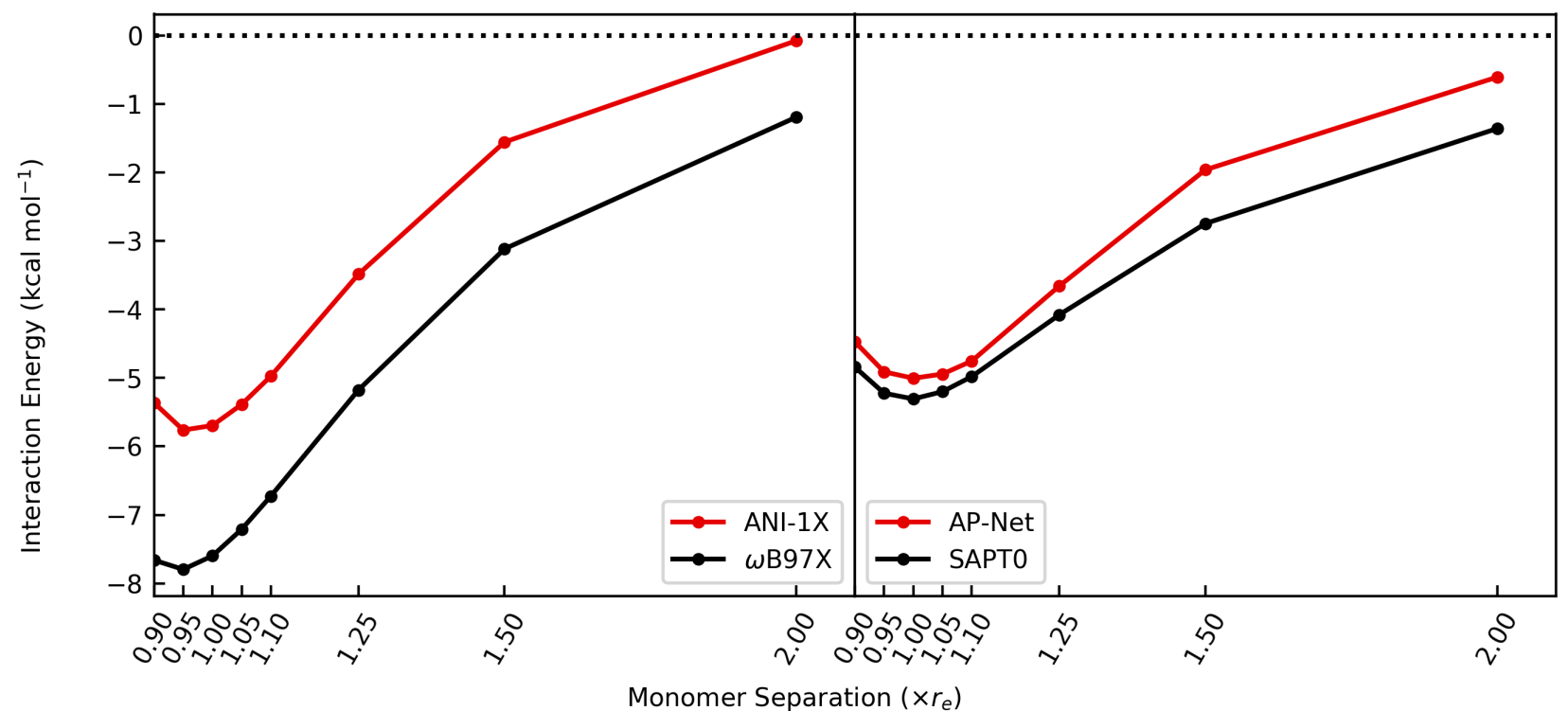

FIG. S17. ANI-1X and AP-Net are evaluated on the 8 geometries of the PeptideWater dimer in the S66x 8 benchmark. Each ML model is compared to the level of theory used for parameterization: $\omega \mathrm{B} 97 \mathrm{X} / 6-31 \mathrm{G}^{*}$ for ANI-1X and SAPT0/jun-cc-pVDZ for AP-Net.

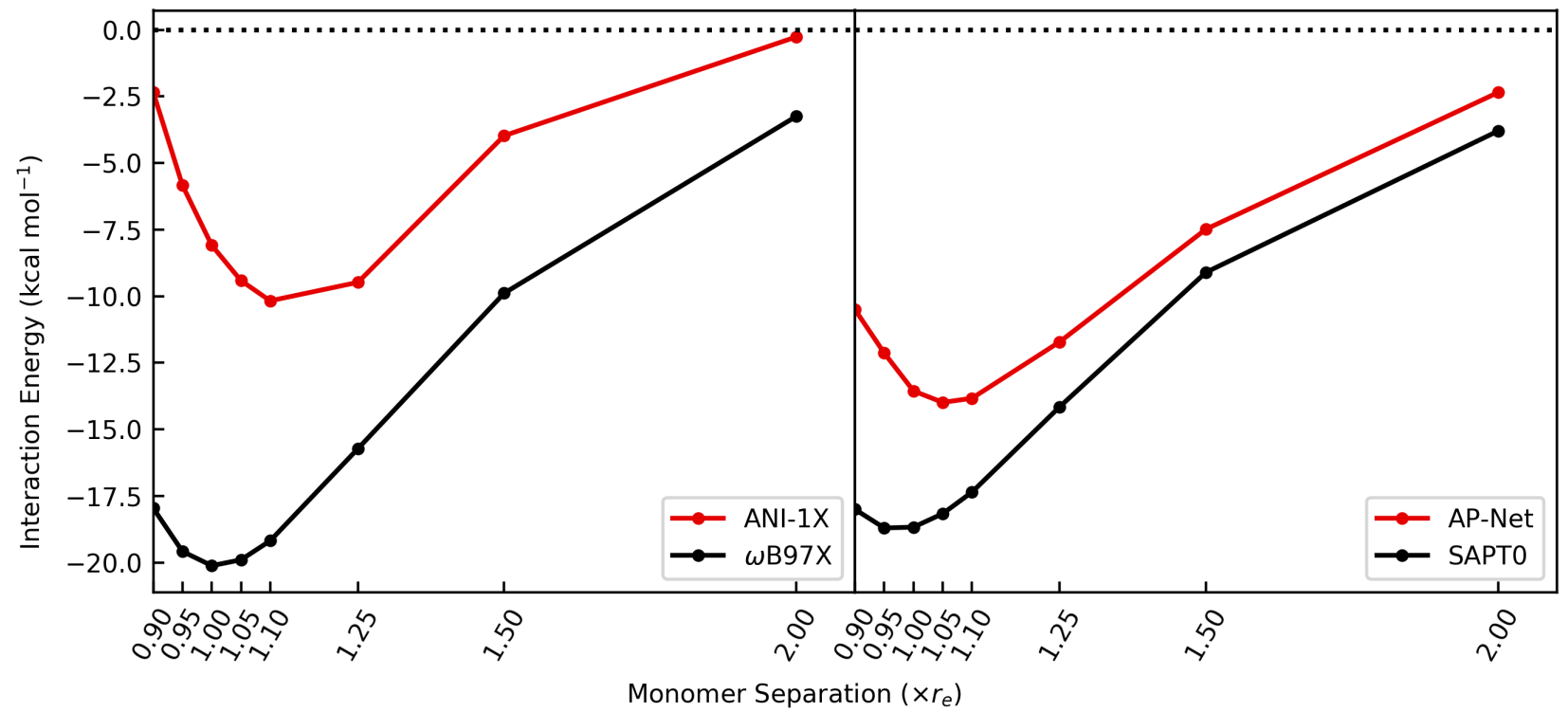

FIG. S18. ANI-1X and AP-Net are evaluated on the 8 geometries of the UracilUracilBP dimer in the S66x 8 benchmark. Each ML model is compared to the level of theory used for parameterization: $\omega \mathrm{B} 97 \mathrm{X} / 6-31 \mathrm{G}^{*}$ for ANI-1X and SAPT0/jun-cc-pVDZ for AP-Net. 


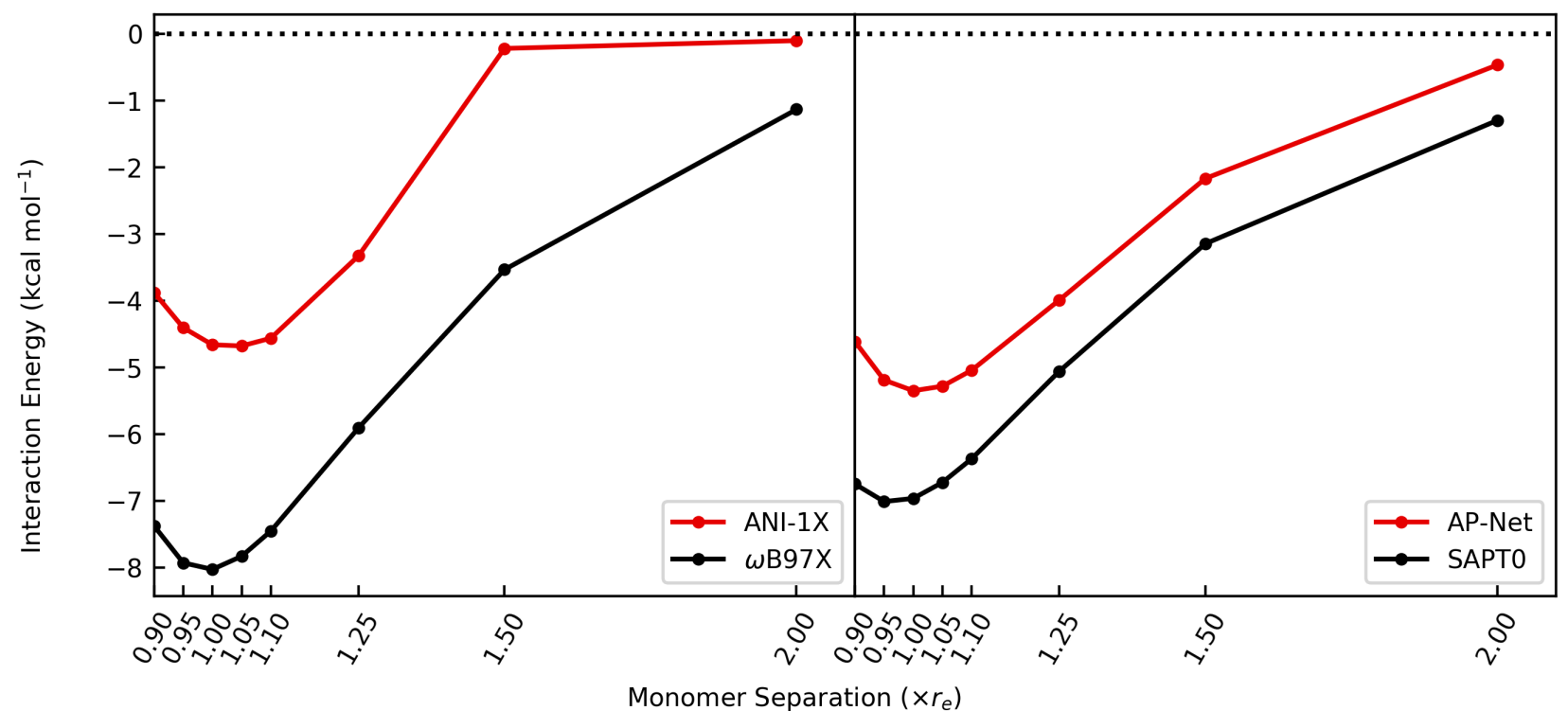

FIG. S19. ANI-1X and AP-Net are evaluated on the 8 geometries of the WaterPyridine dimer in the S66x 8 benchmark. Each ML model is compared to the level of theory used for parameterization: $\omega \mathrm{B} 97 \mathrm{X} / 6-31 \mathrm{G}^{*}$ for ANI-1X and SAPT0/jun-cc-pVDZ for AP-Net.

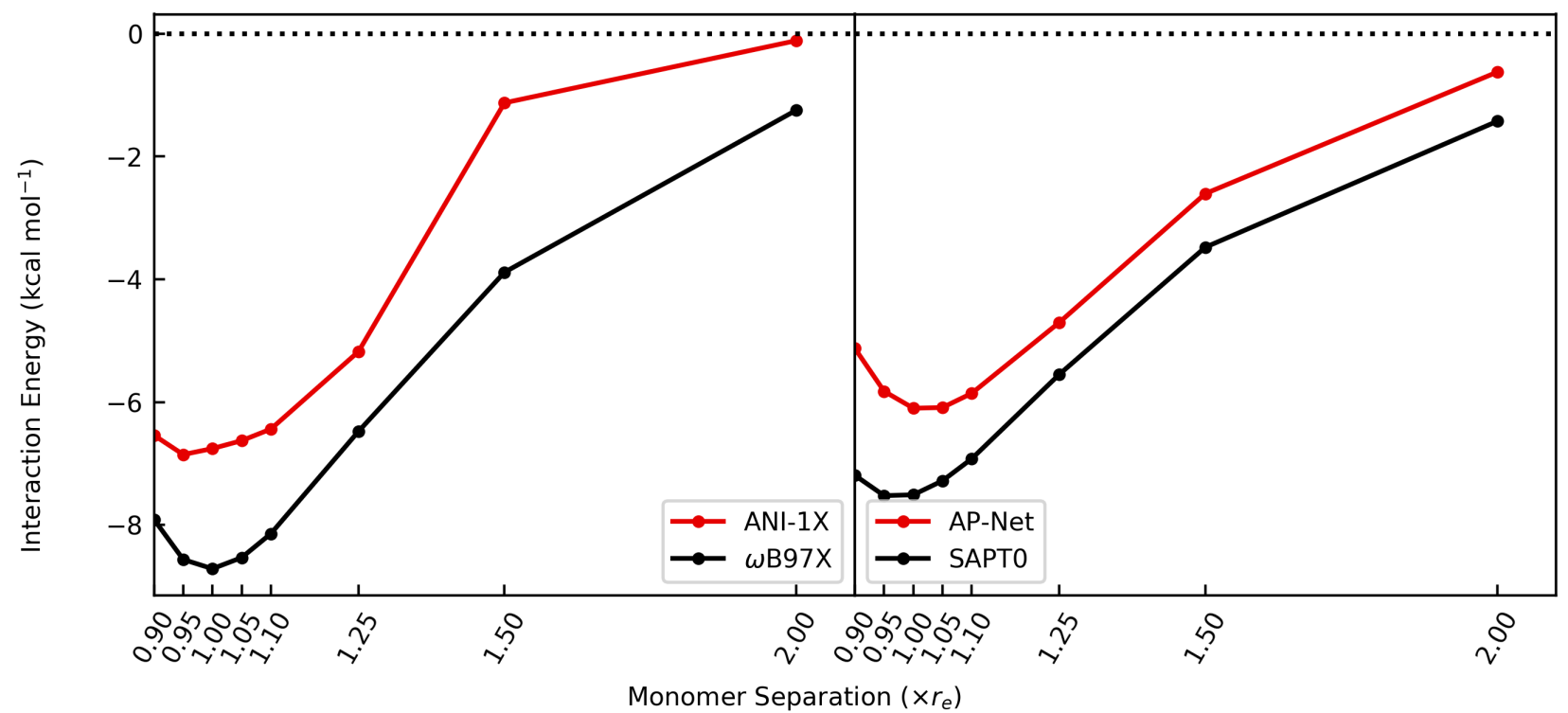

FIG. S20. ANI-1X and AP-Net are evaluated on the 8 geometries of the MeOHPyridine dimer in the S66x 8 benchmark. Each ML model is compared to the level of theory used for parameterization: $\omega \mathrm{B} 97 \mathrm{X} / 6-31 \mathrm{G}^{*}$ for ANI-1X and SAPT0/jun-cc-pVDZ for AP-Net. 


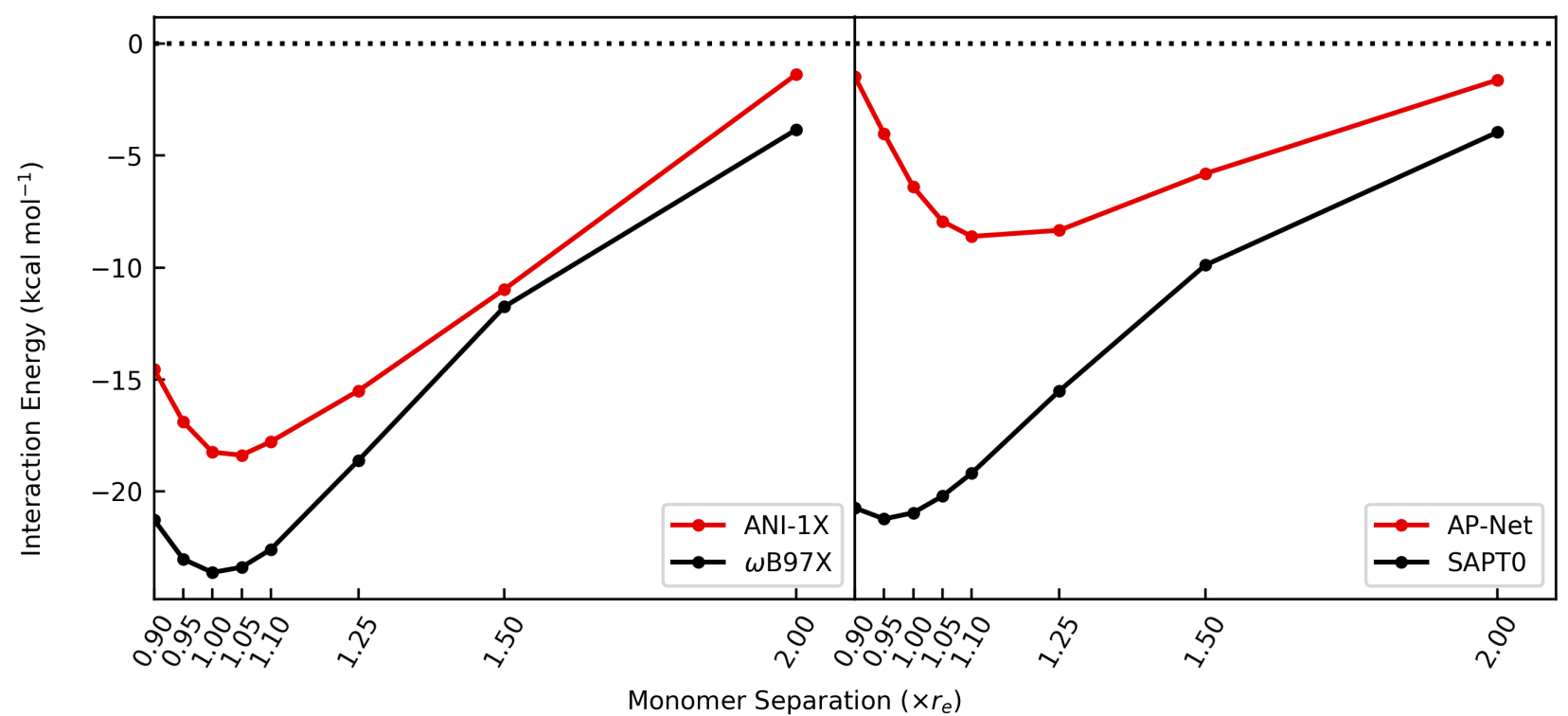

FIG. S21. ANI-1X and AP-Net are evaluated on the 8 geometries of the AcOHAcOH dimer in the S66x8 benchmark. Each ML model is compared to the level of theory used for parameterization: $\omega \mathrm{B} 97 \mathrm{X} / 6-31 \mathrm{G}^{*}$ for ANI-1X and SAPT0/jun-cc-pVDZ for AP-Net.

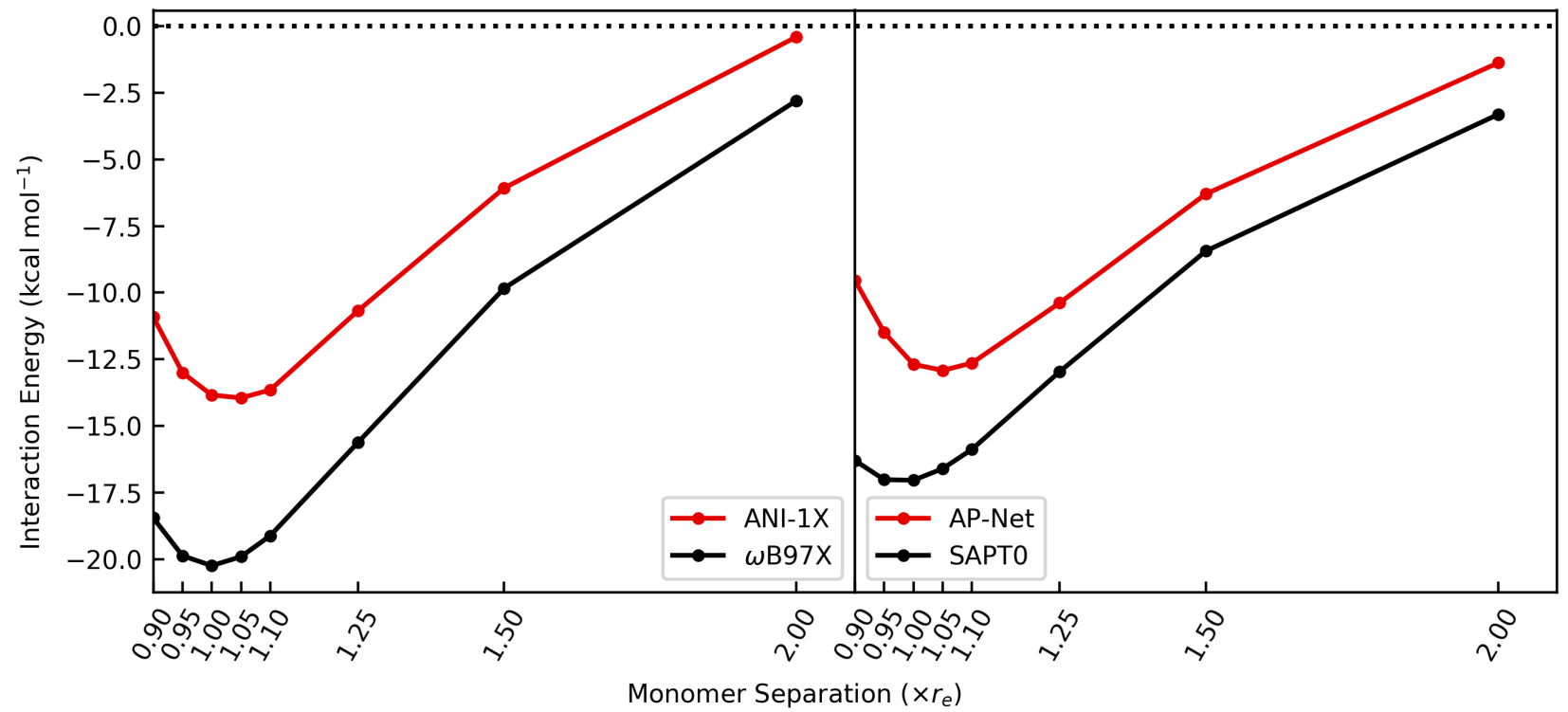

FIG. S22. ANI-1X and AP-Net are evaluated on the 8 geometries of the AcNH2AcNH2 dimer in the S66x 8 benchmark. Each ML model is compared to the level of theory used for parameterization: $\omega \mathrm{B} 97 \mathrm{X} / 6-31 \mathrm{G}^{*}$ for ANI-1X and SAPT0/jun-cc-pVDZ for AP-Net. 


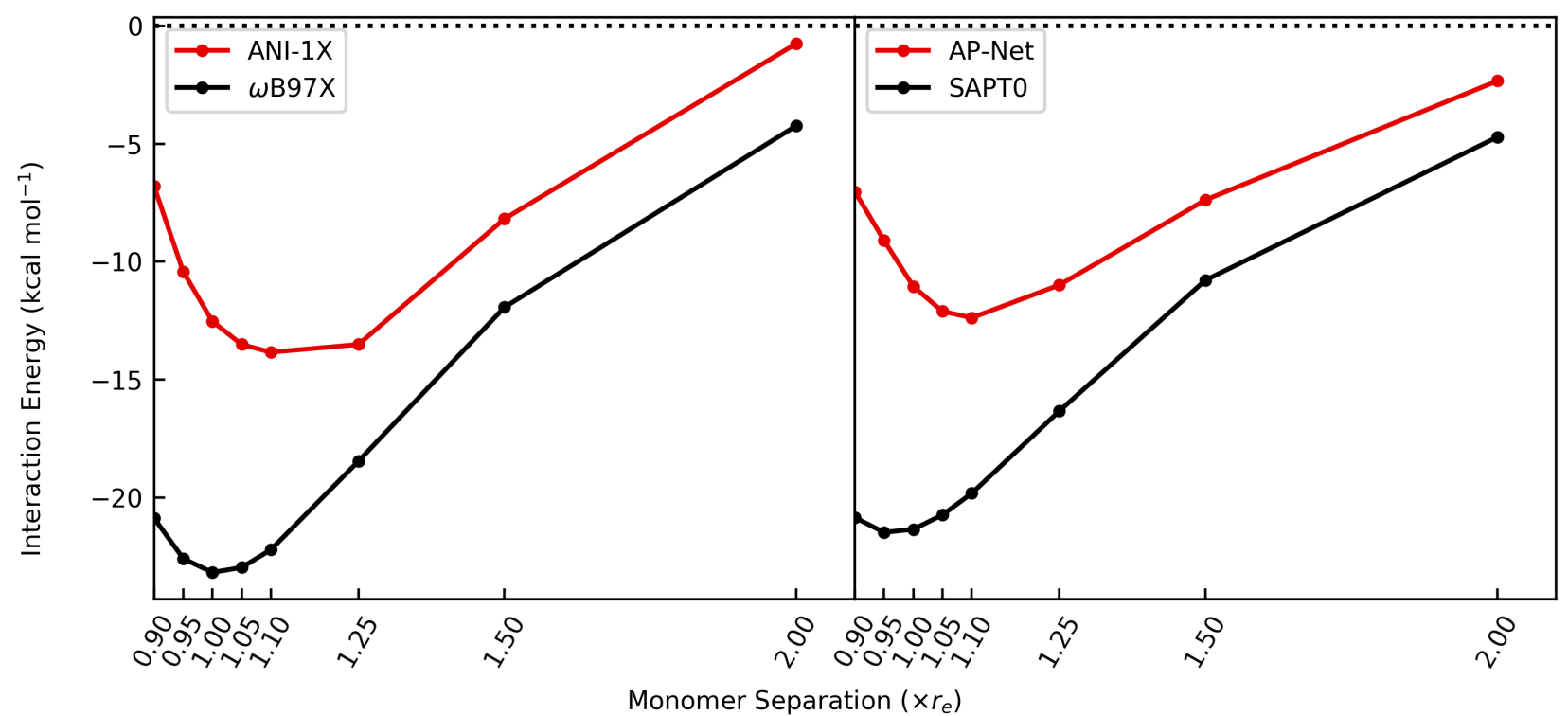

FIG. S23. ANI-1X and AP-Net are evaluated on the 8 geometries of the AcOHUracil dimer in the S66x 8 benchmark. Each ML model is compared to the level of theory used for parameterization: $\omega \mathrm{B} 97 \mathrm{X} / 6-31 \mathrm{G}^{*}$ for ANI-1X and SAPT0/jun-cc-pVDZ for AP-Net.

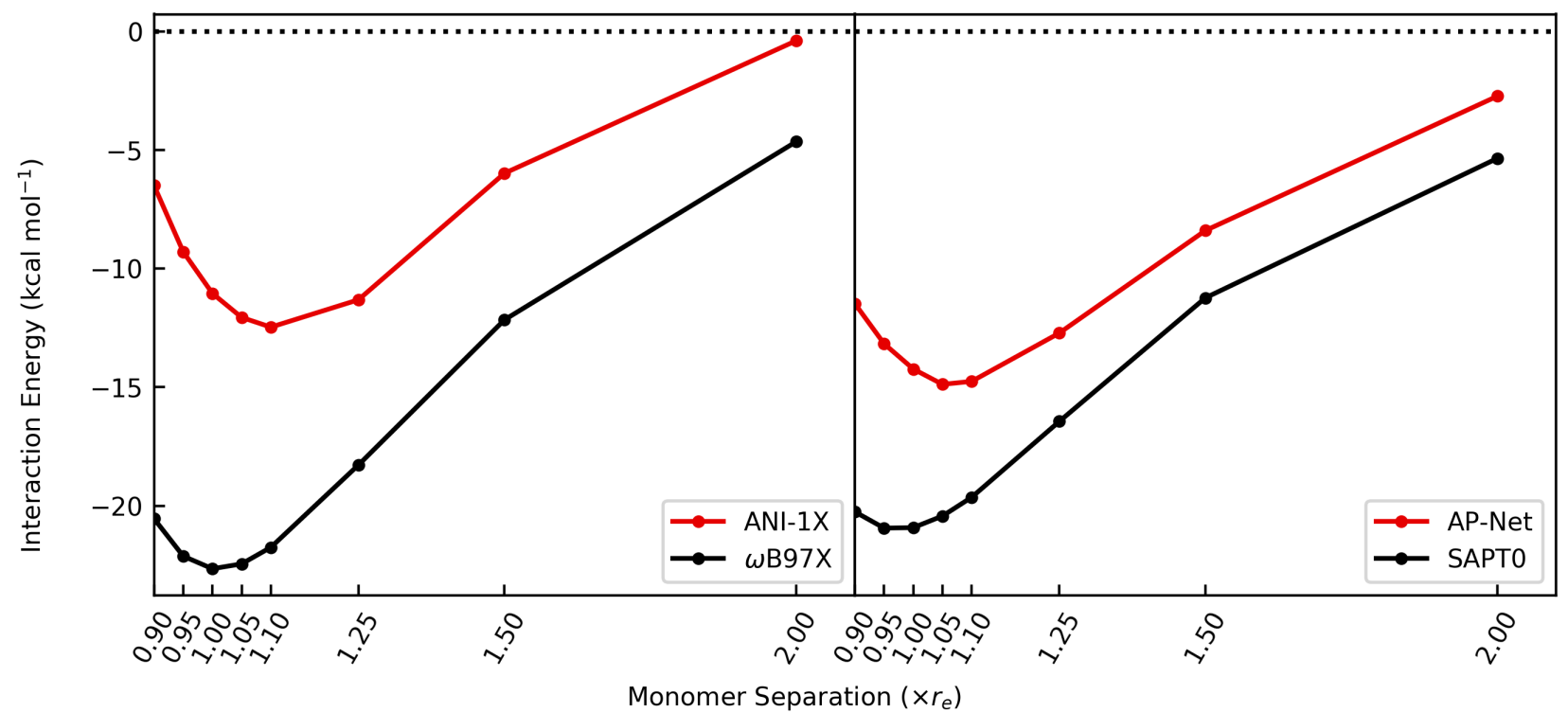

FIG. S24. ANI-1X and AP-Net are evaluated on the 8 geometries of the AcNH2Uracil dimer in the S66x 8 benchmark. Each ML model is compared to the level of theory used for parameterization: $\omega \mathrm{B} 97 \mathrm{X} / 6-31 \mathrm{G}^{*}$ for ANI-1X and SAPT0/jun-cc-pVDZ for AP-Net. 


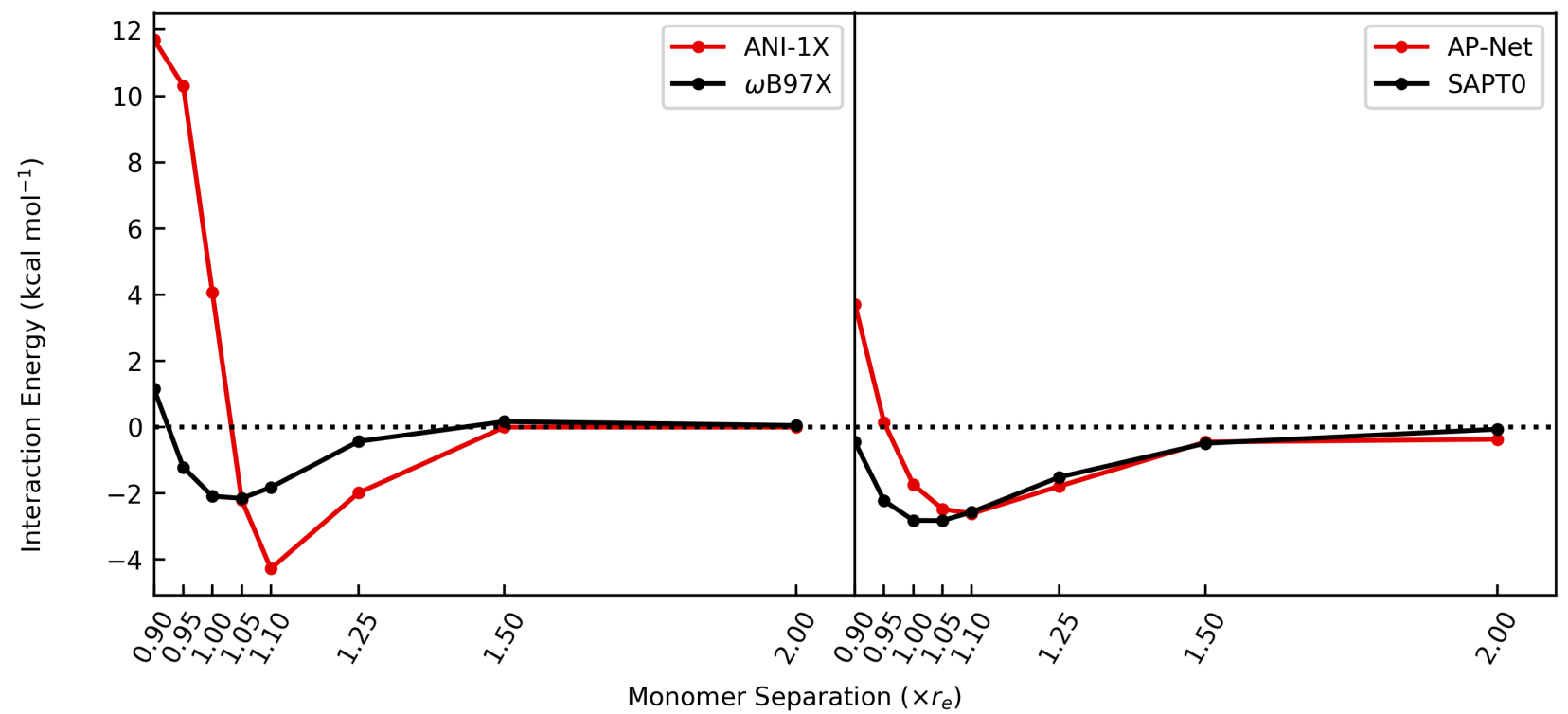

FIG. S25. ANI-1X and AP-Net are evaluated on the 8 geometries of the BenzeneBenzenepipi dimer in the S66x8 benchmark. Each ML model is compared to the level of theory used for parameterization: $\omega$ B97X/631G* for ANI-1X and SAPT0/jun-cc-pVDZ for AP-Net.

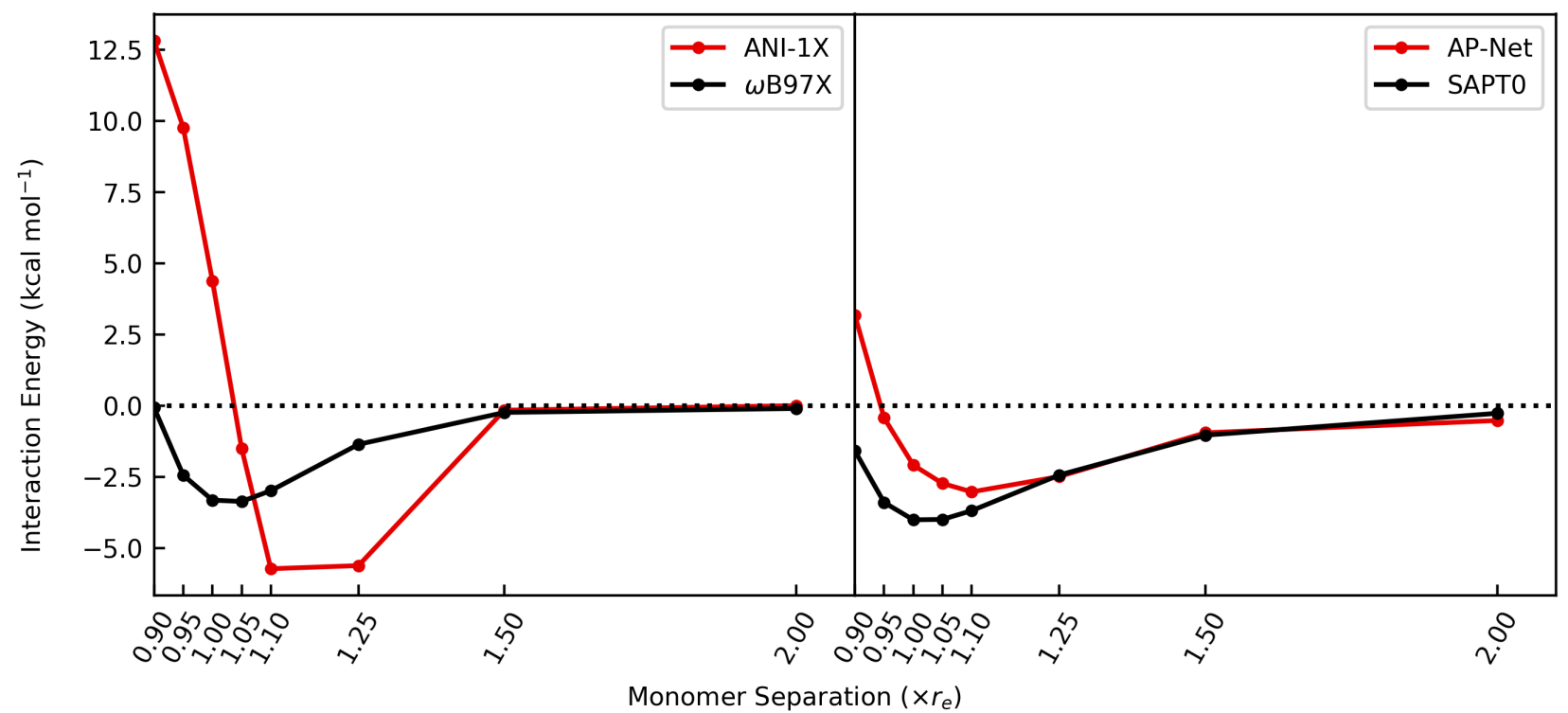

FIG. S26. ANI-1X and AP-Net are evaluated on the 8 geometries of the PyridinePyridinepipi dimer in the S66x8 benchmark. Each ML model is compared to the level of theory used for parameterization: $\omega$ B97X/631G* for ANI-1X and SAPT0/jun-cc-pVDZ for AP-Net. 


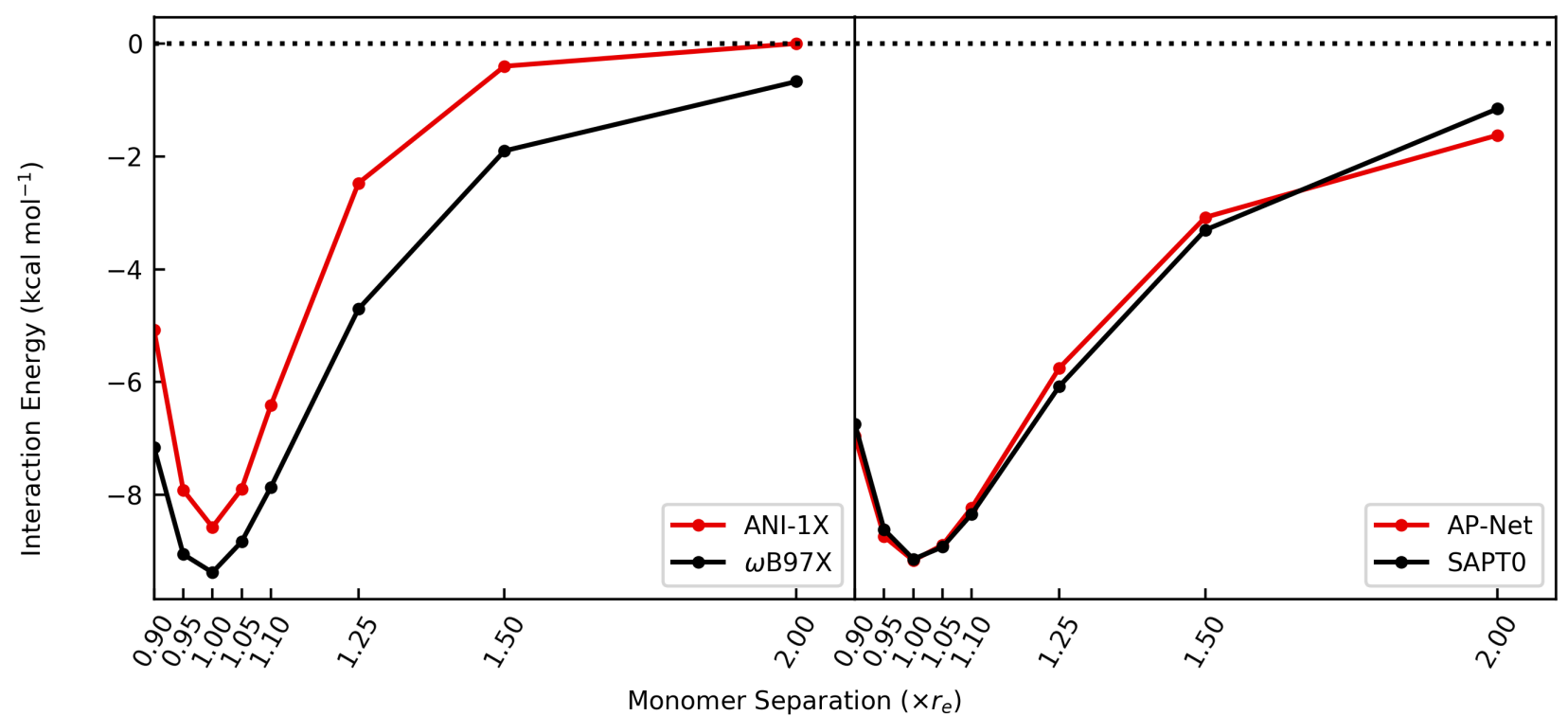

FIG. S27. ANI-1X and AP-Net are evaluated on the 8 geometries of the UracilUracilpipi dimer in the S66x 8 benchmark. Each ML model is compared to the level of theory used for parameterization: $\omega \mathrm{B} 97 \mathrm{X} / 6-31 \mathrm{G}^{*}$ for ANI-1X and SAPT0/jun-cc-pVDZ for AP-Net.

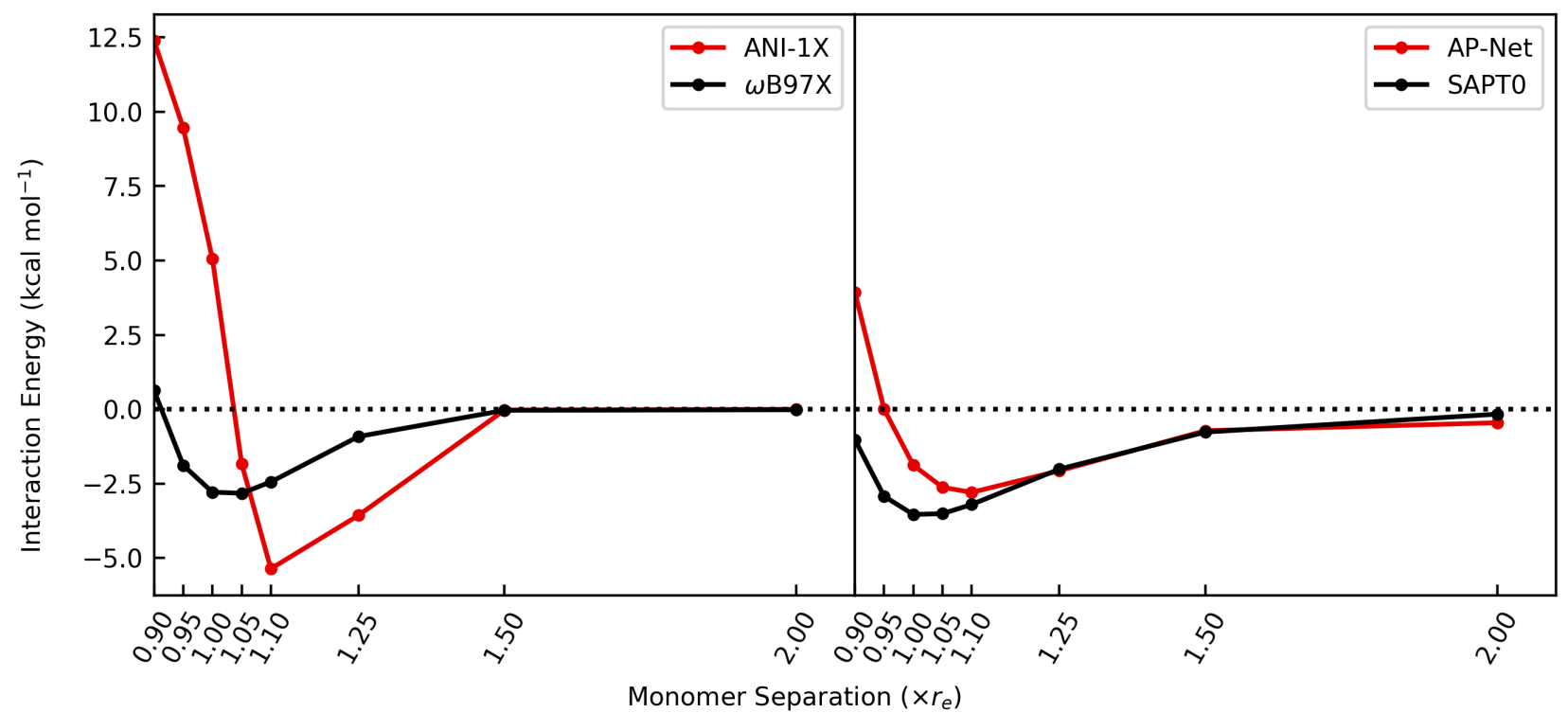

FIG. S28. ANI-1X and AP-Net are evaluated on the 8 geometries of the BenzenePyridinepipi dimer in the S66x8 benchmark. Each ML model is compared to the level of theory used for parameterization: $\omega$ B97X/631G* for ANI-1X and SAPT0/jun-cc-pVDZ for AP-Net. 


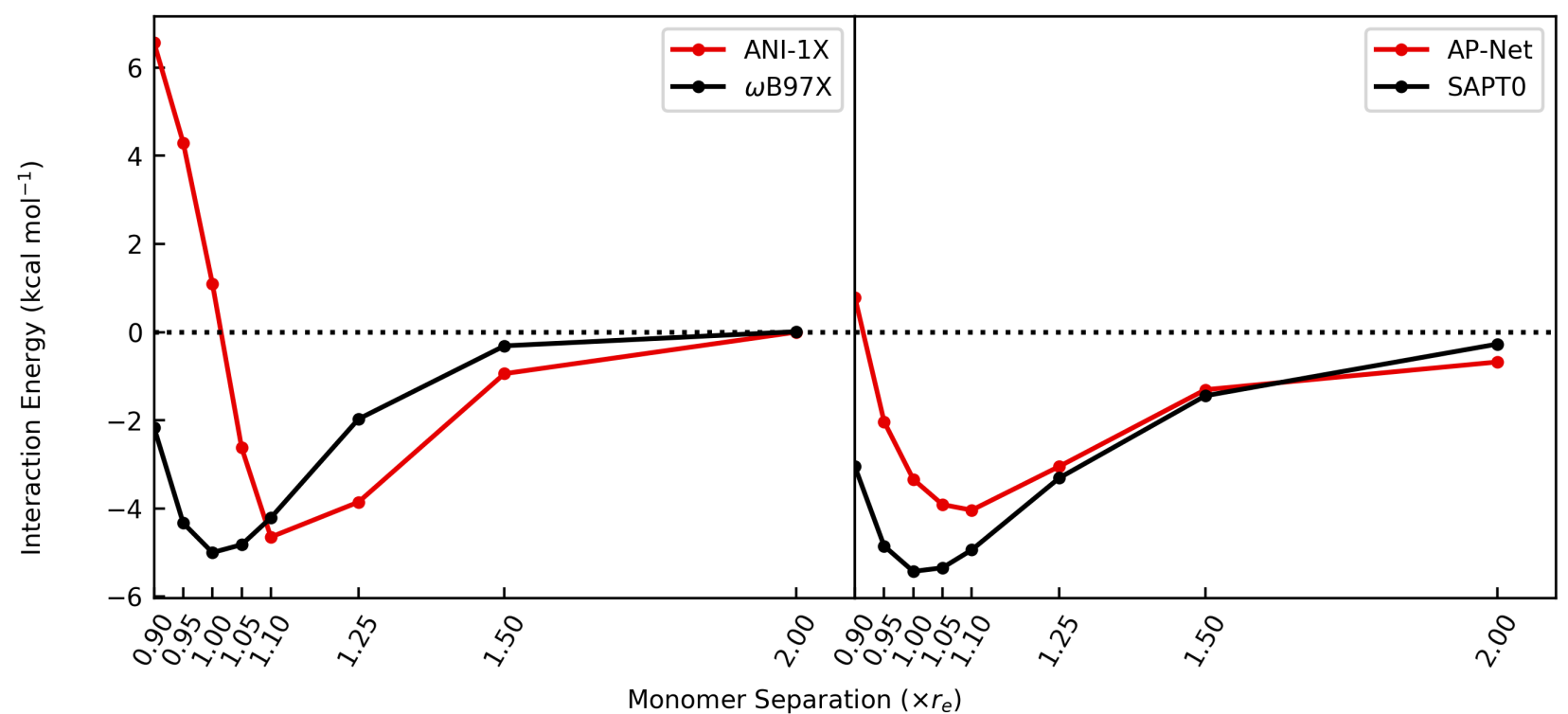

FIG. S29. ANI-1X and AP-Net are evaluated on the 8 geometries of the BenzeneUracilpipi dimer in the S66x8 benchmark. Each ML model is compared to the level of theory used for parameterization: $\omega$ B97X/631G* for ANI-1X and SAPT0/jun-cc-pVDZ for AP-Net.

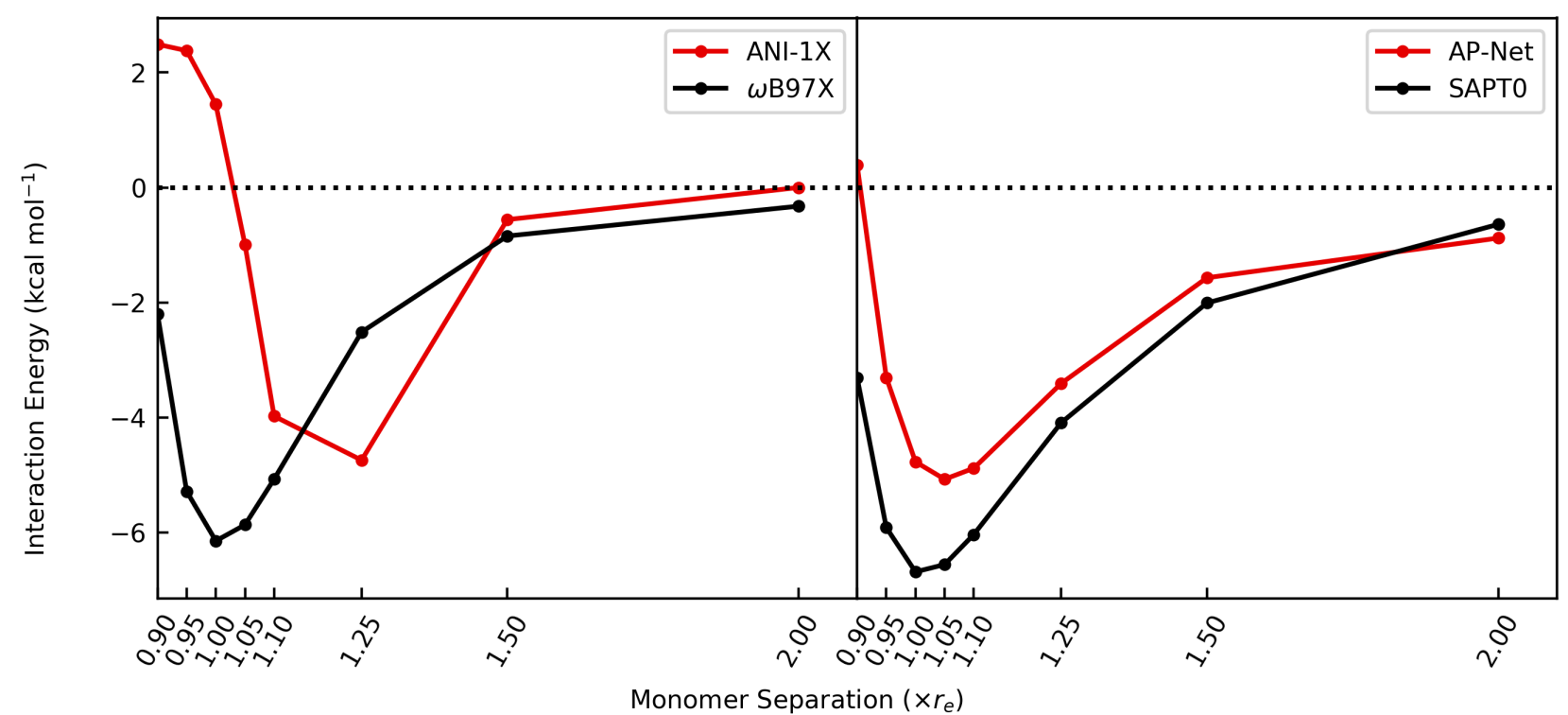

FIG. S30. ANI-1X and AP-Net are evaluated on the 8 geometries of the PyridineUracilpipi dimer in the S66x8 benchmark. Each ML model is compared to the level of theory used for parameterization: $\omega$ B97X/631G* for ANI-1X and SAPT0/jun-cc-pVDZ for AP-Net. 


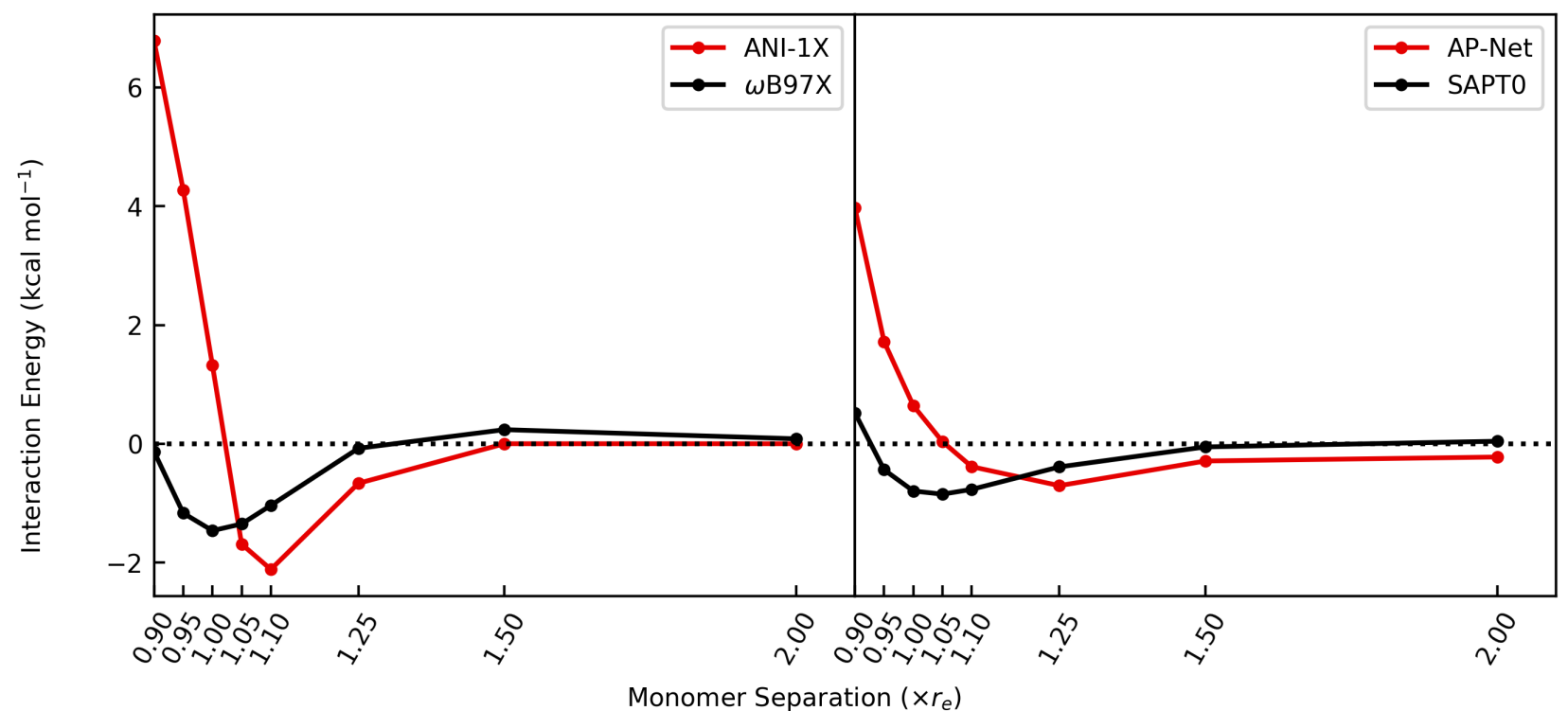

FIG. S31. ANI-1X and AP-Net are evaluated on the 8 geometries of the BenzeneEthene dimer in the S66x 8 benchmark. Each ML model is compared to the level of theory used for parameterization: $\omega \mathrm{B} 97 \mathrm{X} / 6-31 \mathrm{G}^{*}$ for ANI-1X and SAPT0/jun-cc-pVDZ for AP-Net.

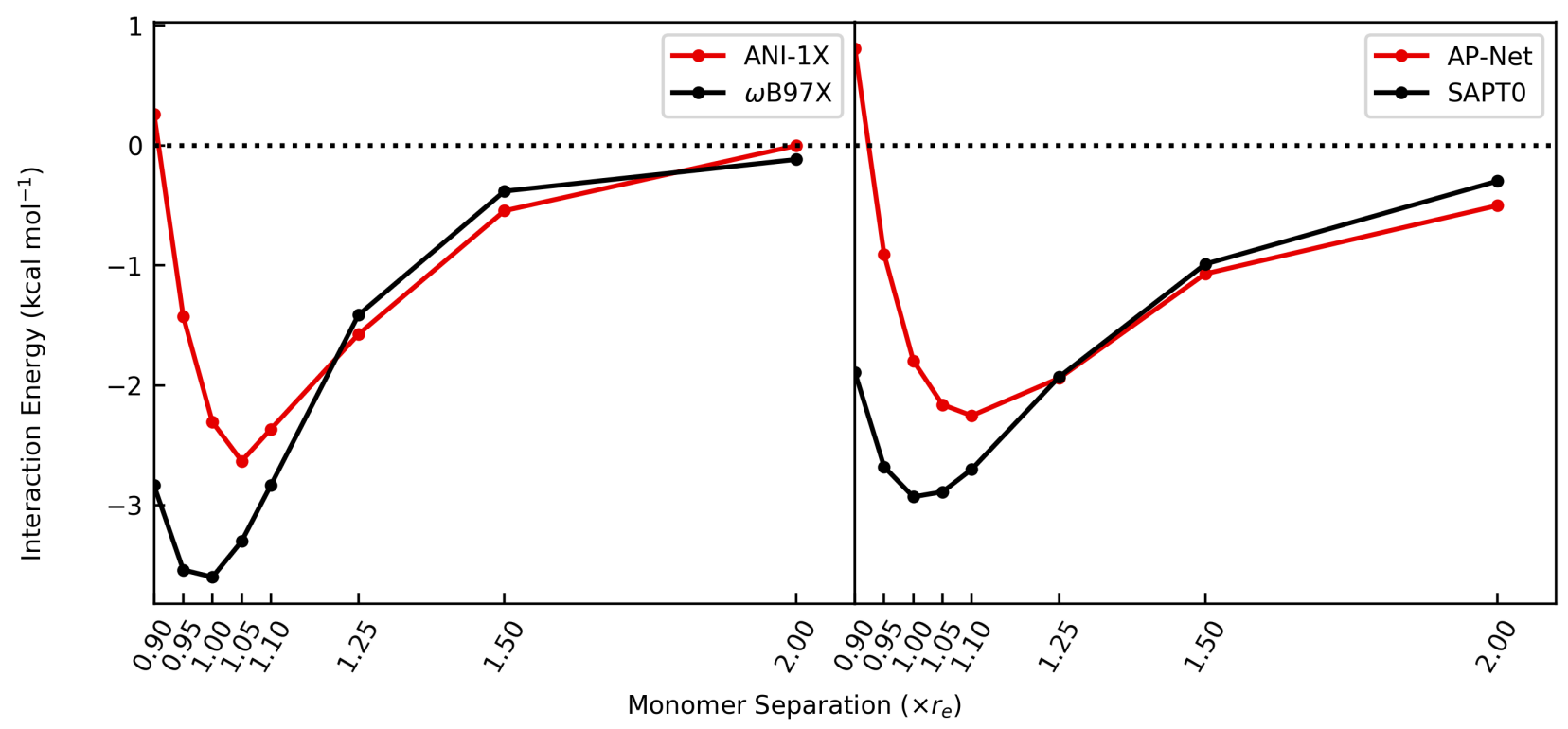

FIG. S32. ANI-1X and AP-Net are evaluated on the 8 geometries of the UracilEthene dimer in the S66x8 benchmark. Each ML model is compared to the level of theory used for parameterization: $\omega \mathrm{B} 97 \mathrm{X} / 6-31 \mathrm{G} *$ for ANI-1X and SAPT0/jun-cc-pVDZ for AP-Net. 


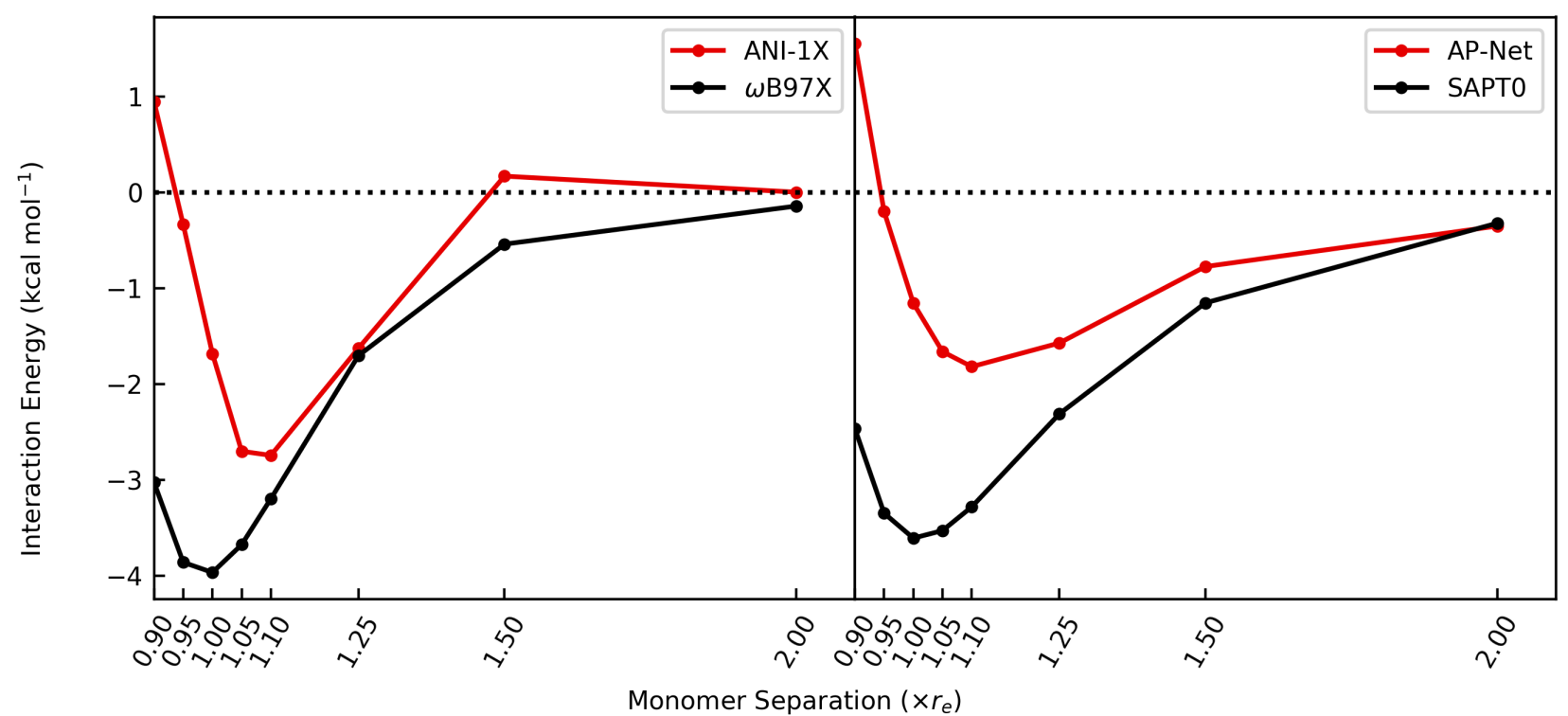

FIG. S33. ANI-1X and AP-Net are evaluated on the 8 geometries of the UracilEthyne dimer in the S66x8 benchmark. Each ML model is compared to the level of theory used for parameterization: $\omega \mathrm{B} 97 \mathrm{X} / 6-31 \mathrm{G}^{*}$ for ANI-1X and SAPT0/jun-cc-pVDZ for AP-Net.

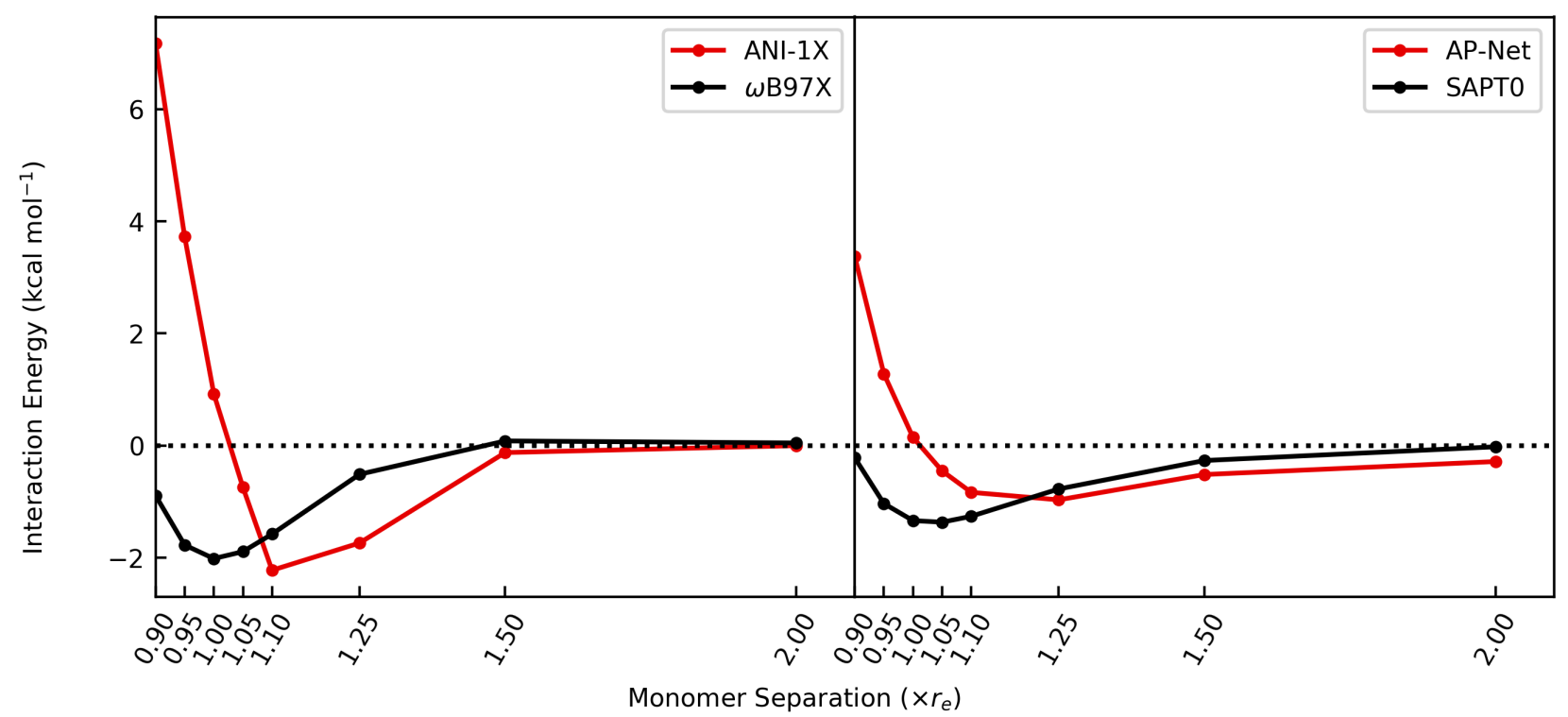

FIG. S34. ANI-1X and AP-Net are evaluated on the 8 geometries of the PyridineEthene dimer in the S66x 8 benchmark. Each ML model is compared to the level of theory used for parameterization: $\omega \mathrm{B} 97 \mathrm{X} / 6-31 \mathrm{G}^{*}$ for ANI-1X and SAPT0/jun-cc-pVDZ for AP-Net. 


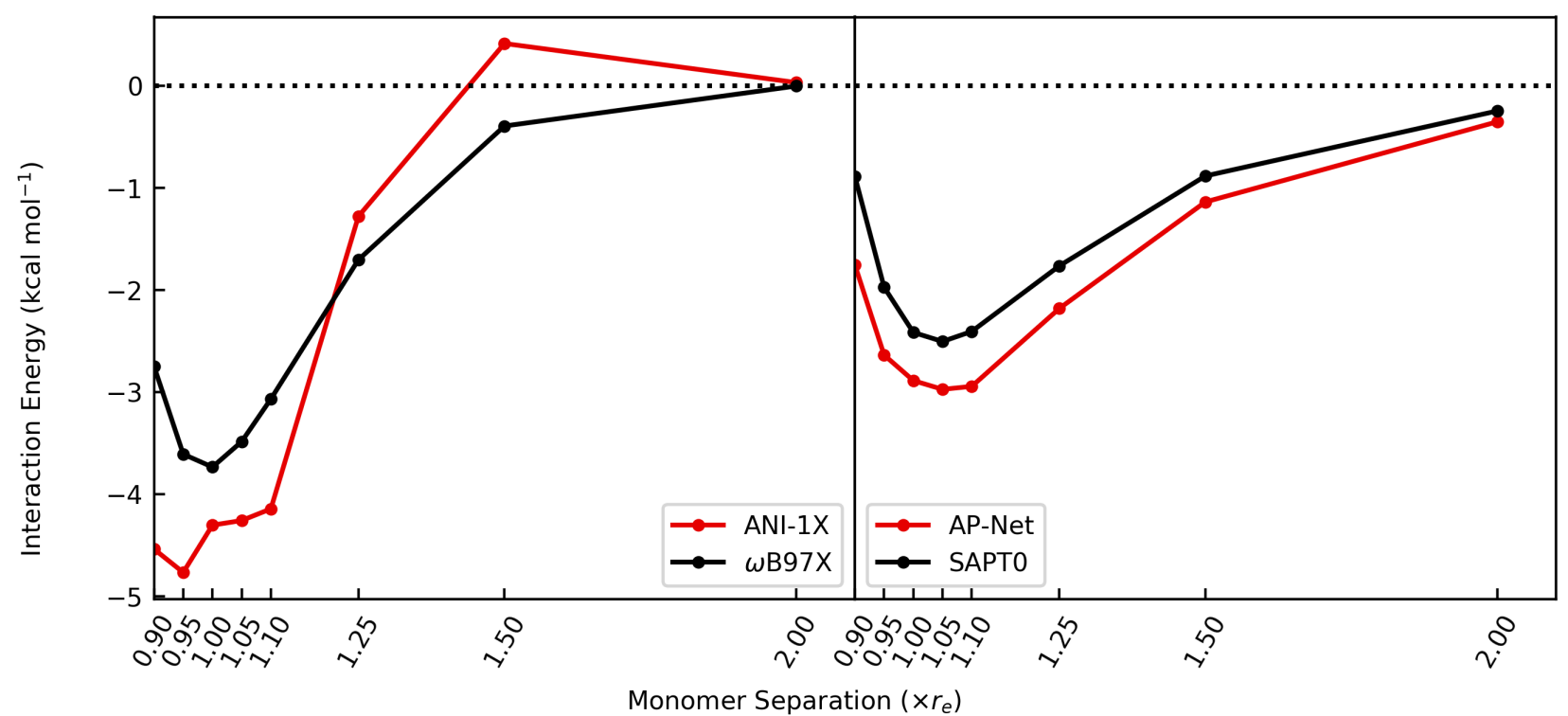

FIG. S35. ANI-1X and AP-Net are evaluated on the 8 geometries of the PentanePentane dimer in the S66x 8 benchmark. Each ML model is compared to the level of theory used for parameterization: $\omega \mathrm{B} 97 \mathrm{X} / 6-31 \mathrm{G}^{*}$ for ANI-1X and SAPT0/jun-cc-pVDZ for AP-Net.

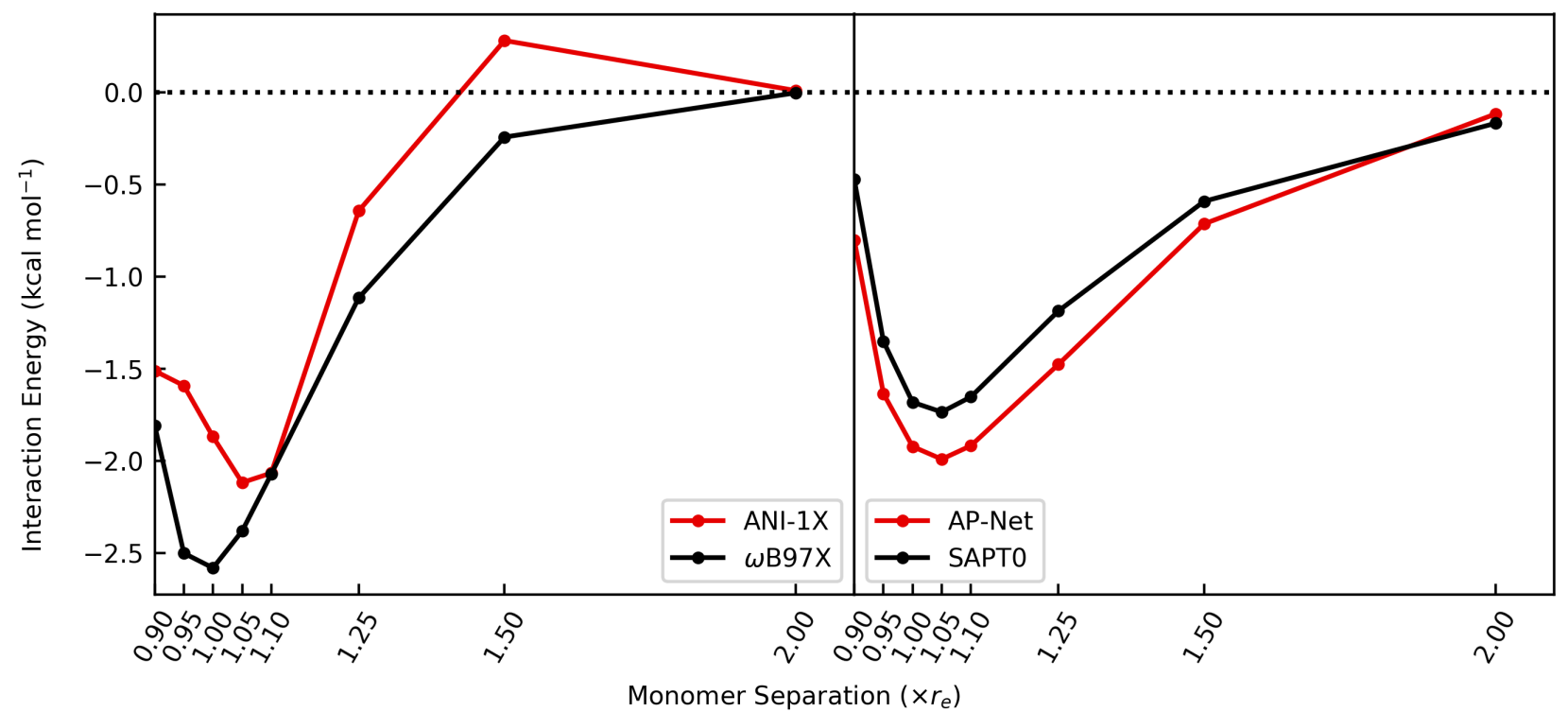

FIG. S36. ANI-1X and AP-Net are evaluated on the 8 geometries of the NeopentanePentane dimer in the S66x8 benchmark. Each ML model is compared to the level of theory used for parameterization: $\omega$ B97X/631G* for ANI-1X and SAPT0/jun-cc-pVDZ for AP-Net. 


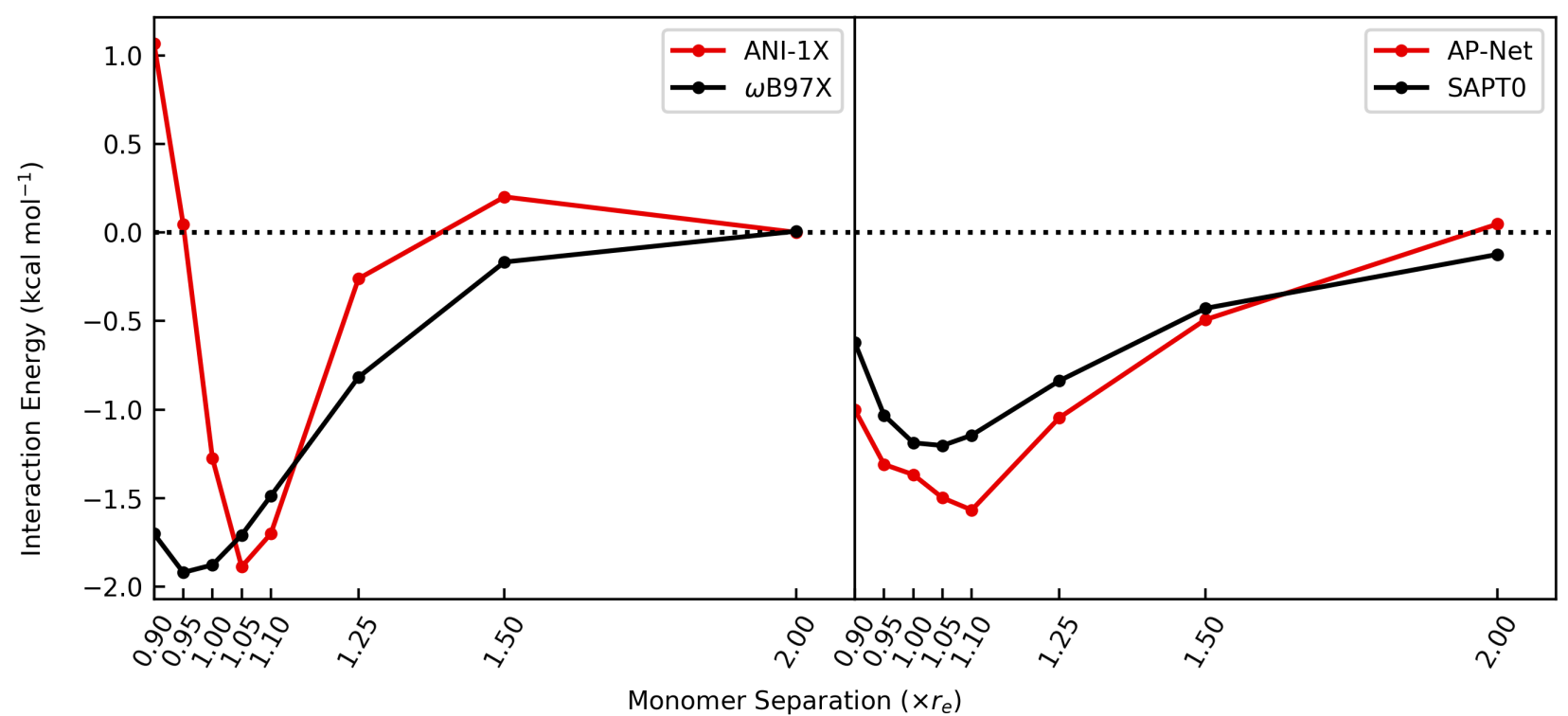

FIG. S37. ANI-1X and AP-Net are evaluated on the 8 geometries of the NeopentaneNeopentane dimer in the S66x8 benchmark. Each ML model is compared to the level of theory used for parameterization: $\omega \mathrm{B} 97 \mathrm{X} / 6-31 \mathrm{G}^{*}$ for ANI-1X and SAPT0/jun-cc-pVDZ for AP-Net.

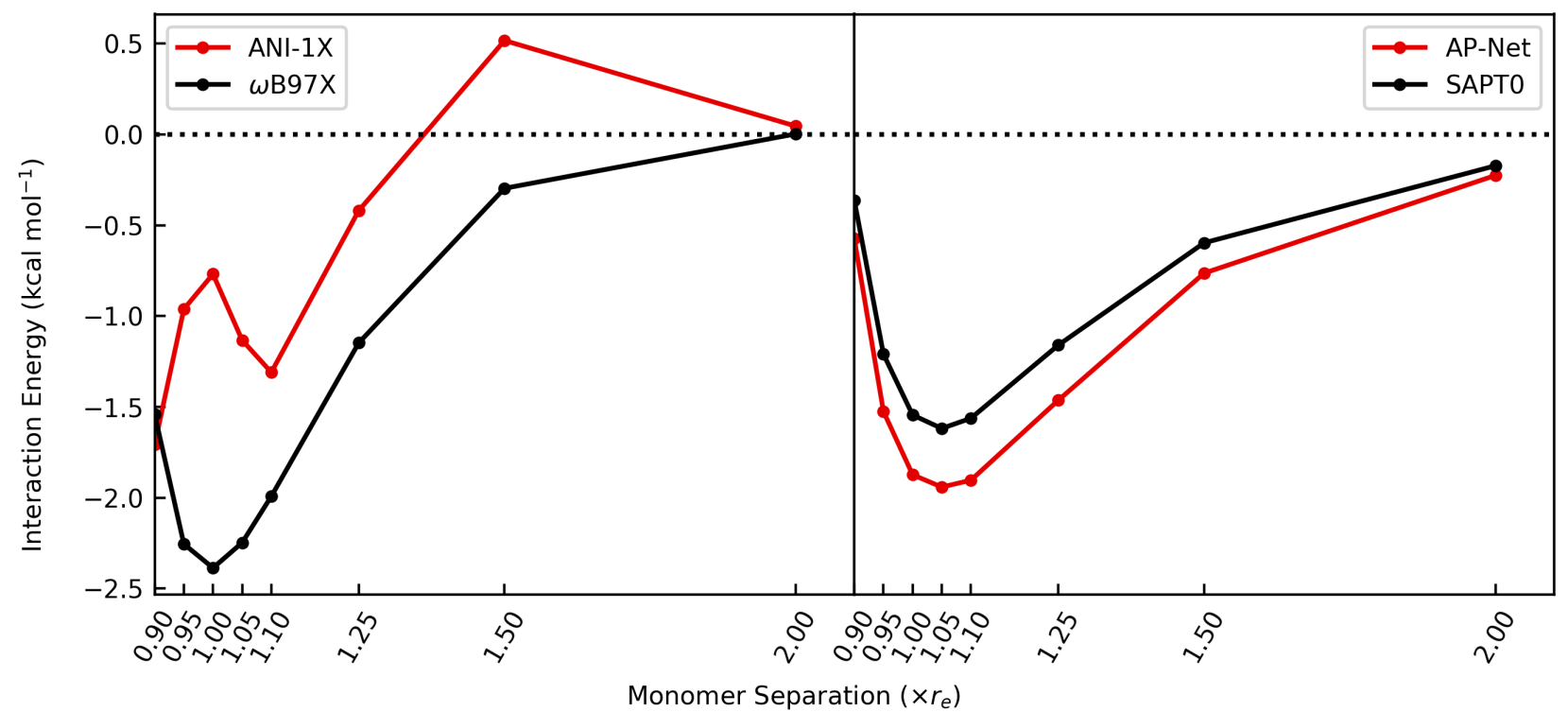

FIG. S38. ANI-1X and AP-Net are evaluated on the 8 geometries of the CyclopentaneNeopentane dimer in the S66x8 benchmark. Each ML model is compared to the level of theory used for parameterization: $\omega \mathrm{B} 97 \mathrm{X} / 6-31 \mathrm{G}^{*}$ for ANI-1X and SAPT0/jun-cc-pVDZ for AP-Net. 


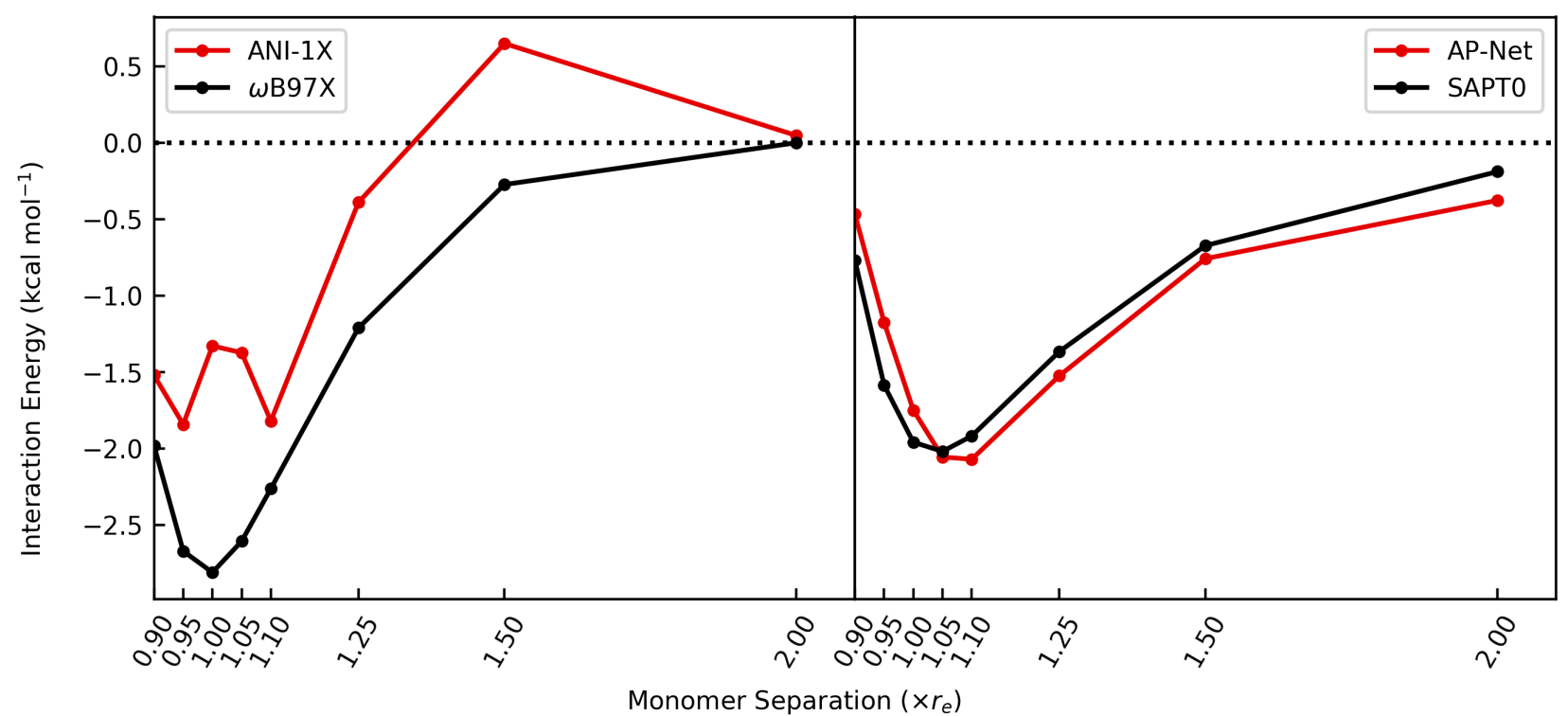

FIG. S39. ANI-1X and AP-Net are evaluated on the 8 geometries of the CyclopentaneCyclopentane dimer in the S66x8 benchmark. Each ML model is compared to the level of theory used for parameterization: $\omega \mathrm{B} 97 \mathrm{X} / 6-31 \mathrm{G}^{*}$ for ANI-1X and SAPT0/jun-cc-pVDZ for AP-Net.

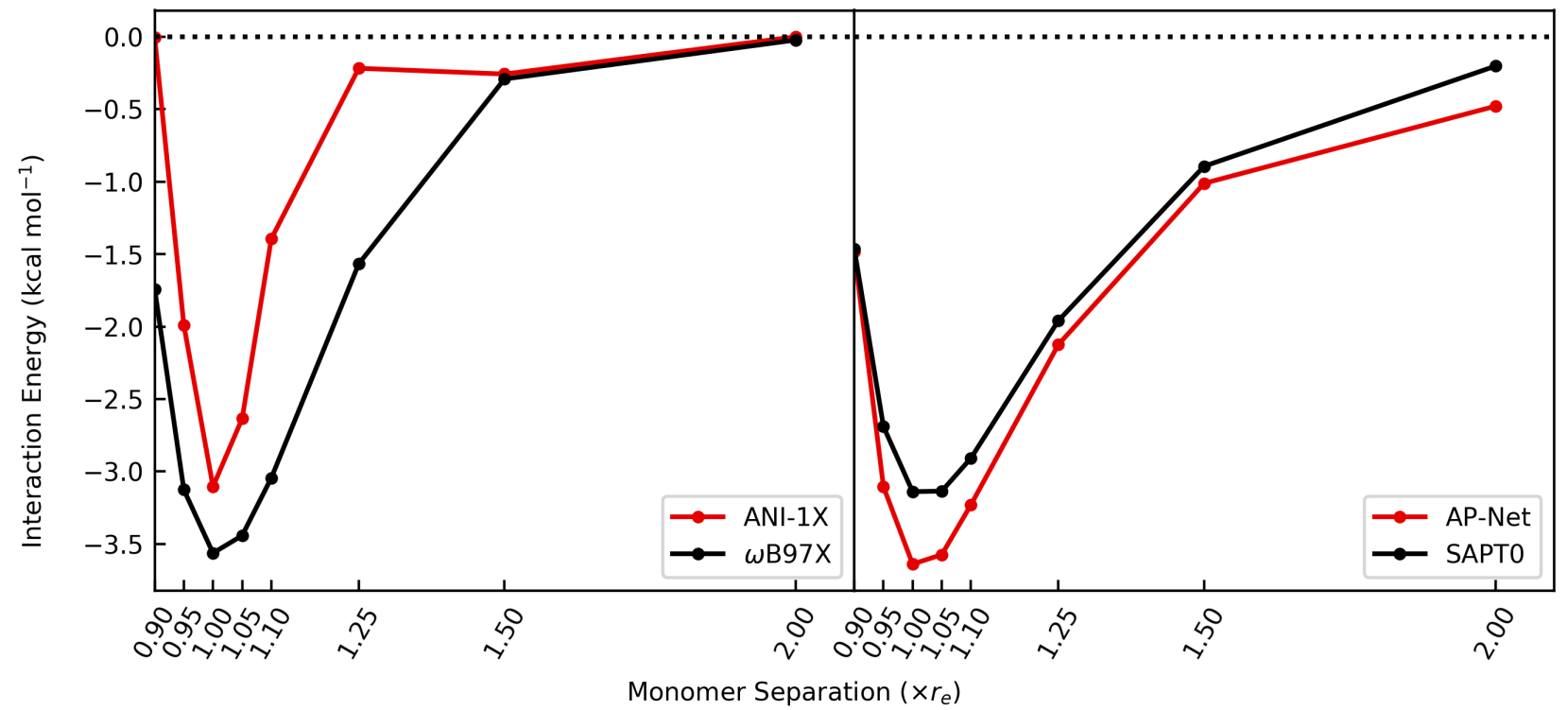

FIG. S40. ANI-1X and AP-Net are evaluated on the 8 geometries of the BenzeneCyclopentane dimer in the S66x8 benchmark. Each ML model is compared to the level of theory used for parameterization: $\omega$ B97X/631G* for ANI-1X and SAPT0/jun-cc-pVDZ for AP-Net. 


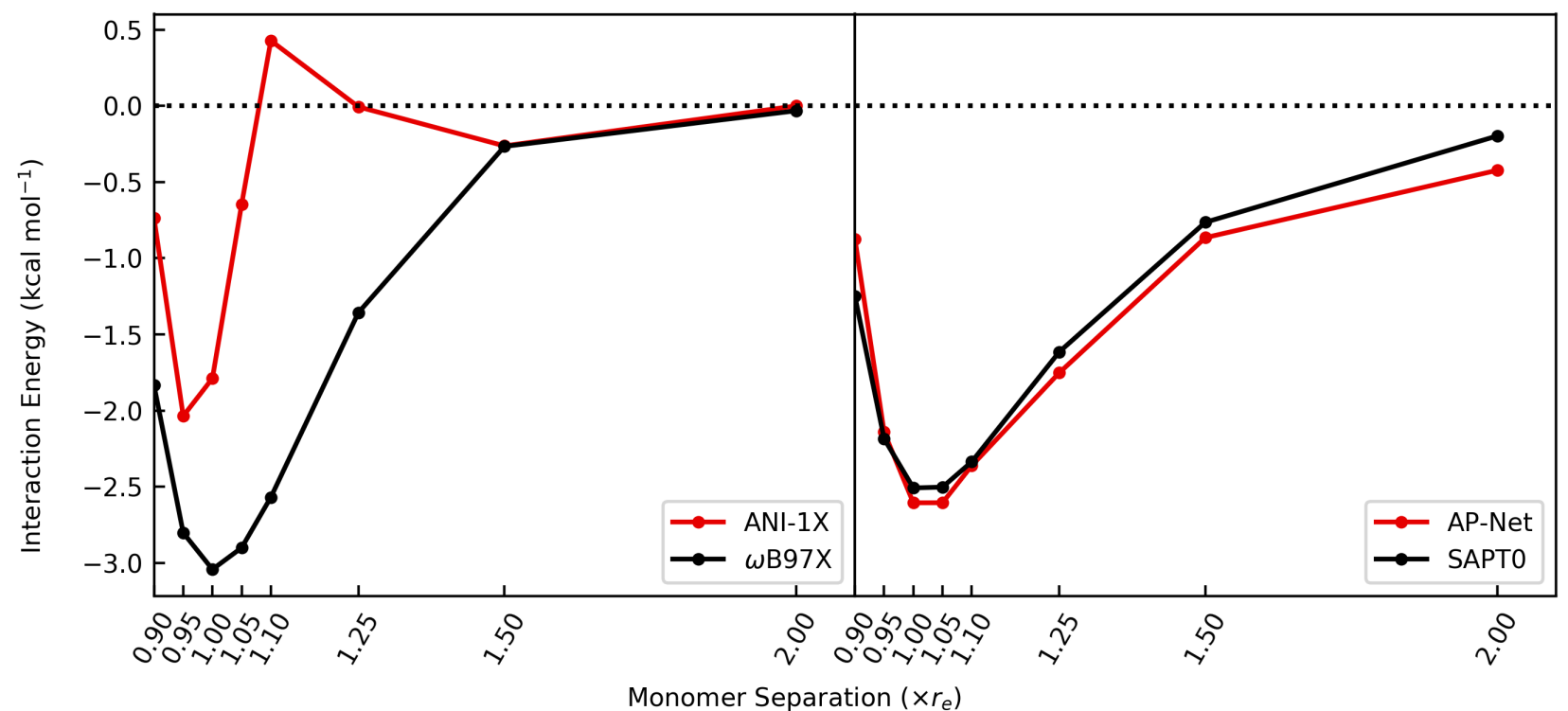

FIG. S41. ANI-1X and AP-Net are evaluated on the 8 geometries of the BenzeneNeopentane dimer in the S66x8 benchmark. Each ML model is compared to the level of theory used for parameterization: $\omega$ B97X/631G* for ANI-1X and SAPT0/jun-cc-pVDZ for AP-Net.

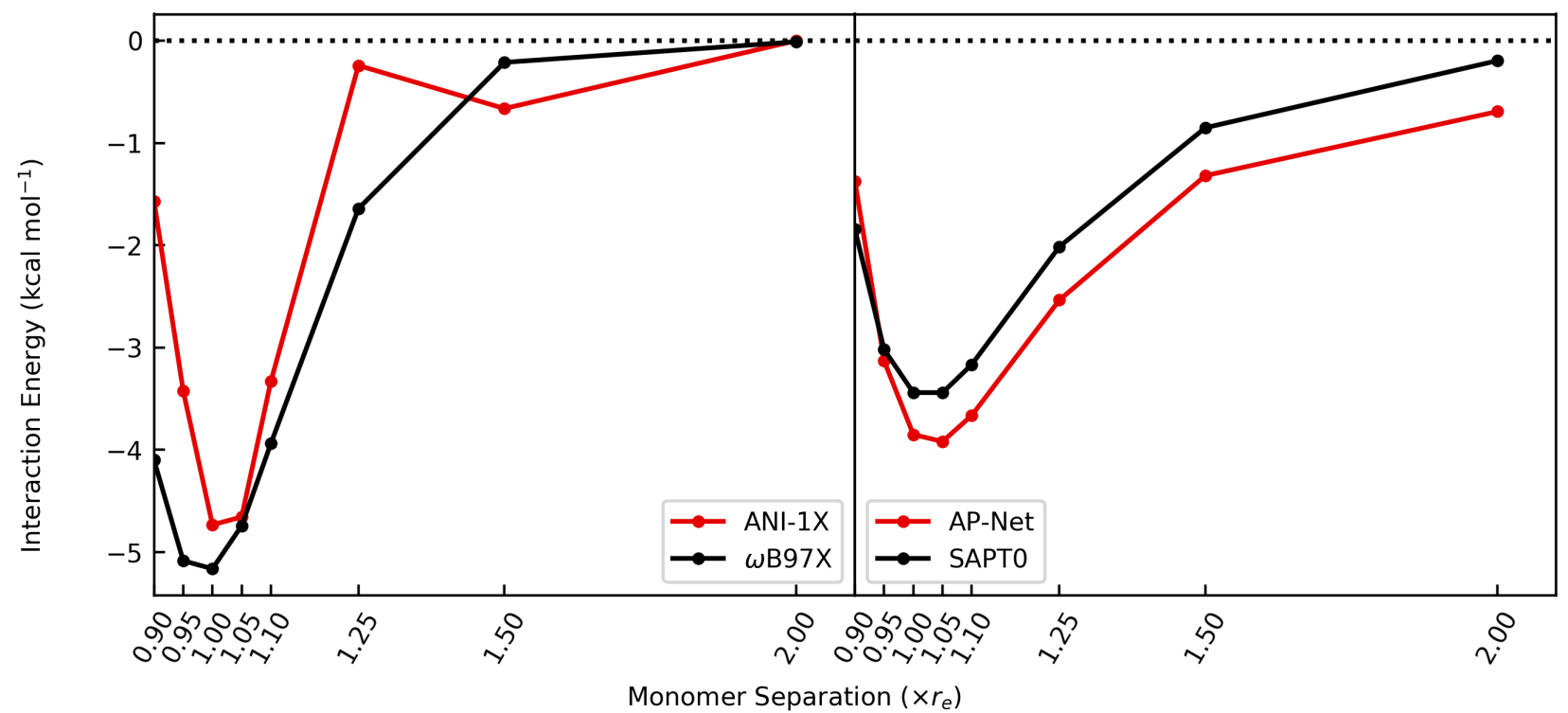

FIG. S42. ANI-1X and AP-Net are evaluated on the 8 geometries of the UracilPentane dimer in the S66x8 benchmark. Each ML model is compared to the level of theory used for parameterization: $\omega \mathrm{B} 97 \mathrm{X} / 6-31 \mathrm{G}^{*}$ for ANI-1X and SAPT0/jun-cc-pVDZ for AP-Net. 


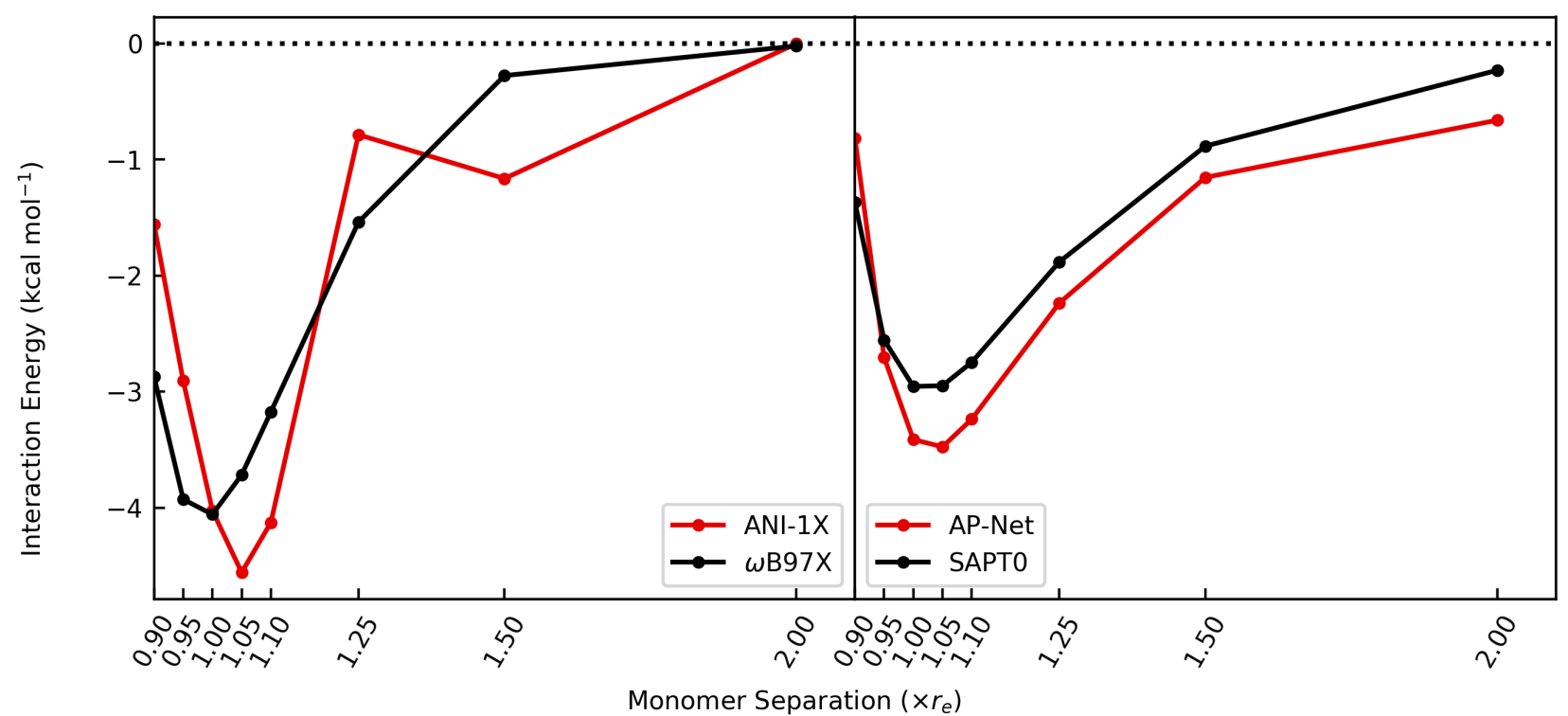

FIG. S43. ANI-1X and AP-Net are evaluated on the 8 geometries of the UracilCyclopentane dimer in the S66x8 benchmark. Each ML model is compared to the level of theory used for parameterization: $\omega$ B97X/631G* for ANI-1X and SAPT0/jun-cc-pVDZ for AP-Net.

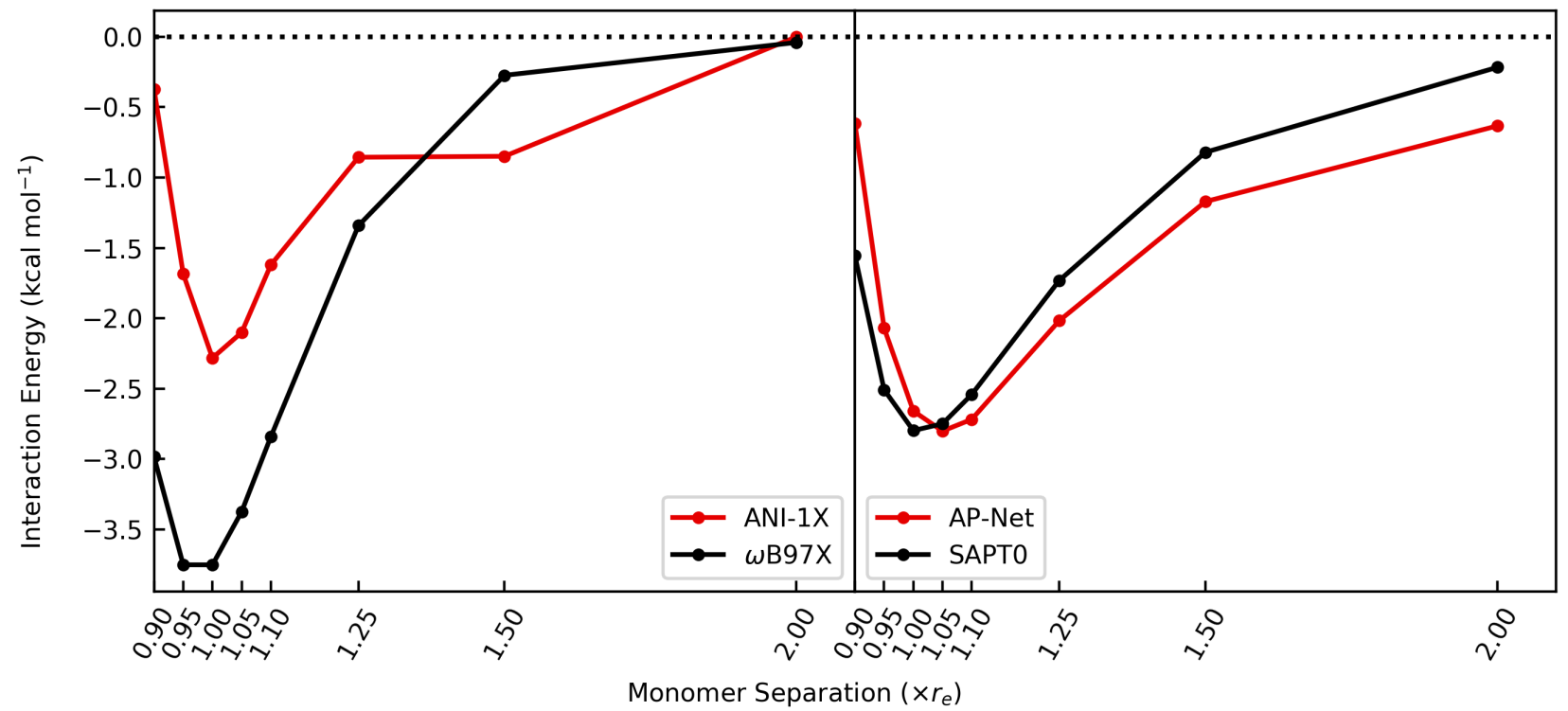

FIG. S44. ANI-1X and AP-Net are evaluated on the 8 geometries of the UracilNeopentane dimer in the S66x8 benchmark. Each ML model is compared to the level of theory used for parameterization: $\omega$ B97X/631G* for ANI-1X and SAPT0/jun-cc-pVDZ for AP-Net. 


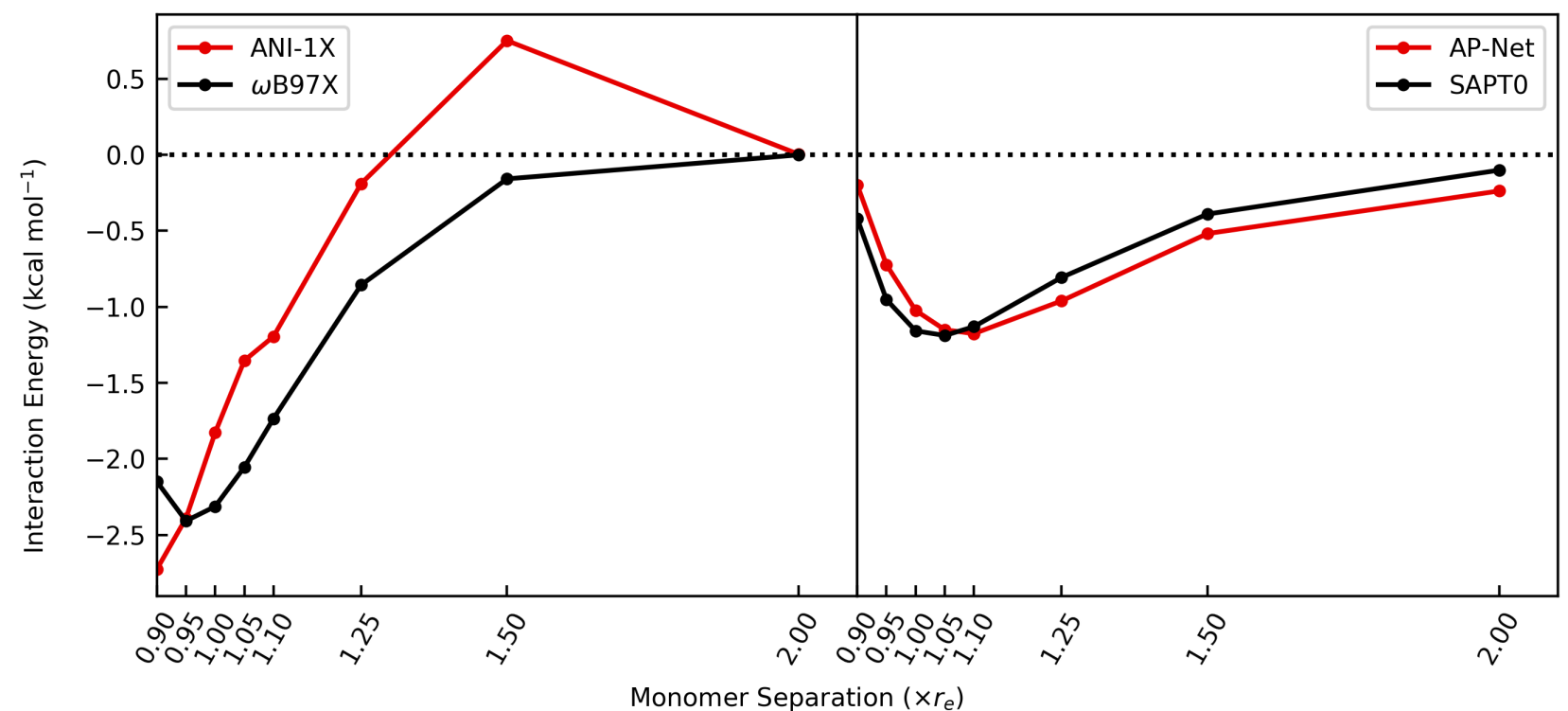

FIG. S45. ANI-1X and AP-Net are evaluated on the 8 geometries of the EthenePentane dimer in the S66x8 benchmark. Each ML model is compared to the level of theory used for parameterization: $\omega \mathrm{B} 97 \mathrm{X} / 6-31 \mathrm{G}^{*}$ for ANI-1X and SAPT0/jun-cc-pVDZ for AP-Net.

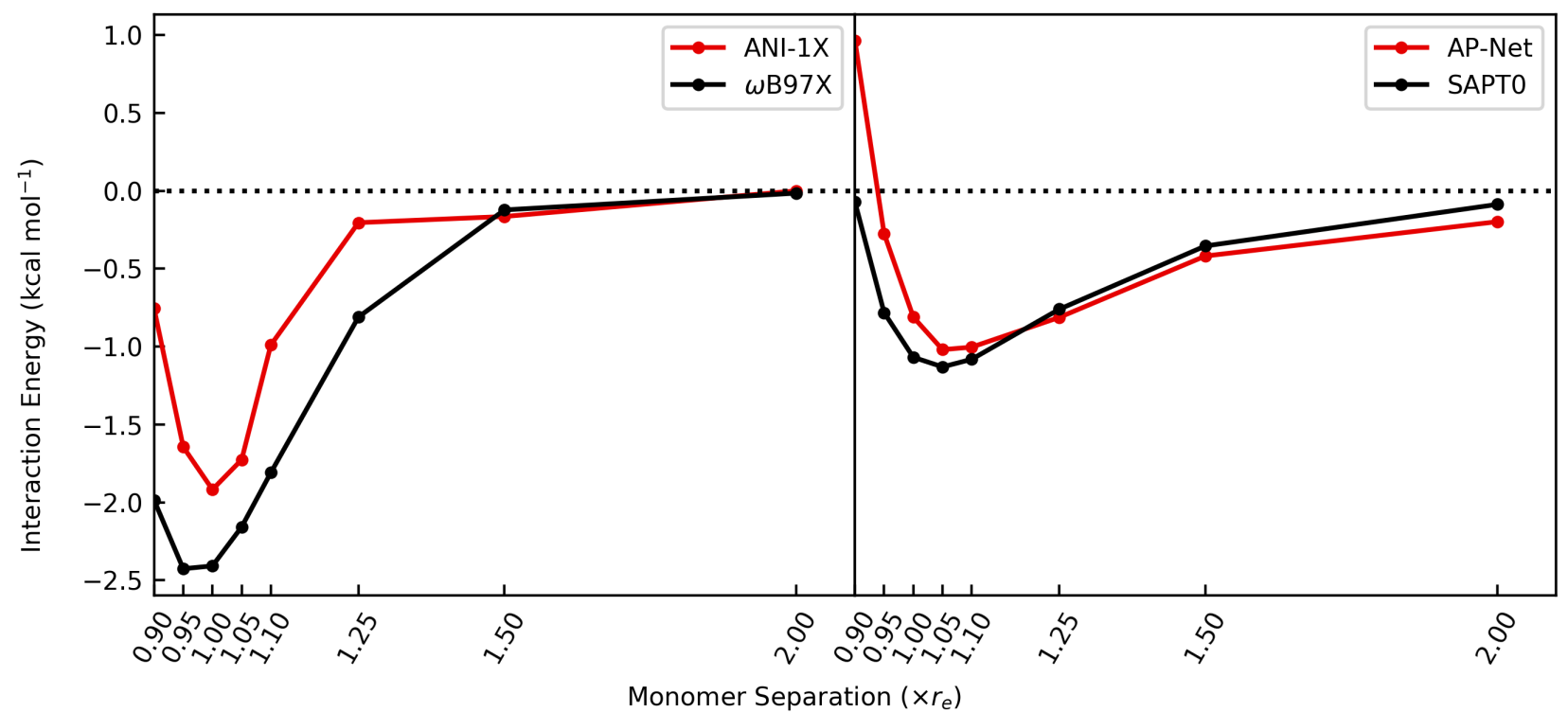

FIG. S46. ANI-1X and AP-Net are evaluated on the 8 geometries of the EthynePentane dimer in the S66x 8 benchmark. Each ML model is compared to the level of theory used for parameterization: $\omega \mathrm{B} 97 \mathrm{X} / 6-31 \mathrm{G}^{*}$ for ANI-1X and SAPT0/jun-cc-pVDZ for AP-Net. 


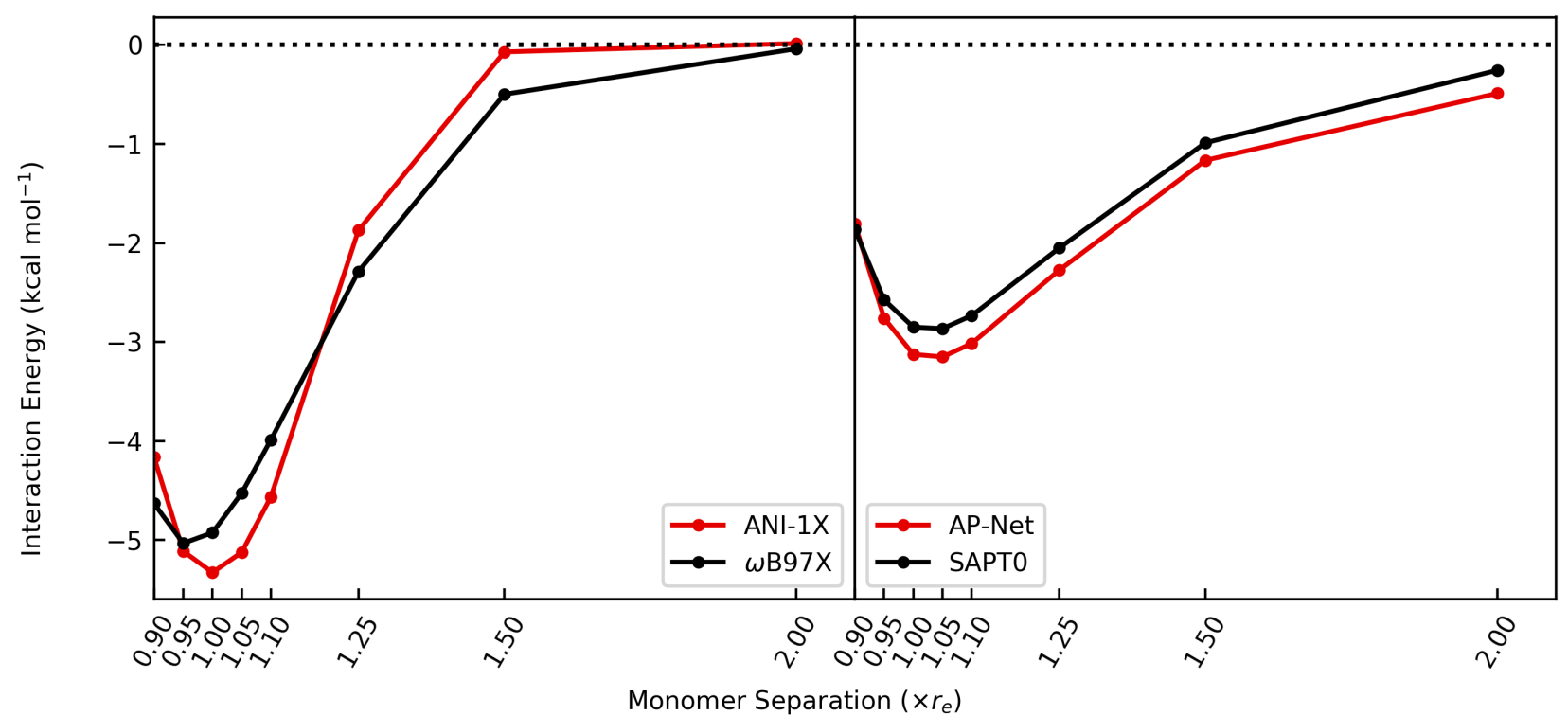

FIG. S47. ANI-1X and AP-Net are evaluated on the 8 geometries of the PeptidePentane dimer in the S66x8 benchmark. Each ML model is compared to the level of theory used for parameterization: $\omega \mathrm{B} 97 \mathrm{X} / 6-31 \mathrm{G}^{*}$ for ANI-1X and SAPT0/jun-cc-pVDZ for AP-Net.

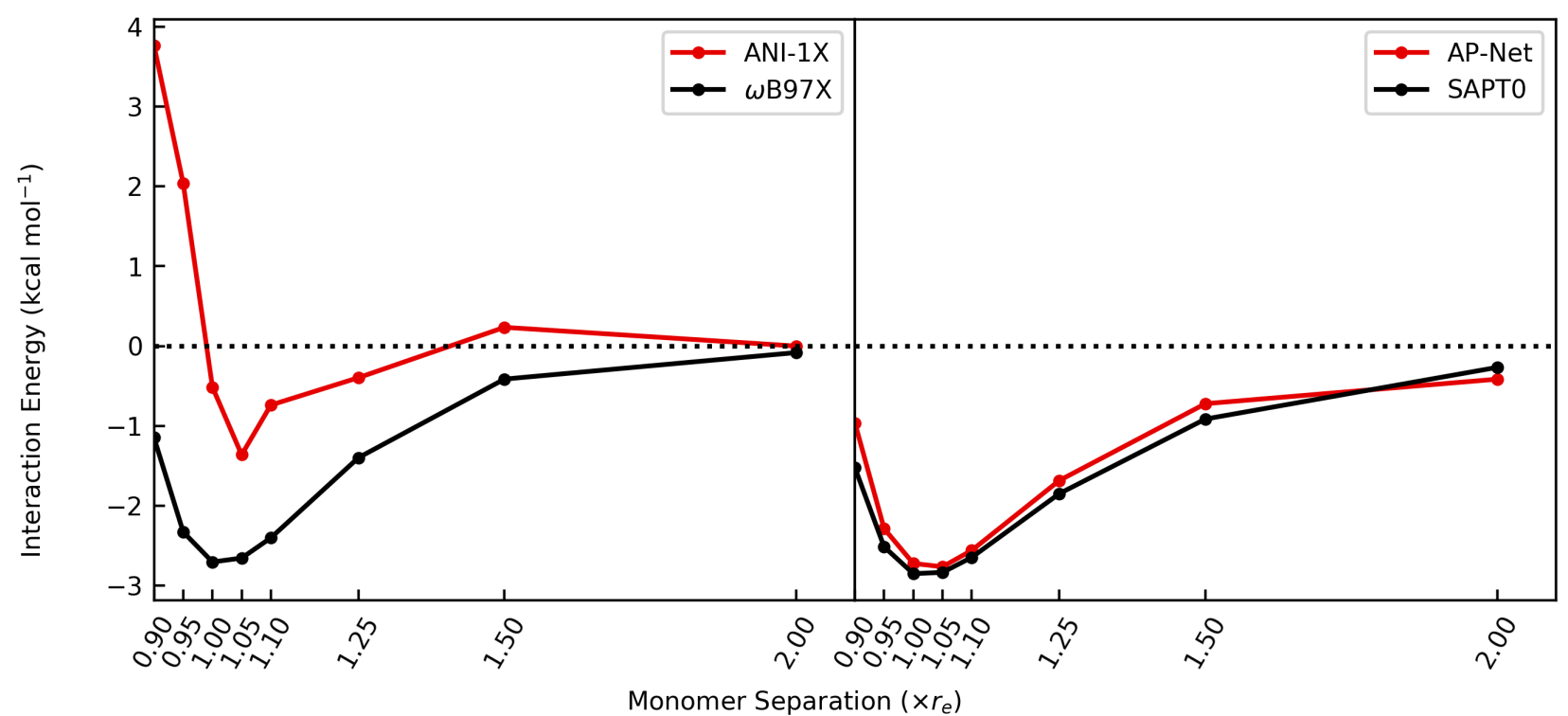

FIG. S48. ANI-1X and AP-Net are evaluated on the 8 geometries of the BenzeneBenzeneTS dimer in the S66x8 benchmark. Each ML model is compared to the level of theory used for parameterization: $\omega$ B97X/631G* for ANI-1X and SAPT0/jun-cc-pVDZ for AP-Net. 


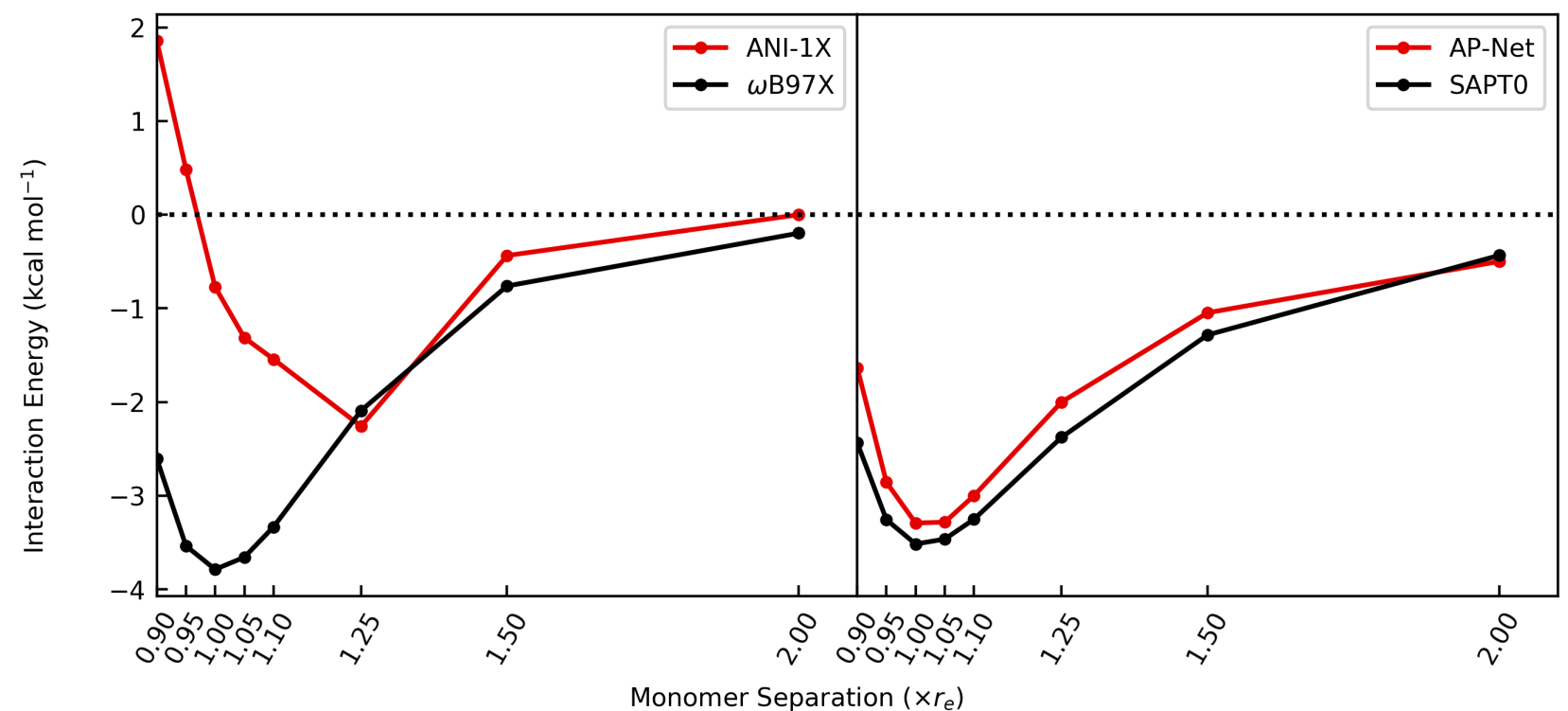

FIG. S49. ANI-1X and AP-Net are evaluated on the 8 geometries of the PyridinePyridineTS dimer in the S66x8 benchmark. Each ML model is compared to the level of theory used for parameterization: $\omega$ B97X/631G* for ANI-1X and SAPT0/jun-cc-pVDZ for AP-Net.

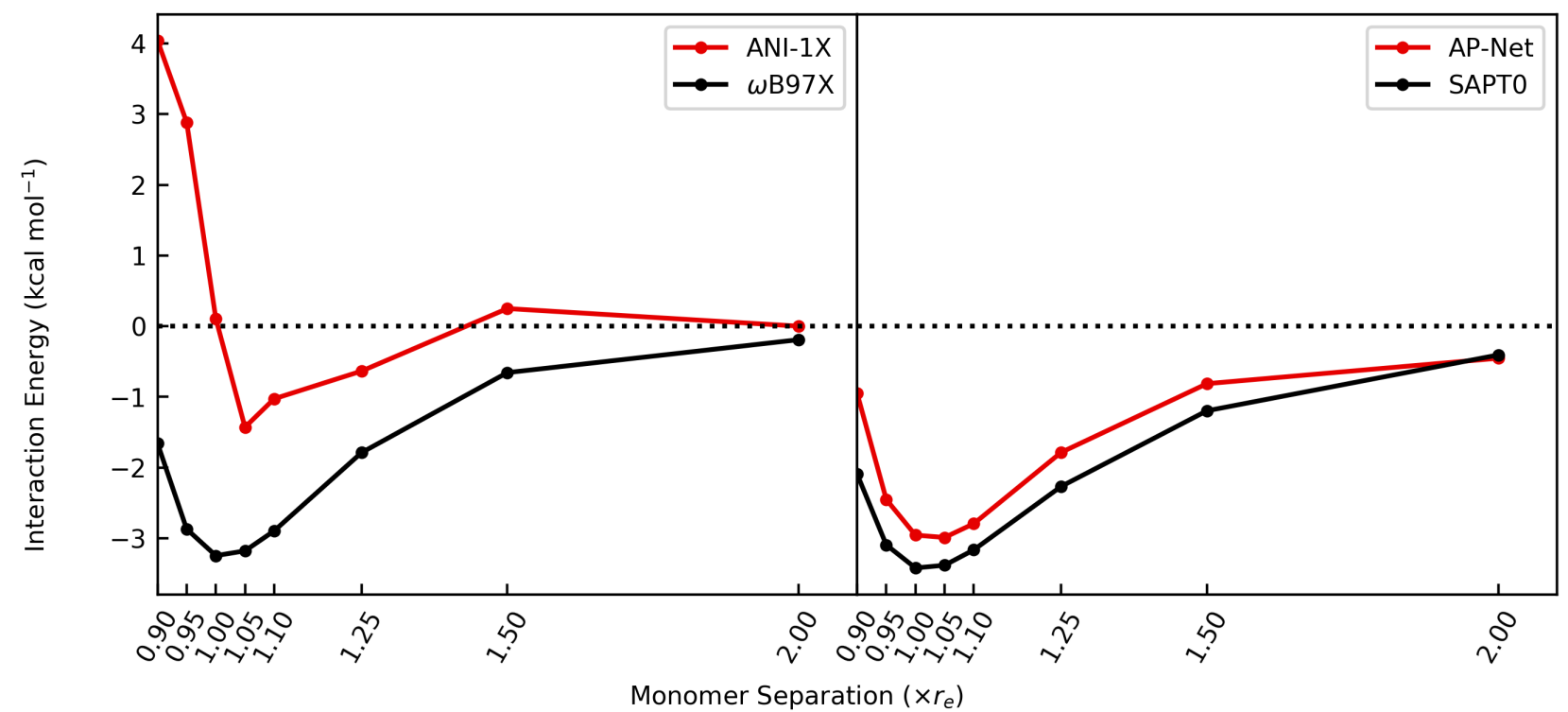

FIG. S50. ANI-1X and AP-Net are evaluated on the 8 geometries of the BenzenePyridineTS dimer in the S66x8 benchmark. Each ML model is compared to the level of theory used for parameterization: $\omega$ B97X/631G* for ANI-1X and SAPT0/jun-cc-pVDZ for AP-Net. 


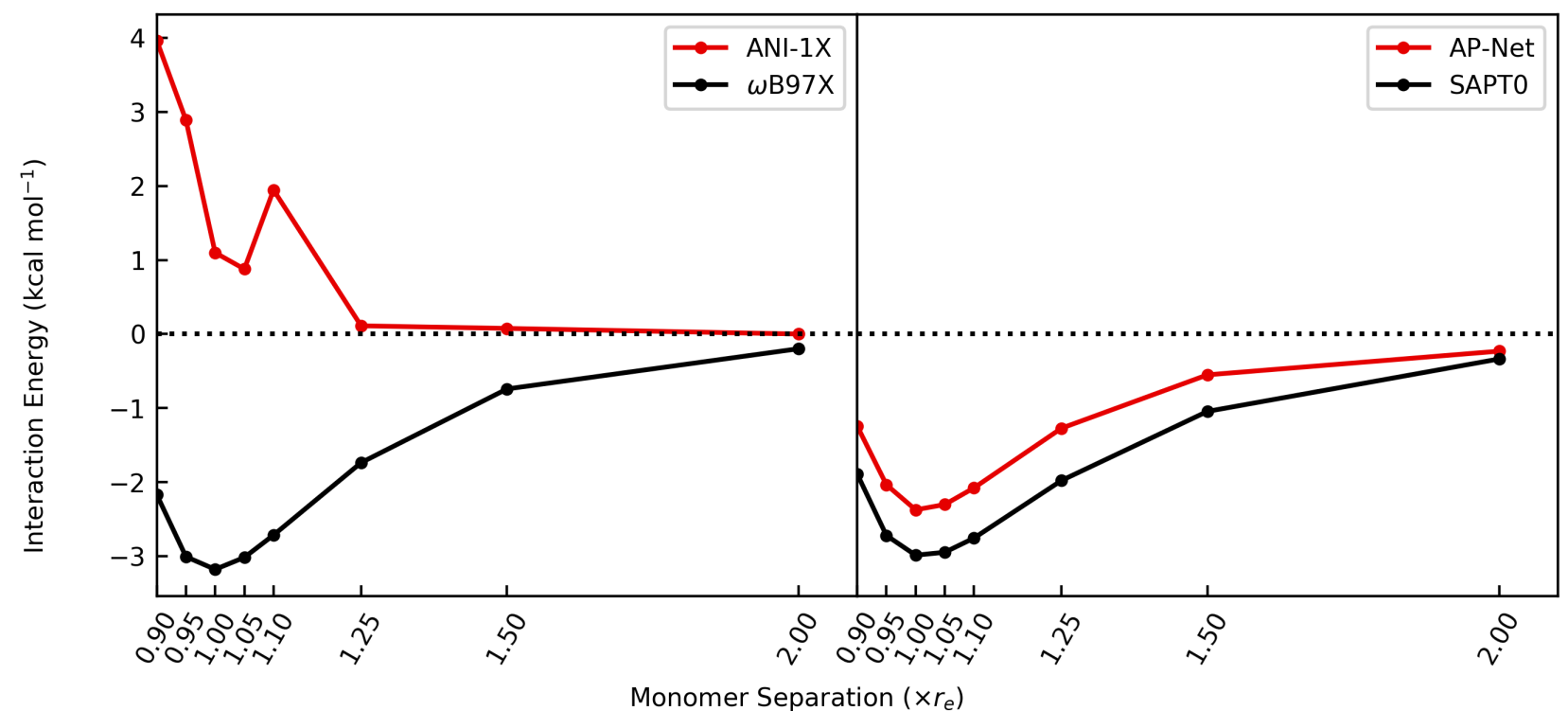

FIG. S51. ANI-1X and AP-Net are evaluated on the 8 geometries of the BenzeneEthyneCHpi dimer in the S66x8 benchmark. Each ML model is compared to the level of theory used for parameterization: $\omega$ B97X/631G* for ANI-1X and SAPT0/jun-cc-pVDZ for AP-Net.

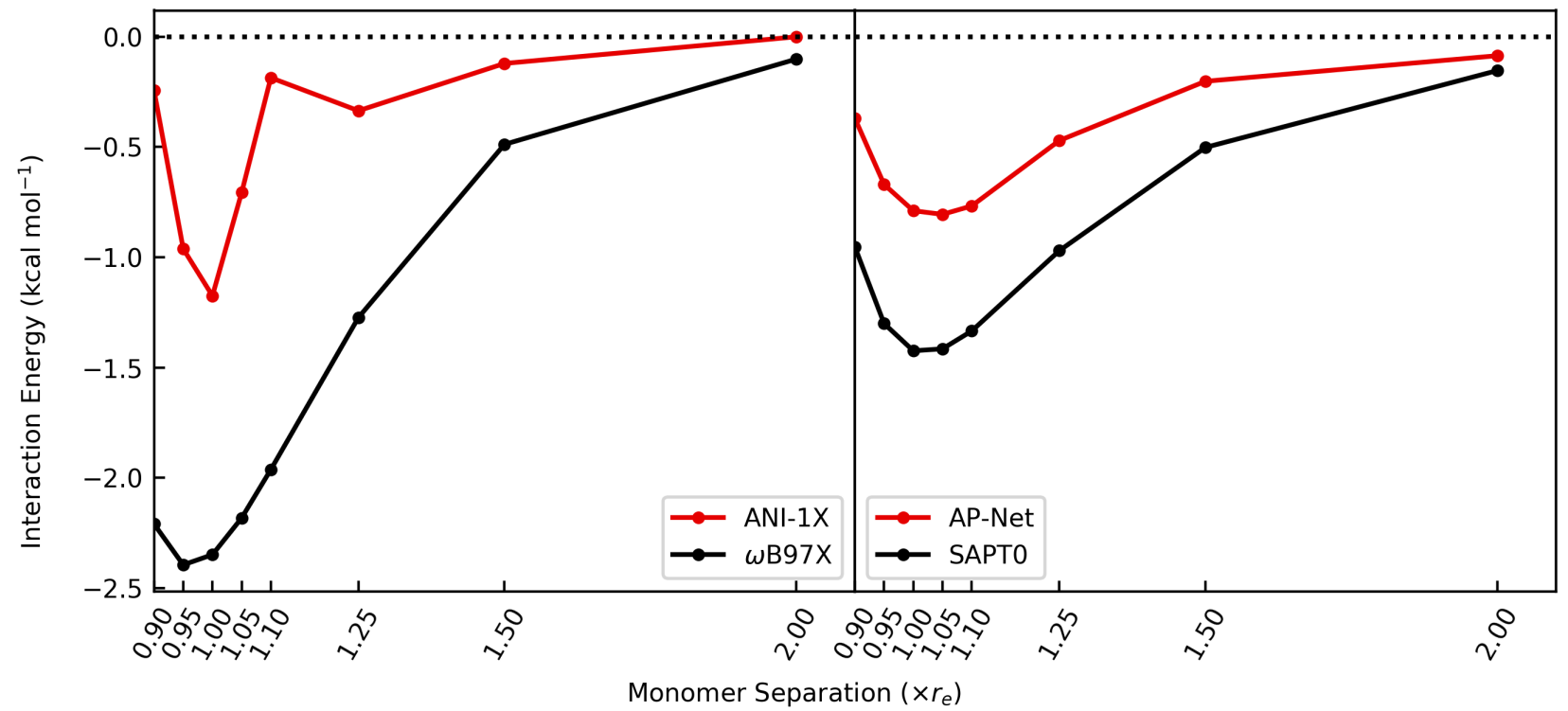

FIG. S52. ANI-1X and AP-Net are evaluated on the 8 geometries of the EthyneEthyneTS dimer in the S66x 8 benchmark. Each ML model is compared to the level of theory used for parameterization: $\omega \mathrm{B} 97 \mathrm{X} / 6-31 \mathrm{G}^{*}$ for ANI-1X and SAPT0/jun-cc-pVDZ for AP-Net. 


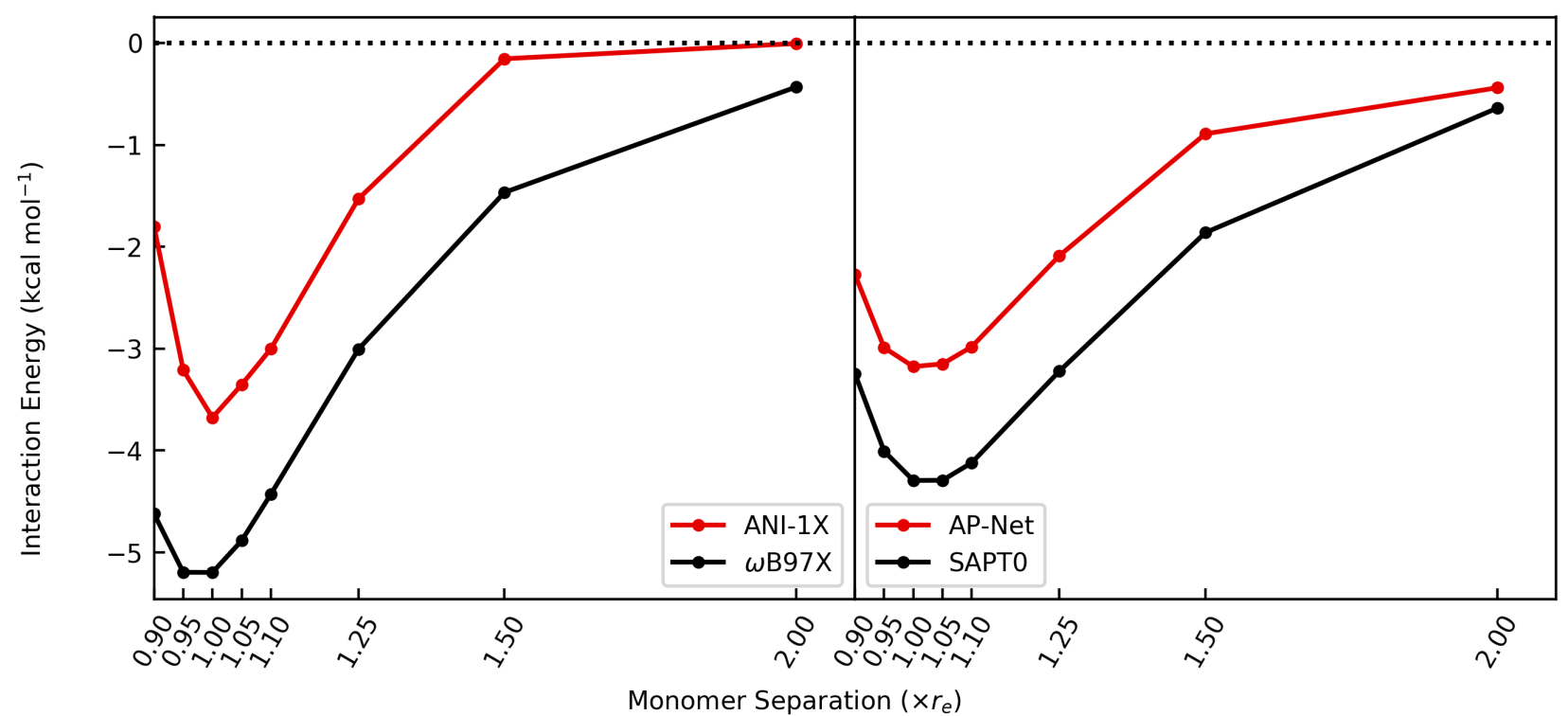

FIG. S53. ANI-1X and AP-Net are evaluated on the 8 geometries of the BenzeneAcOHOHpi dimer in the S66x8 benchmark. Each ML model is compared to the level of theory used for parameterization: $\omega$ B97X/631G* for ANI-1X and SAPT0/jun-cc-pVDZ for AP-Net.

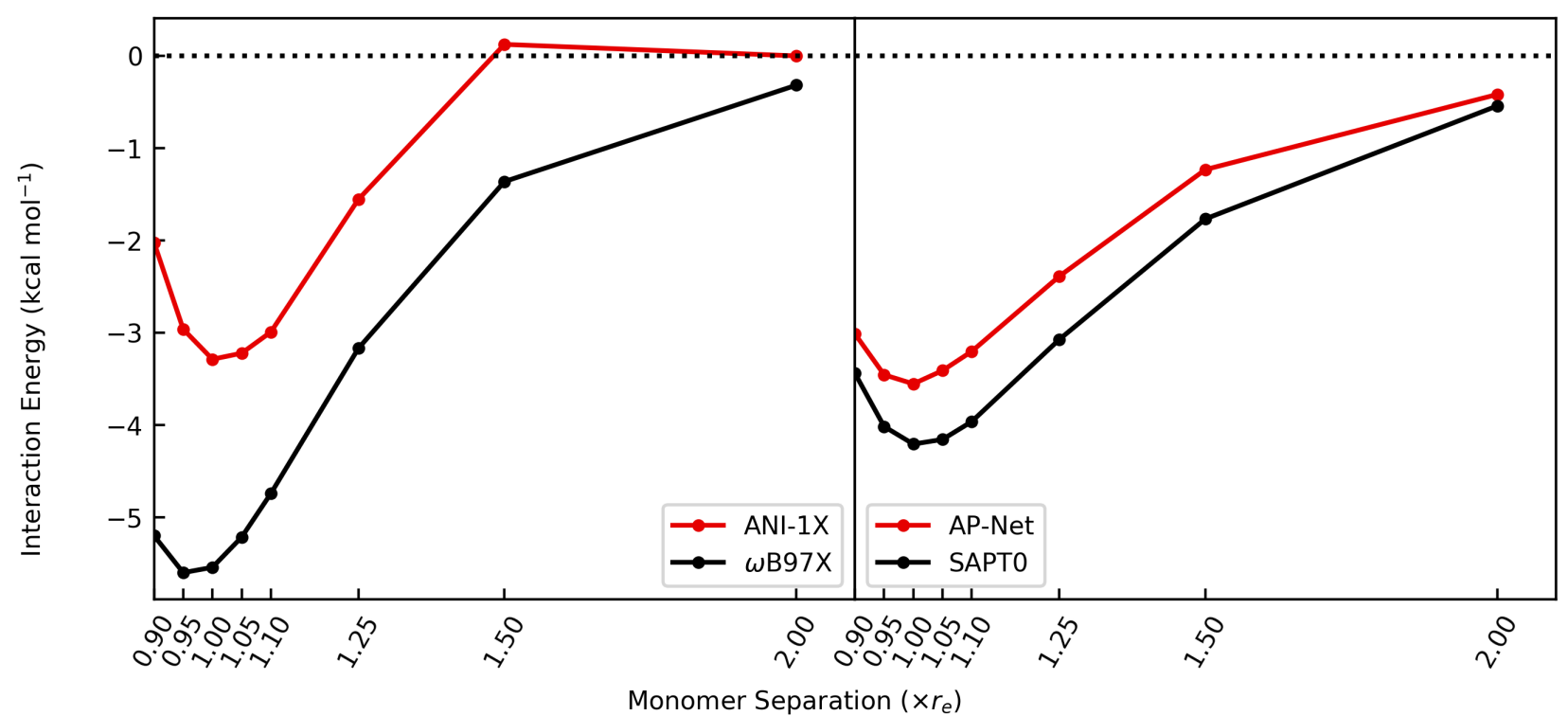

FIG. S54. ANI-1X and AP-Net are evaluated on the 8 geometries of the BenzeneAcNH2NHpi dimer in the S66x8 benchmark. Each ML model is compared to the level of theory used for parameterization: $\omega$ B97X/631G* for ANI-1X and SAPT0/jun-cc-pVDZ for AP-Net. 




FIG. S55. ANI-1X and AP-Net are evaluated on the 8 geometries of the BenzeneWaterOHpi dimer in the S66x8 benchmark. Each ML model is compared to the level of theory used for parameterization: $\omega$ B97X/631G* for ANI-1X and SAPT0/jun-cc-pVDZ for AP-Net.

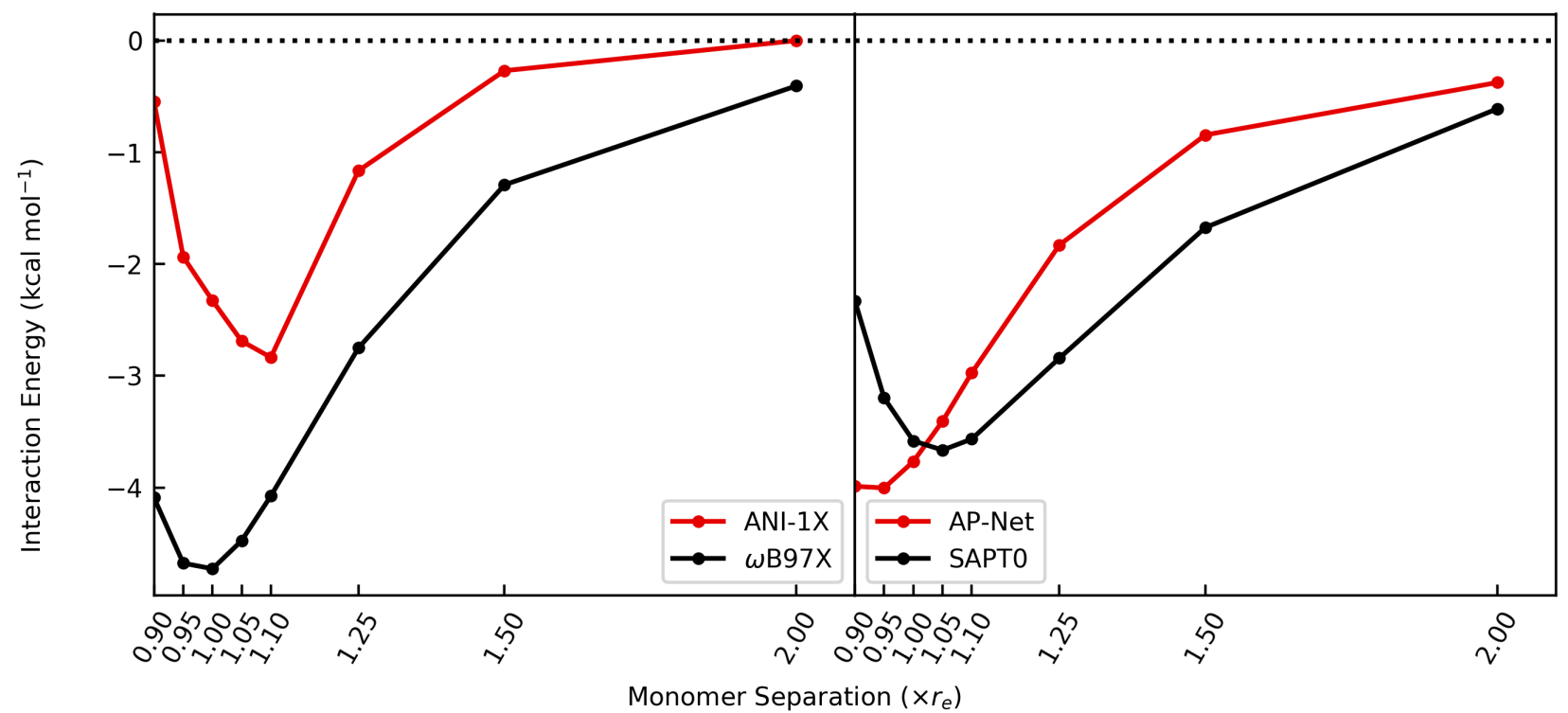

FIG. S56. ANI-1X and AP-Net are evaluated on the 8 geometries of the BenzeneMeOHOHpi dimer in the S66x8 benchmark. Each ML model is compared to the level of theory used for parameterization: $\omega$ B97X/631G* for ANI-1X and SAPT0/jun-cc-pVDZ for AP-Net. 


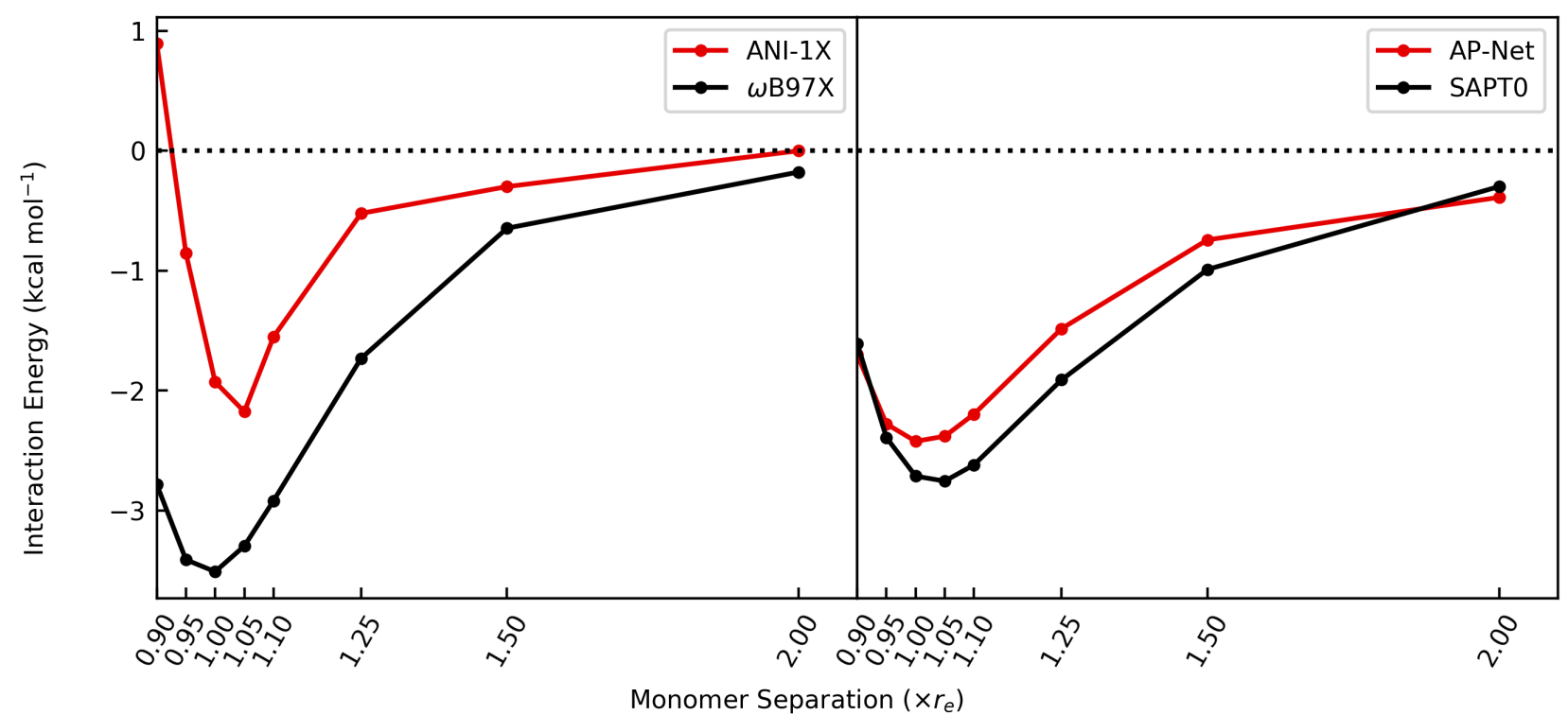

FIG. S57. ANI-1X and AP-Net are evaluated on the 8 geometries of the BenzeneMeNH2NHpi dimer in the S66x8 benchmark. Each ML model is compared to the level of theory used for parameterization: $\omega$ B97X/631G* for ANI-1X and SAPT0/jun-cc-pVDZ for AP-Net.

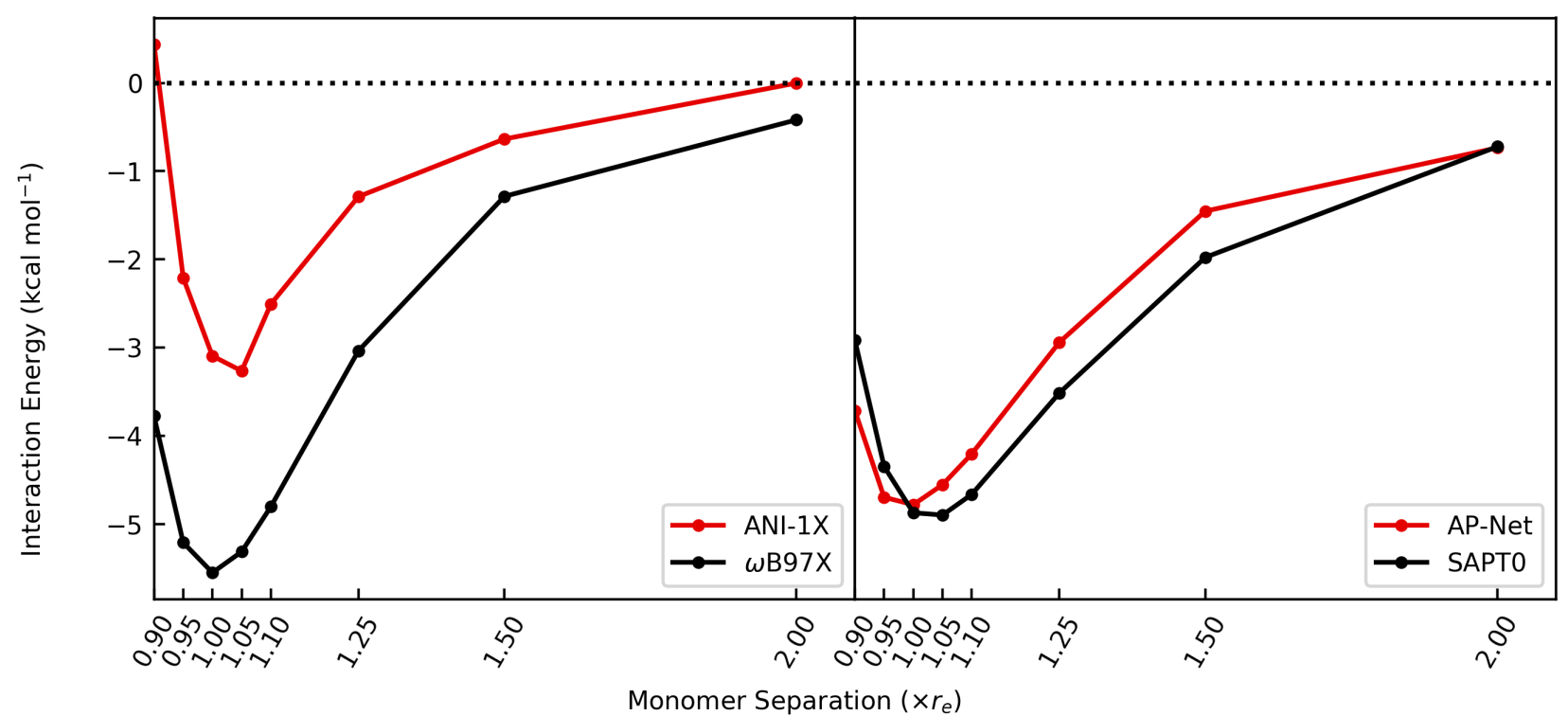

FIG. S58. ANI-1X and AP-Net are evaluated on the 8 geometries of the BenzenePeptideNHpi dimer in the S66x8 benchmark. Each ML model is compared to the level of theory used for parameterization: $\omega$ B97X/631G* for ANI-1X and SAPT0/jun-cc-pVDZ for AP-Net. 


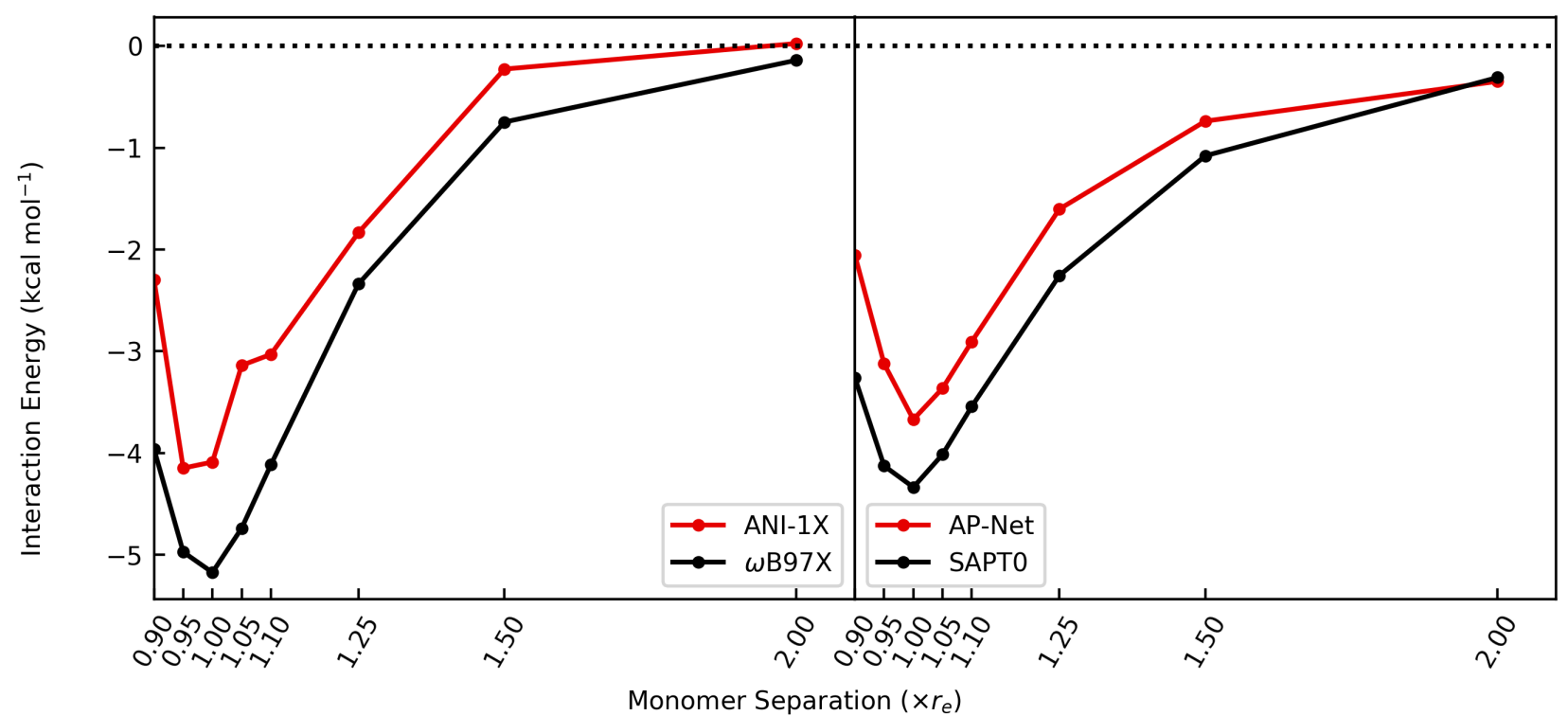

FIG. S59. ANI-1X and AP-Net are evaluated on the 8 geometries of the PyridinePyridineCHN dimer in the S66x8 benchmark. Each ML model is compared to the level of theory used for parameterization: $\omega \mathrm{B} 97 \mathrm{X} / 6-31 \mathrm{G}^{*}$ for ANI-1X and SAPT0/jun-cc-pVDZ for AP-Net.

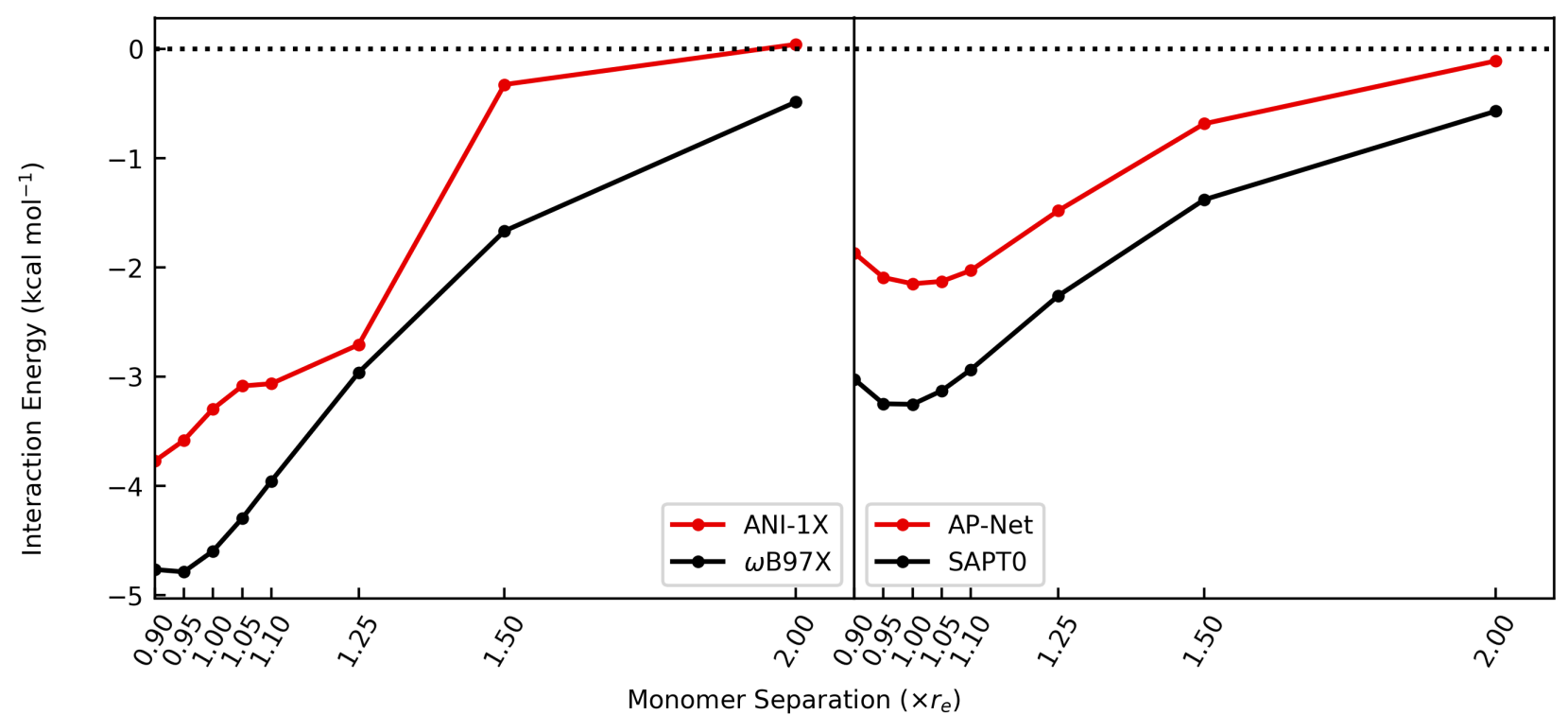

FIG. S60. ANI-1X and AP-Net are evaluated on the 8 geometries of the EthyneWaterCHO dimer in the S66x8 benchmark. Each ML model is compared to the level of theory used for parameterization: $\omega$ B97X/631G* for ANI-1X and SAPT0/jun-cc-pVDZ for AP-Net. 


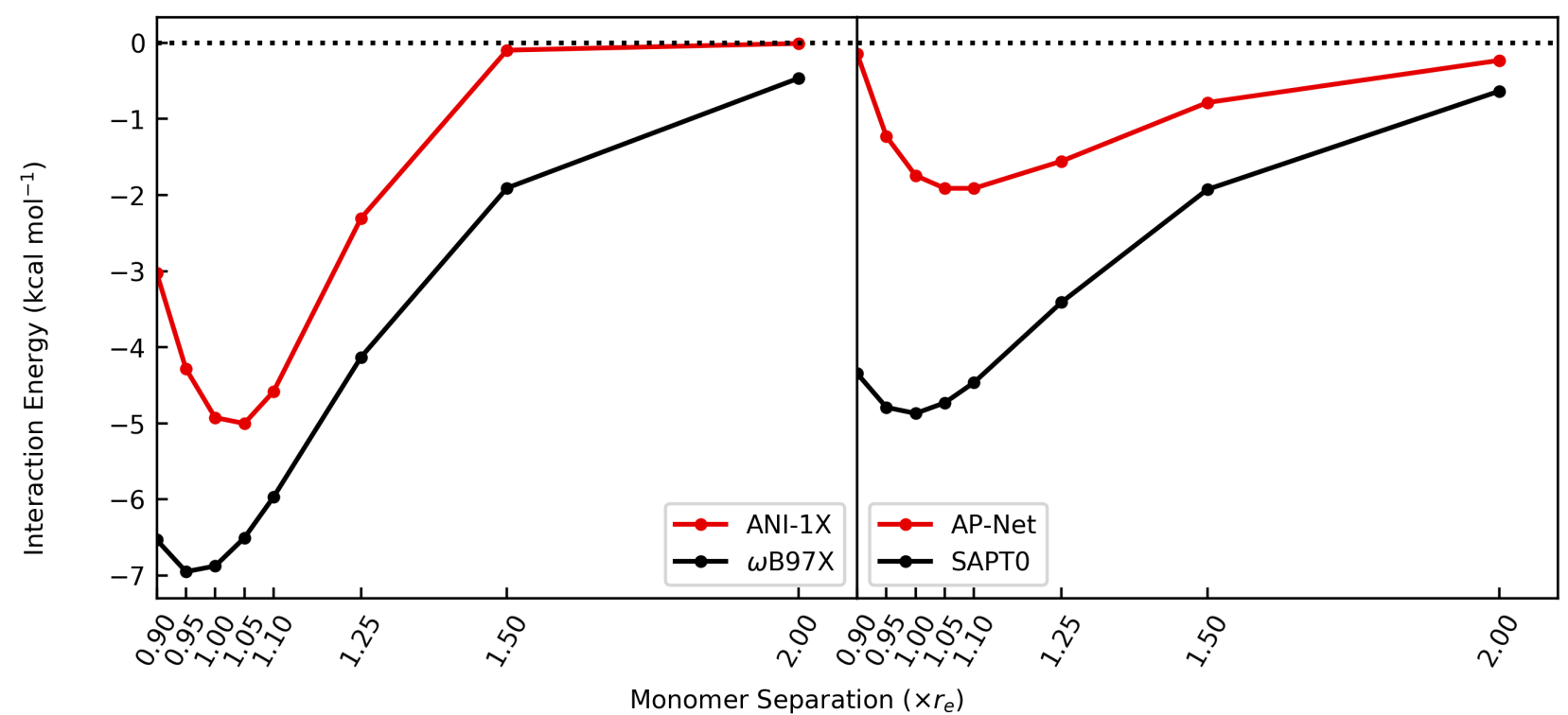

FIG. S61. ANI-1X and AP-Net are evaluated on the 8 geometries of the EthyneAcOHOHpi dimer in the S66x8 benchmark. Each ML model is compared to the level of theory used for parameterization: $\omega$ B97X/631G* for ANI-1X and SAPT0/jun-cc-pVDZ for AP-Net.

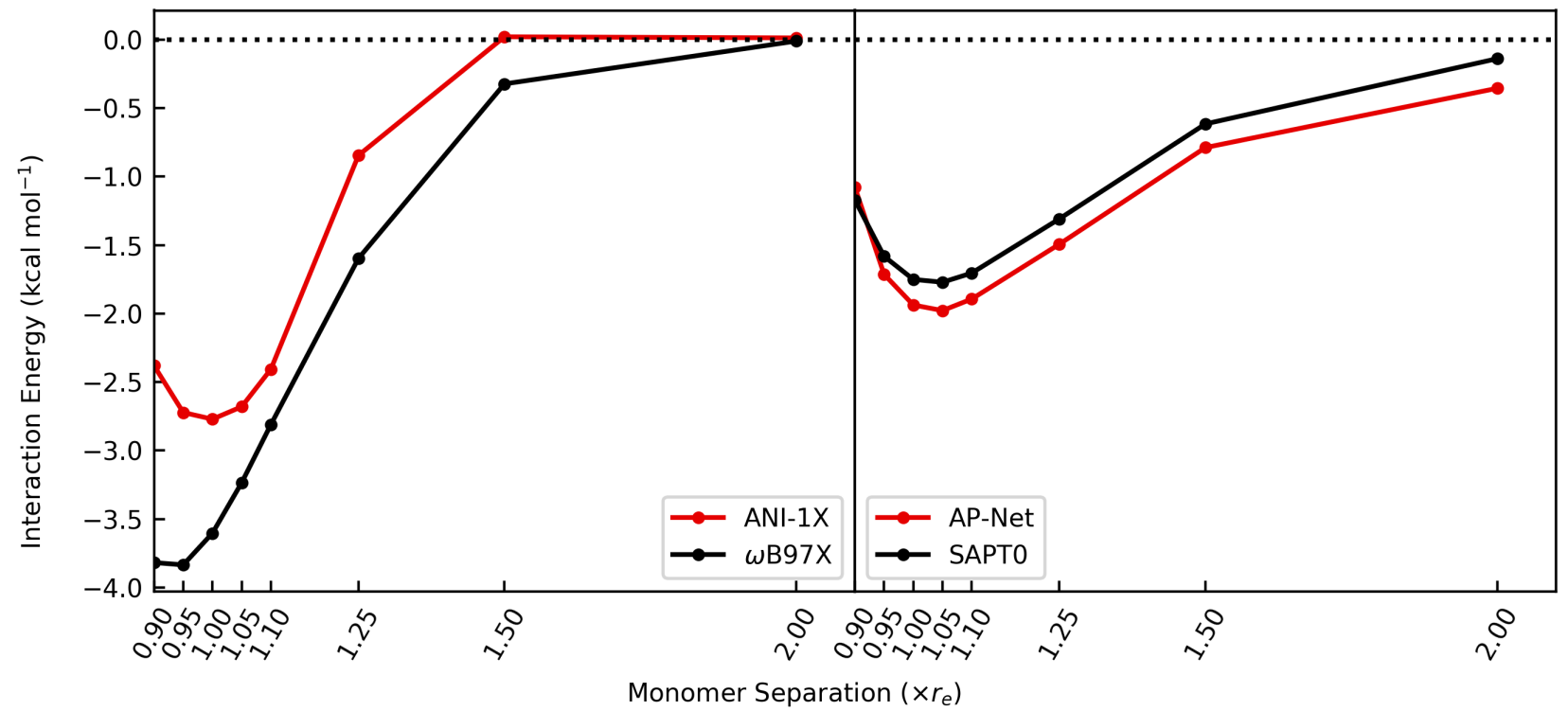

FIG. S62. ANI-1X and AP-Net are evaluated on the 8 geometries of the PentaneAcOH dimer in the S66x8 benchmark. Each ML model is compared to the level of theory used for parameterization: $\omega \mathrm{B} 97 \mathrm{X} / 6-31 \mathrm{G}^{*}$ for ANI-1X and SAPT0/jun-cc-pVDZ for AP-Net. 


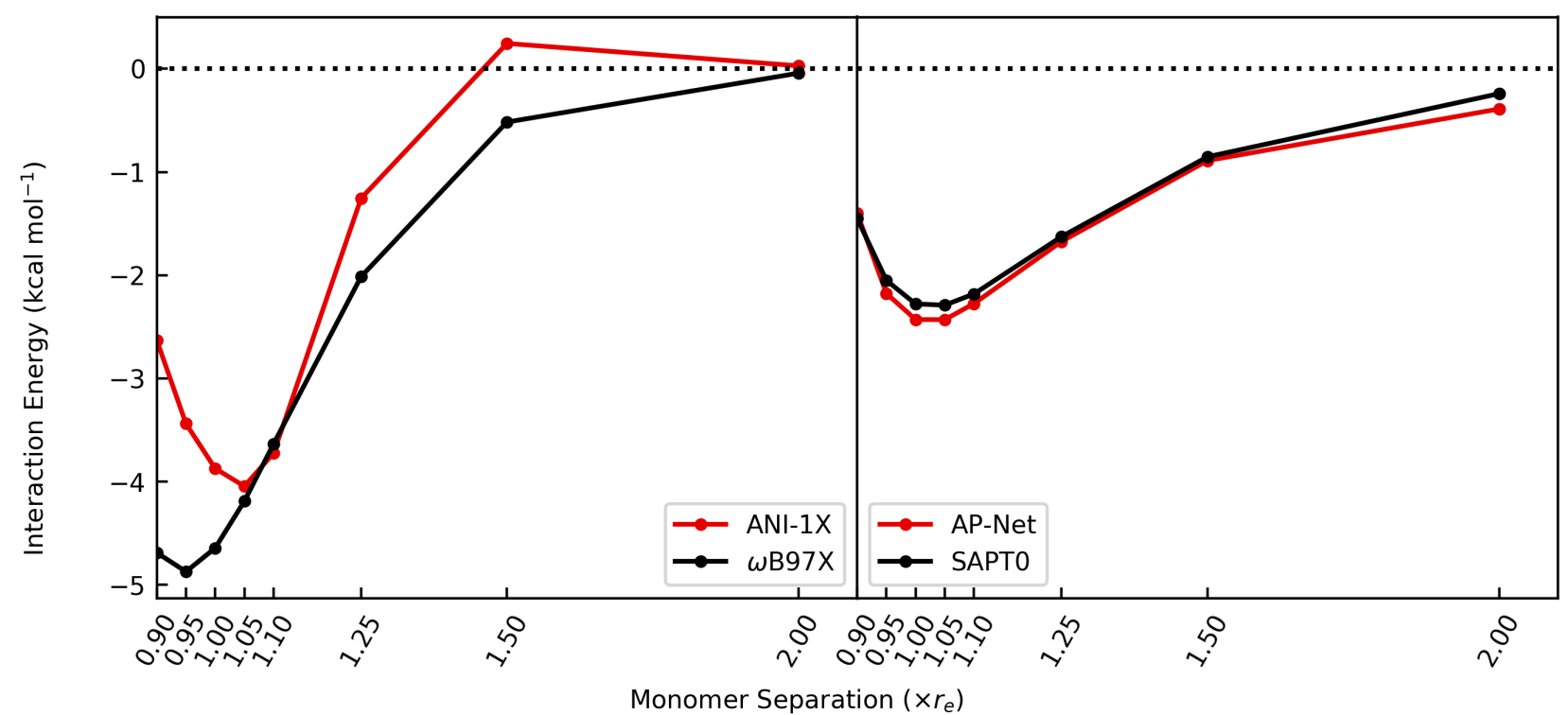

FIG. S63. ANI-1X and AP-Net are evaluated on the 8 geometries of the PentaneAcNH2 dimer in the S66x 8 benchmark. Each ML model is compared to the level of theory used for parameterization: $\omega \mathrm{B} 97 \mathrm{X} / 6-31 \mathrm{G}^{*}$ for ANI-1X and SAPT0/jun-cc-pVDZ for AP-Net.

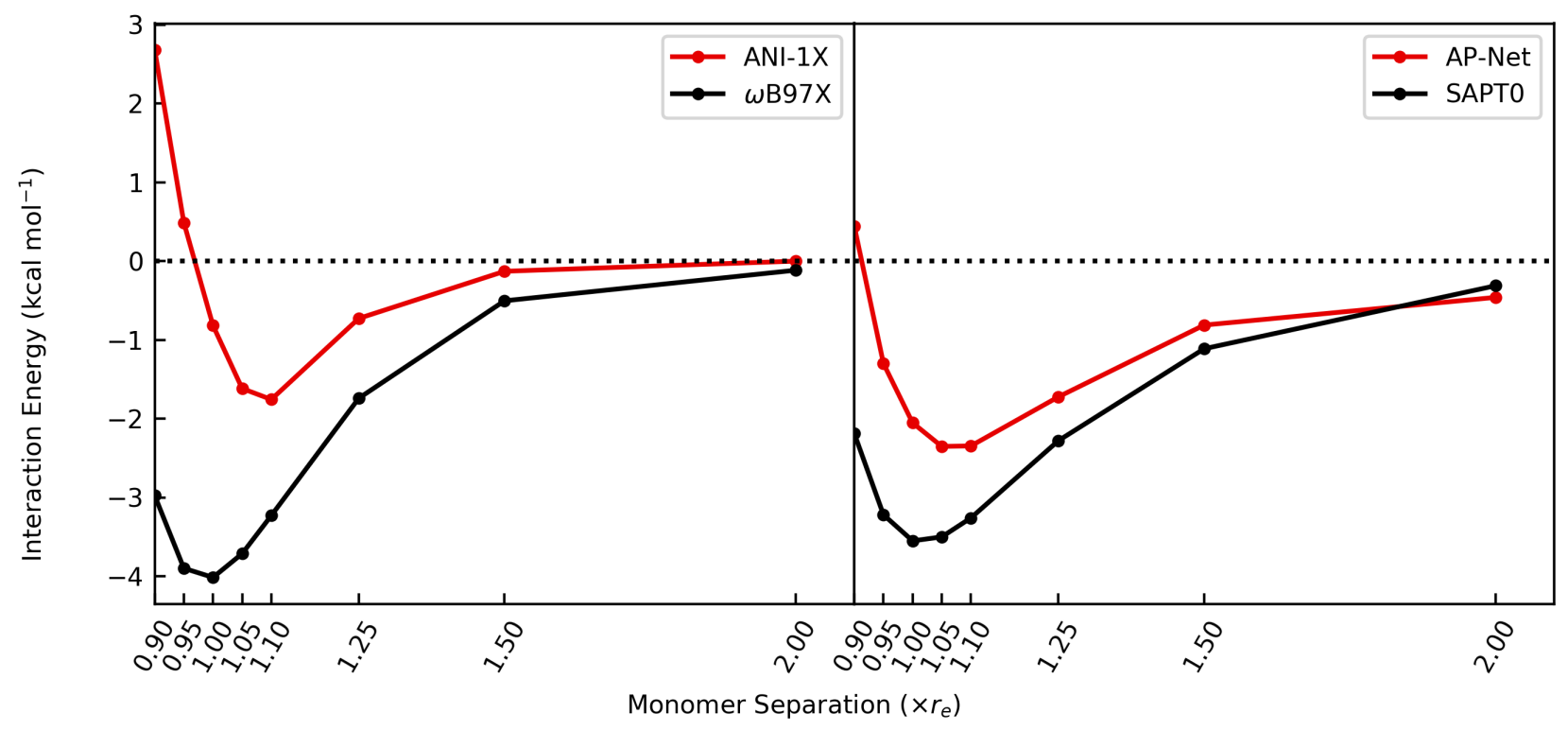

FIG. S64. ANI-1X and AP-Net are evaluated on the 8 geometries of the BenzeneAcOH dimer in the S66x8 benchmark. Each ML model is compared to the level of theory used for parameterization: $\omega \mathrm{B} 97 \mathrm{X} / 6-31 \mathrm{G}^{*}$ for ANI-1X and SAPT0/jun-cc-pVDZ for AP-Net. 


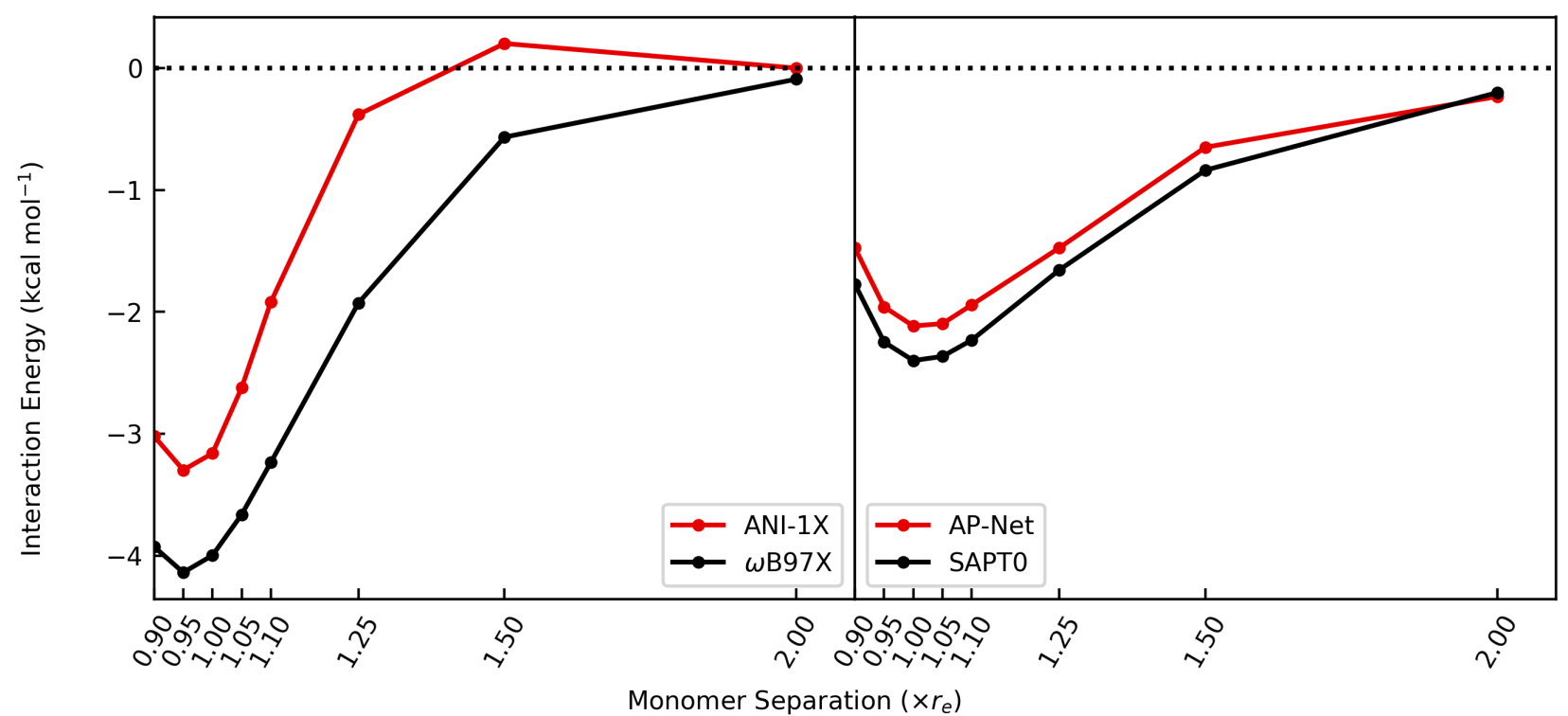

FIG. S65. ANI-1X and AP-Net are evaluated on the 8 geometries of the PeptideEthene dimer in the S66x8 benchmark. Each ML model is compared to the level of theory used for parameterization: $\omega \mathrm{B} 97 \mathrm{X} / 6-31 \mathrm{G}^{*}$ for ANI-1X and SAPT0/jun-cc-pVDZ for AP-Net.

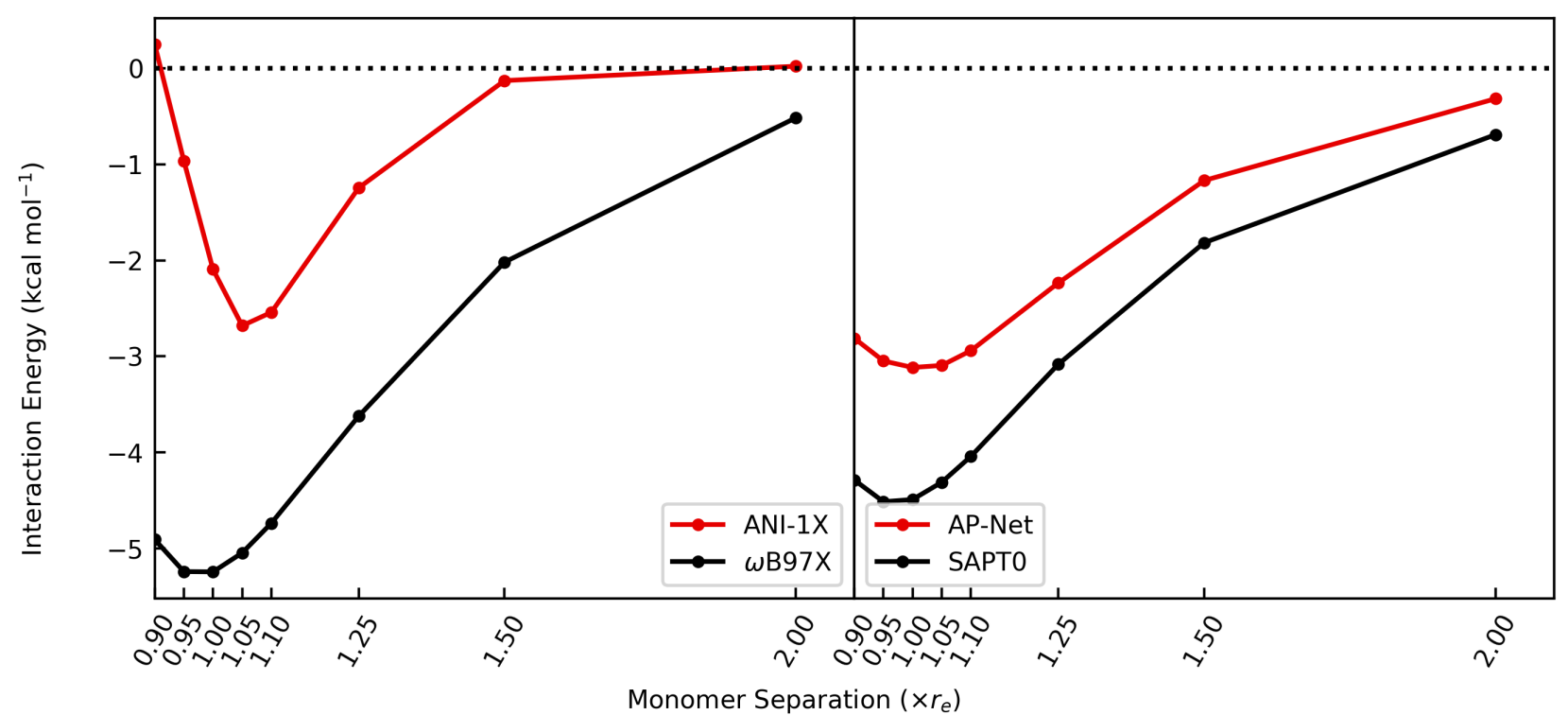

FIG. S66. ANI-1X and AP-Net are evaluated on the 8 geometries of the PyridineEthyne dimer in the S66x8 benchmark. Each ML model is compared to the level of theory used for parameterization: $\omega \mathrm{B} 97 \mathrm{X} / 6-31 \mathrm{G}^{*}$ for ANI-1X and SAPT0/jun-cc-pVDZ for AP-Net. 


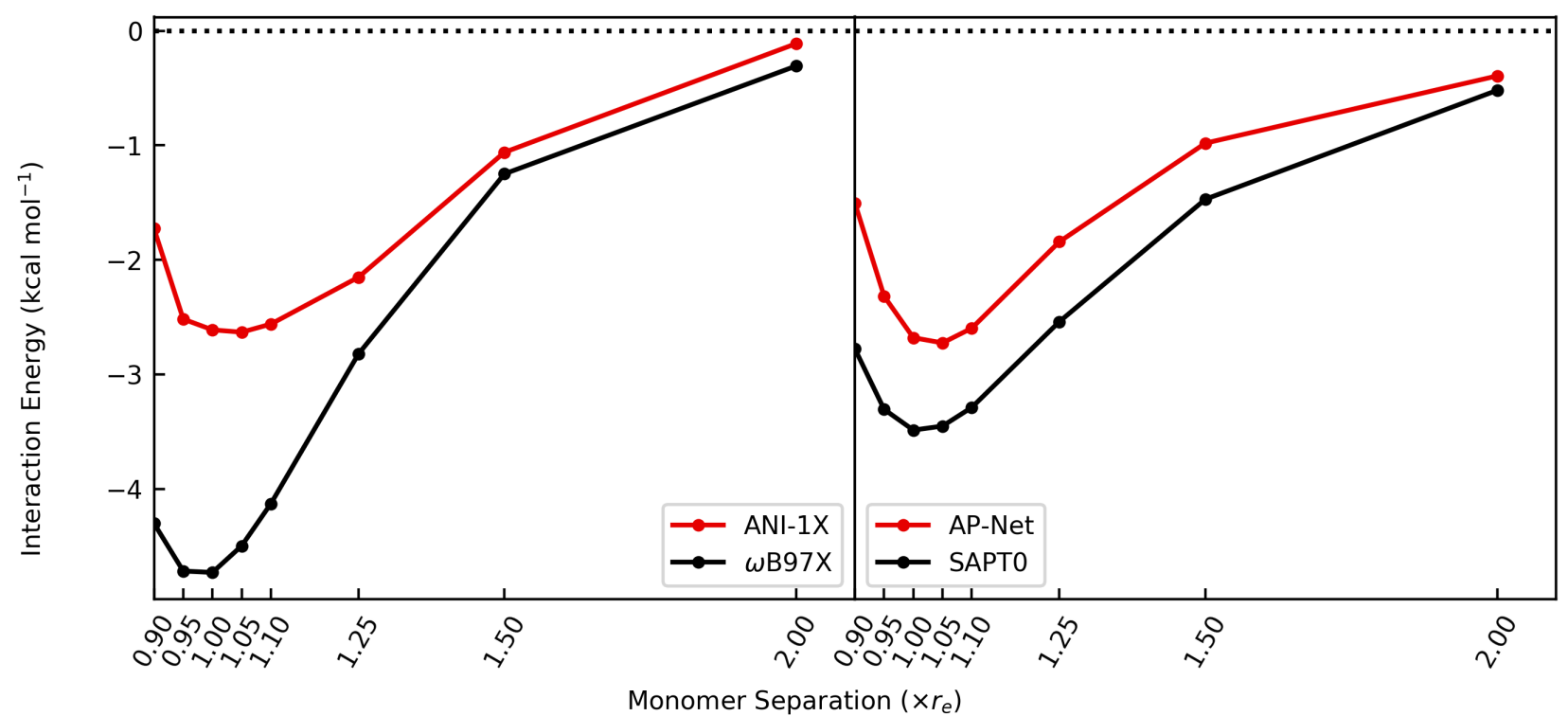

FIG. S67. ANI-1X and AP-Net are evaluated on the 8 geometries of the MeNH2Pyridine dimer in the S66x 8 benchmark. Each ML model is compared to the level of theory used for parameterization: $\omega \mathrm{B} 97 \mathrm{X} / 6-31 \mathrm{G}^{*}$ for ANI-1X and SAPT0/jun-cc-pVDZ for AP-Net. 
